\title{
THE SECOND CHINBURG CONJECTURE FOR QUATERNION FIELDS
}

\section{By}

\author{
MINH VAN TRAN, B.Math, M.Math
}

\author{
A Thesis \\ Submitted to the School of Graduate Studies \\ in Partial Fulfilment of the Requirements \\ for the Degree \\ Doctor of Philosophy \\ McMaster University \\ $\therefore$ \\ (C) Copyright by Minh Van Tran, 1996
}


THE SECOND CHINBURG CONJECTURE 
DOCTOR OF PIILOSOPIY (19!!6)

(Mathenatiss)
Mr.laster loniversity

Hamilum, Ontario

TITLE: The Second Chinburg Conjecture for Quaternion Fields.

AUTHOR: Minh Van 'Tran, B.Math, M.Math (University of Watcrloo)

SUPEIRVISOR: Professor V.P. Snaith

NUMBER OF PAGES: ix, 105 


\begin{abstract}
This thesis is a part of a program to study the Second Chinburg Conjecture. Let $N$ be a quaternion extension of the rationar; containing $Q\left(\sqrt{d_{1}}, \sqrt{d_{2}}\right)$, where $d_{1} \equiv 3$ $(\bmod 8)$ and $d_{2} \equiv 10(\bmod 16)$. A projective $\mathrm{Z}\left[Q_{8}\right]-\operatorname{module}$ inside the ring of integers $\mathcal{O}_{N}$ is constructed and is used, together with a cohomological classification of cohomologically trivial, 2-primary Q-modules, to compare $\Omega(N / Q, 2)$, Chinburg's second invariant, with $W_{N / Q}$, the root number class defined by $\mathrm{Ph}$. Cassou-Nougès and A. Fröhlich. The Second Chinburg Conjecture for this extension $N / Q$ is confirmed. Together with results of J. Hooper and S. Kim this calculation verifies the Second Chinburg Conjecture for all quaternion extensions of the rationals.
\end{abstract}

iii 


\section{Acknowledgements}

I would like to begin by thanking Dr. V. Snaith, my supervisor, not only for introducing me to the wonderful subject of Galois Module Structure and supervising this thesis, but also for allowing me to use some parts of his textbook. His understanding and thoughtfulness were invaluable throughout the period when this work was done.

I also would like to thank the other two members of my supervising commiltee, Dr. M. Kolster and Dr. C. Riehm for their helpful conversations.

A thank you is also extended to my friends, especially Jeff Hooper, Eric Derbez, and Spiro Daoussis.

For their continual support, my family deserves a special mention. The Department of Mathematics and Statistics deserves thanks for giving me this opportunity as does the Natural Science and Engineering Research Council of Canada. 


\section{Contents}

Preface vii

1 Introduction 1

1.1 Class Groups $\ldots \ldots \ldots \ldots \ldots \ldots \ldots \ldots \ldots$

1.2 Artin Root Numbers $\ldots \ldots \ldots \ldots \ldots \ldots$

1.3 Root Number Classes $\ldots \ldots \ldots \ldots \ldots \ldots \ldots$

1.4 Local Chinburg Invariants $\ldots \ldots \ldots \ldots \ldots \ldots \ldots$

1.5 Global Chinburg Invariants $\ldots \ldots \ldots \ldots \ldots \ldots \ldots \ldots$

2 Local Quaternion Extensions 28

2.1 The Two Extensions $\ldots \ldots \ldots \ldots \ldots \ldots \ldots \ldots \ldots$

2.2 The Fundamental 2-extension $\ldots \ldots \ldots \ldots \ldots \ldots \ldots$

2.3 The Injectivity of $k \ldots \ldots \ldots \ldots \ldots \ldots \ldots \ldots$ 
3 The Second Chinburg Conjecture

3.1 The Main Theorem $\ldots \ldots \ldots \ldots \ldots \ldots \ldots$. . . . .

3.2 Computation of $[M] \ldots \ldots \ldots \ldots \ldots \ldots$

3.3 Computation of $[X] \ldots \ldots \ldots \ldots \ldots \ldots$

3.4 Computation of $\left[\operatorname{Ker}\left(k^{\prime}\right)\right] \ldots \ldots \ldots \ldots \ldots \ldots$

Bibliography

100 


\section{Preface}

Let $K$ be an algebraic number field and $N / K$ be a finite normal extension with Galois group $G$. Now let $\mathcal{O}_{N}$ be the ring of algebraic integers of $N$. By Noether's theorem, $\mathcal{O}_{N}$ is projective as a $\mathrm{Z}[G]$-module if and only if $N / K$ is at most tamely ramified. In this case, Taylor proved that $\left[\mathcal{O}_{N}\right]=W_{N / K}$ in the class group $\mathcal{C L}(\mathrm{Z}[G])$ (Fröhlich's Conjecture), where $W_{N / K}$ is the root number class defined by CassouNoguès and Fröhlich by means of the Artin root numbers of the irreducible symplectic representations of $G$.

Chinburg defined Galois invariants $\Omega(N / K, i), i=1,2,3$ of $N / K$ in $\mathcal{C C}(\mathrm{Z}[G])$, proved $\Omega(N / K, 2)=\left[\mathcal{O}_{N}\right]$ for arbitrary extensions $N / K$ which are at most tamely ramified, and conjectured (The Second Chinburg Conjecture) that $\Omega(N / K, 2)=$ $W_{N / \kappa^{*}}$

This conjecture has a considerable amount of supporting evidence. In the first 
place it generalises the conjecture of Fröhlich. As to further evidence, several wildly ramified examples have been verified for $K=\mathrm{Q}$. The case where $G a l(N / \mathrm{Q}) \cong Q_{\mathrm{B}}$ and the prime 2 is not totally ramified was proved by Kim. The case where $N$ is a $p$-cyclotomic extension of degree $p^{n}$ over $Q$, for an odd regular prime $p$, was verified by Snaith. Recently, Greither has shown that this conjecture holds for all abelian extensions of $\mathbf{Q}$ with odd conductor. Apart from this direction, Ilolland proved that $\Omega(N / K, 2)-W_{N / K}$ is in the kernel group $D(\mathrm{Z}[G])$.

The purpose of this thesis is to study the Second Chinburg Conjecture for two families of quaternion fields over the rationals in which the prime 2 is totally ramified using the techniques of Snaith [32]. This work consists of three chapters.

Chapter 1 deals with definitions and basic propertics that lead to the statement of the Second Chinburg Conjecture. The main materials for this chapter can be found in Snaith [32, 33].

- In Chapter 2, two local quaternion extensions ( $Q_{8}$-extensions) of $Q_{2}$ are introduced. A special map $k$ is defined and proved to be injective. We also present some computational results using Maple which enable us to proceed with the third chapler.

Chapter 3 is devoted to verifying the Second Chinburg Conjecture for quaternion extensions over the rationals whose 2-adic completion is one of the $Q_{8}$-extensions of Section 2.1. Section 3.1 is concerned with the proof of the main result and the 
introduction of three $\mathrm{Z}\left[Q_{8}\right]$-modules used to compare $W_{N / \mathrm{Q}}$ with $\Omega(N / \mathrm{Q}, 2)$. The classes of these modules in $\mathcal{C L}\left(\mathrm{Z}\left[Q_{8}\right]\right)$ are computed in Sections 3.2 to 3.4 . 


\section{Chapter 1}

\section{Introduction}

The goal of this chapter is to present basic definitions and results which cnable us to state the Second Chinburg Conjecture and provide prerequisites. A more extensive reference for the material of this chapter are Fröhlich [11] and Snaith $[32,33]$.

\subsection{Class Groups}

In this section we briefly introduce Fröhlich's Hom-description of the class group of finitely generated, locally free modules.

Let $K$ be an algebraic number field, i.e. a finite extension of $Q$. Denote by $\mathcal{O}_{K}$ the ring of algebraic integers of $K$. A prime $\mathcal{P}$ of $K$ is an equivalence class 
of valuations of $K$. There are two types of primes: finite (non-Archimedean) and infinite (Archimedean). The finite primes belong to the prime ideals of $K$. The infinite primes correspond to the embeddings of $K$ into $\mathrm{R}$ or $\mathrm{C}$. We write $\mathcal{P} \chi \infty$ if $\mathcal{P}$ is finite and $\mathcal{P} \mid \infty$ if $\mathcal{P}$ is infinite. Each prime $\mathcal{P}$ determines a completion $K_{\mathcal{P}}$ of $K$. If $\mathcal{P} / \infty$, then $K_{\mathcal{P}}$ is a $\mathcal{P}$-adic number field. If $\mathcal{P} \mid \infty$, then $K_{\mathcal{P}}=\mathbf{R}$ or $K_{\mathcal{P}}=\mathbf{C}$.

The adele ring of $K$ is defined to be the ring given by the restricted product

$$
J(K)=\prod_{\mathcal{P} \text { prime }}^{*} K_{\mathcal{P}}
$$

where $\Pi^{\prime}$ signifies that we take those elements of the direct product $\Pi_{\mathcal{P}} K_{\mathcal{P}}$ for which almost all entries lie in the ring of integers $\mathcal{O}_{K_{\mathcal{P}}}$. When $\mathcal{P}$ is an infinite prime we adopt the convention that $\mathcal{O}_{K_{\mathcal{P}}}=K_{\mathcal{P}}$.

The group of ideles $j^{*}(K)$ is the group of units in $J(K)$

$$
J^{*}(K)=\left\{\left(x_{\mathcal{P}}\right) \in J(K) \mid x_{\mathcal{P}} \neq 0 \text { and almost everywhere } x_{P} \in \mathcal{O}_{K_{\mathcal{P}}}^{*}\right\}
$$

where, as usual, $\mathcal{O}_{K_{\mathcal{P}}}^{*}$ denotes the multiplicative group of units in $\mathcal{O}_{K_{\mathcal{P}}}$. The unit ideles is the subgroup of $J^{*}(K)$ in which every entry is a unit,

$$
U\left(\mathcal{O}_{K}\right)=\prod_{\mathcal{P} \text { prime }} \mathcal{O}_{K_{\mathcal{P}}}^{*}
$$

Now let $G$ be a finite group. We may extend the definitions of adèles and idèles 
to the group-rings, $\mathcal{O}_{K}[G]$ and $K[G]$. Define

$$
\begin{aligned}
J(K[G])= & \Pi_{\mathcal{P}}^{\prime} \text { prime } K_{P}[G] \\
J^{*}(K[G])= & \left\{\left(\alpha_{\mathcal{P}}\right) \in J(K[G]) \mid \alpha_{\mathcal{P}} \in \mathcal{O}_{K_{\mathcal{P}}}[G]^{*}\right. \text { for almost } \\
& \text { all } \left.\mathcal{P} \text { and } \alpha_{\mathcal{P}} \in K_{\mathcal{P}}[G]^{*} \text { otherwise }\right\} \\
U\left(\mathcal{O}_{K}[G]\right)= & \Pi_{\mathcal{P} \text { prime }} \mathcal{O}_{K_{\mathcal{P}}}[G]^{*}
\end{aligned}
$$

We now suppose that $E / K$ is a finite Galois extension with Galois group $G(E / K)$. In this case $G(E / K)$ acts on the set of primes of $E$ and hence acts upon the groups $J^{*}(E), U\left(\mathcal{O}_{E}\right), J^{*}(E[G])$ and $U\left(\mathcal{O}_{E}[G]\right)$. If $\mathcal{Q}$ is a prime of $E$ which divides the prime $\mathcal{P}$ of $K$, then $G\left(E_{\mathcal{Q}} / K_{\mathcal{P}}\right)$ is a subgroup of $G(E / K)$ which is called a decomposition group for $\mathcal{P}$ and depends only on $\mathcal{P}$, up to conjugation in $G(E / K)$.

Suppose now that $E$ is large enough to contain all $|G|$-th roots of unity. Denote by $R_{E}(G)$ the subring of $R(G)$ which is generated by representations of the form $\rho$ : $G \longrightarrow G L_{n}(E)$. In this case (see Snaith [1994a], Theorem 4.1.9(i)), $R_{E}(G)=R(G)$ and $G(E / K)$ acts upon $R(G)$ by the entry-by-entry action on a representation

$$
T: G \rightarrow G L_{n}(E)
$$

Therefore we may consider the group of $G(E / K)$-equivariant maps

$$
\operatorname{Hom}_{G(E / K)}\left(R(G), J^{*}(E)\right)=\left\{f: R(G) \rightarrow J^{*}(E) \mid f(g(z))=g(f(z))\right.
$$

for all $g \in G(E / K), z \in R(G)\}$. 
Example of such function can be found in Section 1.3.

More generally, if $L / K$ is a Galois extension which contains $E / K$ then $G(L / K)$ acts on $R(G)$ and $J^{*}(L)$, since $E / K$ is Galois and $G(E / K)$ is a quotient of $G(L / K)$. Therefore we may pass to the absolute Galois group $\Omega_{K}$ which is the topological group defined by

$$
\Omega_{K}=\lim _{\leftarrow} G(L / K)
$$

where $L \subset K^{c}$, a chosen algebraic closure of $K$, and the groups $G(L / K)$ are the Galois groups of finit.e Galois extensions $L$ of $K$. In this case we have

$$
\operatorname{Hom}_{\Omega_{K}}\left(R(G), J^{*}(E)\right)=H o m_{G(E / K)}\left(R(G), J^{*}(E)\right)
$$

Let $\mathrm{M}$ be an $\mathcal{O}_{K}[G]$-module of rank one which is locally free. This means that $M \otimes_{\mathcal{O}_{K}} \mathcal{O}_{K_{\mathcal{P}}}$ is a free $\mathcal{O}_{K_{\mathcal{P}}}[G]$-module on one generator $x_{\mathcal{P}}$ for each prime $\mathcal{P}$ of $K$ and that $M \otimes \mathcal{O}_{K} K$ is a free $K[G]$-module on one generator, $x_{0}$. Incidentally, a finitely generated module over $\mathrm{Z}[G]$ is locally free if it is, for example, a summand of a free module. Since $K[G]$ and $\mathcal{O}_{K_{\mathcal{P}}}[G]$ are subrings of $K_{\mathcal{P}}[G]$ we may compare these bases for the $K_{\mathcal{P}}[G]$-module $M \otimes_{\mathcal{O}_{K}} K_{\mathcal{P}}$. This means that there is a unit,

$$
\lambda_{\mathcal{P}} \in K_{\mathcal{P}}\left[G^{*}\right.
$$

which is defined by

$$
\lambda_{p} \cdot x_{0}=x_{\mathcal{p}} \in M \otimes \mathcal{O}_{K} K_{\mathcal{p}}
$$


In fact, $\lambda_{\mathcal{p}}$ will almost always lie in $\mathcal{O}_{K_{\mathcal{p}}}[G]^{*}$ so that we obtain an idèle

$$
\left(\lambda_{\mathcal{P}}\right) \in J^{*}(K[G])
$$

More generally, if $M$ is a locally free module of rank $n$ this comparison of bases will yield an invertible $n \times n$-matrix with adèlic entries

$$
\left(\lambda_{p}\right) \in G L_{n}(J(K[G]))
$$

If $R$ is a ring then we may define the first algebraic $K$-group, $K_{1}(R)$ as the abelianisation of $G L(R)=\cup G L_{n}(R)$ where $G L_{n}(R)$ is included into $G L_{n+1}(R)$ by sending a matrix, $X$ to the direct sum of $X$ with the $1 \times 1$ identity matrix. The commutator subgroup of $G L(R)$ is the subgroup $E(R)$ generated by elementary matrices so that $K_{1}(R)=G L(R) / E(R)$.

Hence we obtain

$$
\left(\lambda_{\mathcal{P}}\right) \in K_{1}(J(K[G]))=\prod_{\mathcal{P} \text { prime }} K_{1}\left(K_{\mathcal{P}}[G]\right)
$$

where the weak product indicates that almost all of the entries are in the first algabraic $K$-group $K_{\mathrm{t}}\left(\mathcal{O}_{K_{p}}[G]\right)$.

Now suppose that $T$ is a representation

$$
T: G \rightarrow G L_{m}(E)
$$


We may apply $T$ to each $\lambda_{\mathcal{P}} \in G L_{n}\left(K_{\mathcal{P}}[G]\right)$ (or $\lambda_{\mathcal{P}} \in K_{1}\left(K_{\mathcal{P}}[G]\right)$ ) to obtain an invertible matrix

$$
T\left(\lambda_{\mathcal{P}}\right) \in M_{m n}\left(K_{\mathcal{P}} \otimes_{K} E\right)
$$

where $M_{n}(A)$ denotes the $n \times n$ matrices with entries in $A$ (or $T\left(\lambda_{\mathcal{P}}\right) \in K_{1}\left(K_{\mathcal{P}} \otimes_{K}\right.$ $\left.E) \cong\left(K_{\mathcal{P}} \otimes_{K^{*}} E\right)^{*}\right)$. There is a ring isomorphism of the form

$$
K_{\mathcal{P}} \otimes_{K} E \cong \prod_{\substack{\mathbf{Q} \mid \mathcal{P} \\ \mathcal{Q} \text { prime of } E}} E_{\mathcal{Q}}
$$

Therefore we obtain an element

$$
\operatorname{det}\left(T\left(\lambda_{\mathcal{P}}\right)\right) \in \prod_{\substack{\text { e prime of } E \\ \text { e } E}} E_{\mathcal{Q}}^{*}
$$

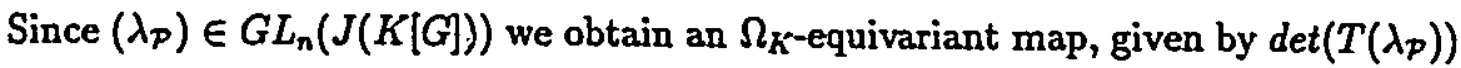
at the primes of $E$ which divide $\mathcal{P}$,

$$
\operatorname{Det}\left(\left(\lambda_{\mathcal{P}}\right)\right) \in \operatorname{Hom}_{\Omega_{K}}\left(R(G), J^{*}(E)\right)
$$

Now let us consider the dependence of this construction upon the choices of the basis elements, $x_{0}$ and $x_{\mathcal{P}}$. The discussion is similar in the case of modules of rank greater than one. If we replace $x_{\mathcal{P}}$ by another generator, $x_{\mathcal{P}}^{\prime}$, these choices will be related by an equation

$$
x_{\mathcal{P}}^{\prime}=u_{\mathcal{P}} x_{\mathcal{P}}
$$


for some $u_{\mathcal{P}} \in \mathcal{O}_{K_{\mathcal{P}}}[G]^{*}$ so that we obtain a unit idèle

$$
u=\left(u_{\mathcal{P}}\right) \in U\left(\mathcal{O}_{K}[G]\right)
$$

and $\operatorname{Det}\left(\left(\lambda_{\mathcal{P}}\right)\right)$ will be altered by multiplication by

$$
\operatorname{Det}(u) \in \operatorname{Det}\left(U\left(\mathcal{O}_{K^{K}}[G]\right)\right) \subset \operatorname{Hom}_{\Omega_{K}}\left(R(G), J^{*}(E)\right)
$$

Also, there is a diagonal embedding of $E^{*}$ into $J^{*}(E)$ which induces an inclusion

$$
H o m_{\Omega_{K}}\left(R(G), E^{*}\right) \subset H_{\Omega_{K^{*}}}\left(R(G), J^{*}(E)\right) \text {. }
$$

By a similar argument, changing $x_{0}$ to $x_{0}^{\prime}$ will change $\operatorname{Det}\left(\left(\lambda_{\mathcal{P}}\right)\right)$ by a function which lies in the subgroup $\operatorname{Horn}_{\Omega_{K}}\left(R(G), E^{*}\right)$. Therefore we have associated to each finitely generated, locally free $\mathcal{O}_{K}[G]$-module, $M$, a well-defined element

$$
\operatorname{Det}[M] \in \frac{\operatorname{Hom}_{\Omega_{K}}\left(R(G), J^{*}(E)\right)}{\operatorname{Hom}_{\Omega_{K}}\left(R(G), E^{*}\right) \cdot \operatorname{Det}\left(U\left(\mathcal{O}_{K}[G]\right)\right)} .
$$

Now let us recall the definition of the class-group, $\operatorname{CL}\left(\mathcal{O}_{K}[G]\right)$. This is defined to be the Grothendieck group of finitely generated, locally free $\mathcal{O}_{K}[G]$-modules. To be precise, consider the set of isomorphism classes of finitely generated, locally free $\mathcal{O}_{K}[G]$-modules, Mod.l.f. $\left(\mathcal{O}_{K}[G]\right)$. This set is a monoid if we endow it with an addition operation defined by

$$
[M]+[N]=[M \oplus N]
$$


where $[-]$ denotes an isomorphism class. We now define an equivalence relation on Mod.l.f. $\left(\mathcal{O}_{K}[G]\right)$ which is generated by two types of relations:

(i) $[M] \sim[N]$ if for some $m, n$,

$$
M \oplus\left(\mathcal{O}_{K}[G]\right)^{m} \cong N \oplus\left(\mathcal{O}_{K}[G]\right)^{n}
$$

and

(ii) $[A]+[C] \sim[B] \quad$ if there exists an exact sequence of $\mathcal{O}_{K}[G]$-modules

$$
0 \longrightarrow A \longrightarrow B \longrightarrow C \longrightarrow 0
$$

Definition 1.1.1 With the above notation,

$$
\mathcal{C L}\left(\mathcal{O}_{K}[G]\right)=\left\{\text { Mod.l.f. }\left(\mathcal{O}_{K}[G]\right)\right\} / \sim
$$

Incidentally, it is known that $\mathcal{C L}\left(\mathcal{O}_{K}[G]\right)$ is generated by locally free $\mathcal{O}_{K}[G]$-modules of rank one. The following connection between the Det-construction and the classgroup is called the 'Hom-description' and is due to Fröhlich.

Theorem 1.1.2 (Curtis and Reiner [7], page 334; Fröhlich [9]) With the notation introduced above there is an isomorphism

$$
\operatorname{Det}: \mathcal{C L}\left(\mathcal{O}_{K}[G]\right) \stackrel{\cong}{\longrightarrow} \frac{H o m_{\Omega_{K}}\left(R(G), J^{*}(E)\right)}{\operatorname{Hom}_{\Omega_{K}}\left(R(G), E^{*}\right) \cdot \operatorname{Det}\left(U\left(\mathcal{O}_{K}[G]\right)\right)}
$$

which sends a locally free module, $M$, to the class of $\operatorname{Det}\left(\left(\lambda_{p}\right)\right)$ defined in Section 1.1. 


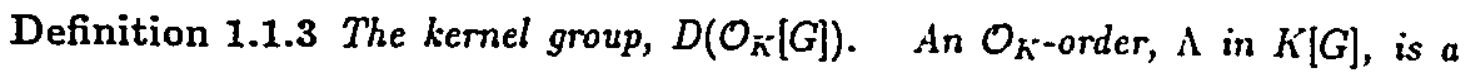
subring containing $\mathcal{O}_{K}$, which is a finitely generated, projective $\mathcal{O}_{K^{-}}$-module such that

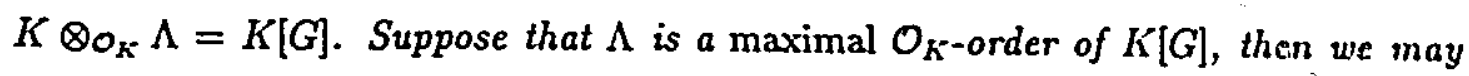
define the kernel group, $D\left(\mathcal{O}_{K}[G]\right)$ by

$$
D\left(\mathcal{O}_{K}[G]\right)=\operatorname{ker}\left(\mathcal{C L}\left(\mathcal{O}_{K}[G]\right) \longrightarrow \mathcal{C L}(\Lambda)\right)
$$

where $\mathcal{C L}(\Lambda)$ is the class-group of $\Lambda$, defined by a Grothendieck group construction analogous to that of Definition 1.1.1. The group, $D\left(\mathcal{O}_{K}[G]\right)$, defined in this manner, is independent of the choice of $\Lambda$.

There is a Hom-description of this group also.

To describe this we need to introduce a subgroup

$$
H o m_{\Omega_{K}}^{+}\left(R(G), \mathcal{O}_{E}^{*}\right) \subset H o m_{\Omega_{k}}\left(R(G), \mathcal{O}_{E}^{*}\right)
$$

A complex representation $T$ is symplectic if it is of the form

$$
T: G \longrightarrow G L_{n}(\mathbf{H}) \stackrel{c}{\longrightarrow} G L_{2 n}(\mathbf{C}),
$$

where $\mathbf{H}=\mathbf{R}[i, j, k]$ is the division ring of real quaternions and $c$ is the complexification homomorphism, i.e. if we write $X=\dot{Y}+W j$, where $Y, W \in G L_{\mathrm{n}}(\mathbf{R}[i])$, 
then

$$
c(X)=\left(\begin{array}{cc}
Y & -W \\
\bar{W} & \bar{Y}
\end{array}\right) .
$$

A character $\chi$ is called symplectic if it is the character of a symplectic representation. Suppose that $\chi$ is a symplectic character. Then complex conjugation fixes $\chi$ in $R(G)$ so that if $f \in \operatorname{Hom}_{\Omega_{K}}\left(R(G), E^{*}\right)$ and if $K$ is a subfield of the real numbers $\mathbf{R}$, then $f(\chi)$ lies in $\mathbf{R}$ for every Archimedean prime of $E$ which extends $K \subset \mathbf{R}$. Therefore it makes sense to define $H_{o m_{\Omega_{K}}^{+}}^{+}\left(R(G), E^{*}\right)$ to be

$$
\left\{f \in H o m_{\Omega_{K}}\left(R(G), E^{*}\right) \mid f(\chi) \text { is positive, } \chi \text { symplectic }\right\}
$$

where positive means that $f(\chi)$ is positive under every Archimedean place of $E$ which lies over a real place of $K$.

Similarly we may define

$$
H o m_{\Omega_{K}}^{+}\left(R(G), \mathcal{O}_{E}^{*}\right)=\left\{f \in H o m_{\Omega_{K}}^{+}\left(R(G), E^{*}\right) \mid i m(f) \subset \mathcal{O}_{E}^{*}\right\}
$$

Theorem 1.1.4 (Curtis and Reiner [7], page 334) The isomorphism of Theorem 1.1.9 induces an isomorphism

$$
\text { Det : } D\left(\mathcal{O}_{K}[G]\right) \stackrel{\Xi}{\longrightarrow} \frac{\operatorname{Hom}_{\Omega_{K}}\left(R(G), U\left(\mathcal{O}_{E}\right)\right)}{\operatorname{Hom}_{\Omega_{K}}^{+}\left(R(G), \mathcal{O}_{E}^{+}\right) \cdot \operatorname{Det}\left(U\left(\mathcal{O}_{K}[G]\right)\right)}
$$


Remark 1.1.5 $\mathcal{O}_{E_{\mathbb{Q}}}[G]$ is a maximal $\mathcal{O}_{E_{\mathcal{Q}}}$-order in $E_{\mathcal{Q}}[G]$ for all primes, $\mathcal{Q}$, which do not divide the order of $G$. Using this fact one may rewrite Theorem 1.1.4 as an isomorphism of the following form.

$$
D e t: D\left(\mathcal{O}_{K}[G]\right) \stackrel{\cong}{\longrightarrow} \frac{H o m_{\Omega_{K}}\left(R(G), \prod_{\mathcal{C}|G|} \mathcal{O}_{E_{\varepsilon}}\right)}{H o m_{\Omega_{K}}^{+}\left(R(G), \mathcal{O}_{E}^{-}\right) \cdot \operatorname{Det}\left(\prod_{\mathcal{P}|| G \mid} \mathcal{O}_{K_{\mathcal{P}}}[G]^{\bullet}\right)}
$$

Remark 1.1.6 Let $Q_{8}$ be a quaternion group of order 8, i.e.

$$
Q_{8}=\left\{x, y \mid x^{2}=y^{2}, y^{4}=1, x y x^{-1}=y^{-1}\right\}
$$

then $\mathcal{C L}\left(\mathrm{Z}\left[Q_{8}\right]\right)$ has order 2 (Martinet [25]). A new definition of the isomorphism of the form

$$
n: \mathcal{C L}\left(\mathrm{Z}\left[Q_{8}\right]\right) \rightarrow(\mathrm{Z} / 4)^{*} \cong \mathrm{Z} / 2
$$

can be found in Snaith [33], Section 5.2.

\subsection{Artin Root Numbers}

Let $L / K$ be a finite, Galois extension of number fields and let $\chi$ be the character of a finite dimensional complex representation of $G(L / K)$. The extended Artin $L$ function, $\Lambda_{K}(s, \chi)$ (see Snaith [33], page 334), is a meromorphic function of the complex variable, $s$, satisfying the following properties: 
(i) $\Lambda_{K}\left(s, \chi_{1} \oplus \chi_{2}\right)=\Lambda_{K}\left(s, \chi_{1}\right) \Lambda_{K}\left(s, \chi_{2}\right)$;

(ii) If $K \subset L \subset N$ is a chain of finite Galois extensions and $G(N / K) \rightarrow G(L / K)$ is the canonical map, then

$$
\Lambda_{K}\left(s, \operatorname{In} f_{G(L / K)}^{G(N / K)}(\chi)\right)=\Lambda_{K}(s, \chi)
$$

(iii) If $F$ is an intermediate field of $L / K$ and $\psi$ is a character of $G(L / F)$, then

$$
\Lambda_{K}\{s, \operatorname{Ind} G(L / F)(\psi))=\Lambda_{F}(s, \psi) ;
$$

(iv) If $\bar{\chi}$ denotes the complex conjugation of $\chi$, then

$$
\Lambda_{K}(1-s, \chi)=W_{K}(\chi) \Lambda_{K}(s, \bar{\chi})
$$

where $W_{K}(\chi)$ is a complex number of absolute value one.

The invariant $W_{K}(\chi)$ is called the Artin root number of $\chi$. It satisfies the following properties:

(i) $W_{K}\left(\chi_{1} \oplus \chi_{2}\right)=W_{K}\left(\chi_{1}\right) W_{K}\left(\chi_{2}\right)$;

(ii) If $K \subset L \subset N$ is a chain of finite Galois extensions and $G(N / K) \rightarrow G(L / K)$ is the canonical map, then

$$
W_{K}\left(\operatorname{In} f_{G(L / K)}^{G(N / K)}(\chi)\right)=W_{K}(\chi)
$$

(iii) If $F$ is an intermediate field of $L / K$ and $\psi$ is a character of $G(L / F)$, then

$$
W_{K}(\operatorname{Ind} G(L / F)(\psi))=W_{F}(\psi)
$$


The Artin root number $W_{K}(\chi)$ may be determined locally in this casc. If $v$ is a prime of $K$ and $w$ is a prime of $L$ above $v$ with the coriesponding decomposition group $G\left(L_{w} / K_{v}\right)$, let $\chi_{v}=\operatorname{Res}_{G\left(L_{w} / K_{v}\right)}^{G(L / K)}(\chi)$ and $W_{v}=W_{K_{v}}$. Then by a result of Tate in 1950, (see Snaith [32], Theorem 1.4.11),

$$
W_{K}(x)=\prod_{v} W_{v}\left(x_{v}\right)
$$

In the above formula, the term $W_{v}\left(\chi_{v}\right)$ is called the local root number associated to $\chi$. To give the definition of the local root number, we first define the abelian local Gauss sum.

Let $K / Q_{p}$ be an extension of local fields. We define the non-trivial additive character $\psi_{K}: K \rightarrow \mathrm{C}^{*}$ as the composition of the following four maps:

$$
K \stackrel{\operatorname{Tr}_{K / Q_{p}}}{\longrightarrow} Q_{p} \stackrel{\lambda}{\longrightarrow} Q_{p} / Z_{p} \stackrel{\mu}{\longrightarrow} Q / Z \stackrel{\text { exp }}{\longrightarrow} C^{*}
$$

where:

(i) $\operatorname{Tr}_{K / Q_{p}}$ is the trace homomorphism,

(ii) $\lambda$ is the canonical projection,

(iii) $\mu$ is the canonical injection which maps $Q_{p} / Z_{p}$ onto the $p$-component of the divisible group $\mathbf{Q} / \mathbf{Z}$,

(iv) exp is the exponential map $x \mapsto e^{2 \pi i x}$. 
For every $x \in Q_{p}$, there is a rational number $r$, uniquely defined modulo 1 , such that $x-r \in \mathbf{Z}_{p}$. Then $\exp \circ \mu \circ \lambda(x)=\exp \circ \mu \circ \lambda(r)=e^{2 \pi i r}$.

The codifferent of $K, \mathcal{D}_{K}^{-1}$, is defined to be the largest fractional ideal of $K$ for which $\psi_{K}$ is trivial.

Now suppose that $L / K$ is a Galois extension of local fields and that $\theta$ is the character of a one-dimensional Galois representation. We may factor $\theta$ uniquely through the abelianisation $G(L / K)^{\text {ab }}$. From local class field theory, there is a natural isomorphism of the form

$$
\quad G(L / K)^{a b} \stackrel{\cong}{\longrightarrow} K^{*} / N_{L / K}\left(L^{*}\right),
$$

and hence we may identify $\theta$ with a unique continuous homomorphism of the form

$$
0: K^{*} \longrightarrow C^{*}
$$

which is trivial on $N_{L / K}\left(L^{*}\right)$.

Making this identification, we define the associated abelian local Gauss sum $\tau(\theta)$ by the formula

$$
\tau(\theta)=\sum_{x \in U_{K}^{0} / U_{K}^{(0)}} \theta(x / c) \psi_{K}(x / c)
$$

where $n(\theta)$ is the least integer such that the character $\theta$ is trivial on the subgroup $U_{K}^{n(\theta)}$ and $c$ is a generator of the ideal $f(\theta) \mathcal{D}_{K}$. Here, $f(\theta)=\mathcal{P}_{K}^{n(\theta)}$ is called the local Artin conductor ideal of $\theta$ and $\mathcal{D}_{K}$ is the different of $K$. 
The local root number of the character $\theta$ of a one-dimensional Galois representation of a local field $K$ can be defined explicitly in the foliowing manner.

(i) If $K=\mathrm{C}$, set $W_{K}(\theta)=1$.

(ii) If $K=\mathbf{R}$, set

$$
W_{K}(\theta)=\left\{\begin{array}{cc}
1 & \text { if } \theta \text { is trivial } \\
-i & \text { otherwise }
\end{array}\right.
$$

(iii) If $K$ is non-Archimedean, set

$$
W_{K}(\theta)=\tau(\bar{\theta}) /(\sqrt{N(f(\theta))})
$$

Now let $L / K$ be a Galois extension of local ficlds. By a result of Tate [37], the abelian root number defined above can be extended uniquely to the local root number homomorphism

$$
W_{K}: R(G(L / K)) \rightarrow \mathrm{C}^{*}
$$

which takes values in complex numbers of norm one and satisfies the following properties:

(i) $W_{K}\left(\chi_{1} \oplus \chi_{2}\right)=W_{K}\left(\chi_{1}\right) W_{K}\left(\chi_{2}\right)$;

(ii) If $K \subset L \subset N$ is a chain of finite Galois extensions and $G(N / K) \rightarrow G(L / K)$ is the canonical map, then

$$
W_{K}\left(\operatorname{In} f_{G(L / K)}^{G(N / K)}(\chi)\right)=W_{K}(\chi)
$$


(iii) If $F$ is an intermediate field of $L / K$ and $\chi$ is a character of $G(L / F)$, then

$$
W_{K}\left(\operatorname{In} d_{G(L / F)}^{G(L / K)}(\chi-\operatorname{dim}(\chi))\right)=W_{F}(\chi)
$$

Note that $W_{K}(1)=1$.

The non-abelian local Gauss sum is defined from the root number by the formula

$$
W_{h}(\chi)=\tau(\bar{\chi}) /(\sqrt{N(f(\chi))})
$$

\subsection{Root Number Classes}

In this section we shall describe the root number class which was introduced $b y$ Cassou-Noguès and Fröhlich (see Snaith [33], Chapter 7).

Let $L / K$ be a finite; Galois extension of number fields and let $\chi$ be the character of a finite-dimensional complex representation of $G(L / K)$. To this data, in Section 1.2, is attached the Artin root number of $\chi$. If $\chi$ is a symplectic character, then it follows from the functional equation of the extended Artin $L$-function that $W_{K}(\bar{\chi})=\overline{W_{K}(\chi)}$. Hence if $\chi=\bar{\chi}$, then $W_{K}(\chi)$ is real; as it is of absolute value 1 it must be \pm 1 . Therefore, if $\chi$ is symplectic, then $W_{K}(\chi) \in\{ \pm 1\}$.

In terms of these invariants of analytic origins we may define a class

$$
W_{L / K} \in \mathcal{C L}(\mathbf{Z}[G(L / K)])
$$


in the following manner. We shall use the Hom-description of Theorem 1.1. Let $E / \mathrm{Q}$ be a large Galois extension of the rational numbers, as in Section 1.1.

The absolute Galois group $\Omega_{Q}$ acts transitively on the infinite places of $E$. Let $v_{\infty}$ denote one of these places. Define a homomorphism

$$
W_{L / K}^{\prime} \in H o m_{\Omega_{q}}\left(R(G(L / K)), J^{*}(E)\right)
$$

by the following formula for the $v$-th coordinate of $W_{L / K}^{\prime}(x)$, where $\chi$ is any irreducible character:

$$
W_{L / K}^{\prime}(\chi)_{v}= \begin{cases}1 & \text { if } v \text { is finite } \\ 1 & \text { if } \chi \text { is not symplectic } \\ \alpha^{-1}\left(W_{K}(\alpha(\chi))\right) & \text { if } \chi \text { symplectic, } v=\alpha^{-1}\left(v_{\infty}\right), \alpha \in \Omega_{Q} .\end{cases}
$$

By construction, $W_{L / K}^{\prime}(\chi)$ is $\Omega_{Q}$-equivariant and therefore represents a class, $W_{L / K}$, in the class-group. However, we must show that the construction of $W_{L / K}$ is independent of the choice of $v_{\infty}$. In the tamely ramified case it is possible to define $W_{L / K}$ without making a choice of $v_{\infty}$ and in this form the construction of $W_{L / K}$ was introduced by $\mathrm{Ph}$. Cassou-Noguès [1]. The definition of $W_{L / K}$ for wildly ramified extensions $L / K$ is due to A. Fröhlich [10] (see Chinburg [3], page 18).

Proposition 1.3.1 The class, $W_{L / K}$, defined above is independent of the choice of 
$v_{\infty}$ and satisfies

$$
2 W_{L / K}=0 \in \mathcal{C L}(\mathrm{Z}[G(L / K)])
$$

Proof The fact that $W_{L / K}$ is 2-torsion follows from the fact that Artin root numbers of symplectic characters lie in $\{ \pm 1\}$.

If $\chi$ is a character of $G(L / K)$, let $N(f(\chi))$ denote the absolute norm of the Artin conductor ideal of Section 1.2. Write $\sqrt{N(f(\chi))}$ for the positive square-root of $N(f(\chi))$. Since $\sqrt{N(f(\chi))}$ is a positive real number, the elements

$$
\alpha^{-1}(\sqrt{(N f(\alpha(\chi)))})=\alpha^{-1}(\sqrt{(N f(\chi))}) \in E_{\alpha-1}^{*}\left(v_{\infty}\right)
$$

are positive reals at all infinite places $\alpha^{-1}\left(v_{\infty}\right)$. Hence we may define

$$
h \in H o m_{\Omega_{q}}\left(R(G(L / K)), J^{*}(E)\right)
$$

by replacing $\alpha^{-1}\left(W_{K}(\alpha(\chi))\right)$ with $\alpha^{-1}(\sqrt{(N f(\alpha(\chi)))})$ in the definition of $W_{L / K}^{\prime}(\chi)_{\nu}$. The resulting class is equal to one at all finite places and is totally positive on symplectic representations at all infinite places. By Taylor [38], page 9 (see also Chinburg [3], page 19,(2.6.1) (proof)) $h$ is a determinant and represents the trivial element in $\mathcal{C L}(\mathrm{Z}[G(L / K)])$. Hence $W_{L / K}$ may be represented by $W_{L / K}^{\prime} \cdot h^{-1}$. However, when $\chi$ is irreducible and symplectic,

$$
\frac{\alpha^{-1}\left(W_{K}(\alpha(\chi))\right)}{\alpha^{-1}(\sqrt{(N f(\alpha(\chi)))})}=\frac{W_{K}(\chi)}{\sqrt{(N f(\chi))}}
$$


(Chinburg [5], page 327) so that the $\alpha^{-1}\left(v_{\infty}\right)$-component of this representative for $W_{L / K}$ is independent of $\alpha$ and hence does not depend on the choice of $v_{\infty}$.

\subsection{Local Chinburg Invariants}

We are now in a position to describe the local Chinburg invariants (c.f. Chinburg [4]). Let $L / K$ be a Galois extension of p-adic local fields. If $U \subset L^{*}$ is a $\mathbf{Z}[G(L / K)]$-submodule which is cohomologically trivial and $L^{*} / U$ is a finitely generated $\mathrm{Z}[G(L / K)]-$ module, then there is an exact sequence of the form

$$
L * / U \rightarrow A / U \rightarrow B \rightarrow \mathbf{Z}
$$

where $A$ and $B$ are cohomologically trivial, and both $A / U$ and $B$ are finitely generated $\mathbf{Z}[G(L / K)]$-modules. This is because $H^{2}\left(G(L / K) ; L^{*} / U\right) \cong E x t_{\mathbb{Z}[G(L / K)]}^{2}$ classifies such exact sequences as 2-extensions. This exact sequence is described in Snaith [32], Section 2.2.2, and represents the preimage of $[L: K]^{-1}$ under the natural canonical isomorphism inv : $H^{2}\left(G(L / K) ; L^{*} / U\right) \cong \mathrm{Z} /[L: K] \subset \mathrm{Q} / \mathrm{Z}$ (for more details on this cannonical embedding see Serre [28]). In any case, a finitely generated, cohomologically trivial $\mathrm{Z}[G(L / K)]$-module, $X$, has a finitely generated projective 
resolution of the form

$$
0 \rightarrow P_{1} \rightarrow P_{0} \longrightarrow X \rightarrow 0
$$

and therefore defines a class

$$
[X]=\left[P_{0}\right]-\left[P_{1}\right] \in \mathcal{C L}(\mathrm{Z}[G(L / K)])
$$

Therefore we have classes $[A / U]$ and $[B]$ in $\mathcal{C L}(\mathrm{Z}[G(L / K)])$, and we may define a class, which we shall call the local Chinburg invariant

$$
\Omega(L / K, U)=[A / U]-[B] \in \mathcal{C L}(\mathrm{Z}[G(L / K)])
$$

Proposition 1.4.1 (Snaith [92], page 47) The class $\Omega(L / K, U)$ is independent of the choice of $A$ and $B$ in the 2-extension

$$
L^{*} / U \rightarrow A / U \rightarrow B \rightarrow \mathbf{Z}
$$

Proof Let

$$
L^{*} / U \rightarrow A^{\prime} / U \rightarrow B^{\prime} \rightarrow \mathbf{Z}
$$

be another 2-extension, with $A^{\prime} / U, B^{\prime}$ finitely generated and $A^{\prime}, B^{\prime}$ cohomologically trivial, and which represents inv ${ }^{-1}\left([L: K]^{-1}\right) \in H^{2}\left(G(L / K) ; L^{*} / U\right)$. Therefore there is a commutative diagram resulting from the equivalence of these two 2extensions: 


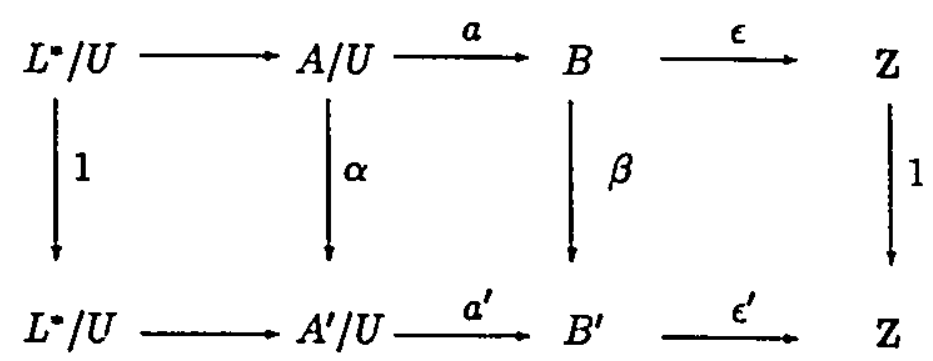

We may form a chain complex of $\mathrm{Z}[G(L / K)]$-homomorphisms

$$
0 \longrightarrow A / U \stackrel{(a, \alpha)}{\longrightarrow} B \oplus A^{\prime} / U \stackrel{\left(\beta_{1}-\alpha^{\prime}\right)}{\longrightarrow} B^{\prime} \longrightarrow 0
$$

It suffices to show that this sequence is exact, since exactness implies the relation

$$
[A / U]+\left[B^{\prime}\right]=\left[A / U \oplus B^{\prime}\right]=\left[B \oplus A^{\prime} / U\right]=[B]+\left[A^{\prime} / U\right] \in \mathcal{C L}(\mathbf{Z}[G(L / K)])
$$

However, at the left, if $(a, \alpha)(z)=(0,0)$ then $z \in L \cdot / U \subset A / U$ and then $0=\alpha(z)=$ z. Also, at the right, if $b^{\prime} \in B^{\prime}$ we may choose $b \in B$ such that $c(b)=\epsilon^{\prime}\left(b^{\prime}\right)$ and then $b^{\prime}-\beta(b) \in \operatorname{ker}\left(\epsilon^{\prime}\right)=i m\left(a^{\prime}\right)$. Finally, if $b \in B$ and $x \in A^{\prime} / U$ satisfy $\beta(b)=a^{\prime}(x)$ then $\epsilon(b)=\epsilon^{\prime}\left(a^{\prime}(x)\right)=0$ so that $b \in i m(a)$ and $b=a(y)$. Therefore

$$
\begin{aligned}
a^{\prime}(x-\alpha(y)) & =\beta(b)-a^{\prime}(\alpha(y)) \\
& =\beta(b)-\beta(a(y)) \\
& =0
\end{aligned}
$$


so that $x=\alpha(y)+w$ for some $w \in L^{*} / U$ and

$$
\begin{aligned}
(a, \alpha)(y+w) & =(u(y)+a(w), \alpha(y)+\alpha(w)) \\
& =(a(y), \alpha(y)+w) \\
& =(b, x)
\end{aligned}
$$

which establishes exactness in the middle.

\subsection{Global Chinburg Invariants}

Let $L / K$ be a finite Galois extension of number fields. In Chinburg [4] three classgroup invariants, $\Omega(L / K, i) \in \mathcal{C L}(\mathbf{Z}[G(L / K)])$, were constructed in a cohomological me'iner which is analogous to the construction of the local invariant of Section 1.4. We shall now give a definition of $\Omega(L / K, 2)$. This definition (Snaith [32]) is an adaptation of the original definition and is due to S. Kim [21] (see also Kim [22]) and is also used in Wilson [41].

For each finite prime $\mathcal{P} \triangleleft \mathcal{O}_{K}$, choose a prime $\mathcal{Q} \triangleleft \mathcal{O}_{L}$ lying over it, so that the decomposition group of $\mathcal{Q}$ is isomorphic to $G\left(L_{\mathcal{Q}} / K_{\mathcal{P}}\right)$. We shall say that $\mathcal{P}$ is tame if $L_{\mathcal{Q}} / K_{\mathcal{P}}$ is tamely ramified and that $\mathcal{P}$ is wild otherwise. By a theorem of $\mathrm{E}$. Noether (see [9]), if $\mathcal{P}$ is tame then $\mathcal{O}_{L_{\mathcal{Q}}}$ is a free $\mathcal{O}_{K_{\mathcal{P}}}\left[G\left(L_{\mathcal{Q}} / K_{\mathcal{P}}\right)\right]$-module of rank 
one. Therefore we may choose an adèle

$$
\left(a_{\mathcal{P}}\right)=a \in \prod_{\mathcal{P}} \mathcal{O}_{L_{\mathcal{Q}}}
$$

(the product being taken over finite primes of $K$ with $\mathcal{Q}$ being the chosen prime over $\mathcal{P})$ such that

(i) $a_{\mathcal{P}} \in \mathcal{O}_{L_{\mathcal{Q}}}$ and $K_{\mathcal{P}}\left[G\left(L_{\mathcal{Q}} / K_{\mathcal{P}}\right)\right] a_{\mathcal{P}}=L_{\mathcal{Q}}$ for each $\mathcal{P}$ and

(ii) $\mathcal{O}_{K_{\mathcal{P}}}\left[G\left(L_{\mathcal{Q}} / K_{\mathcal{P}}\right)\right] a_{\mathcal{P}}=\mathcal{O}_{L_{\mathcal{Q}}}$ for each tame $\mathcal{P}$.

There are isomorphisms, as in Section 1.3, of the form

$$
L \otimes_{K} K_{\mathcal{P}} \cong \prod_{R \mid \mathcal{P}} L_{\mathcal{R}} \cong \operatorname{Ind}_{G\left(L_{\mathcal{Q}} / K_{\mathcal{P}}\right)}^{G\left(L / K^{\mathcal{C}}\right)}\left(L_{\mathcal{Q}}\right)
$$

and

$$
\mathcal{O}_{L} \otimes_{\mathcal{O}_{K}} \mathcal{O}_{K_{\mathcal{P}}} \cong \prod_{\mathcal{R} \mid \mathcal{P}} \mathcal{O}_{L_{\mathcal{R}}} \cong \operatorname{Ind} G\left(L_{\mathcal{Q}}^{G(L / K)}\right)\left(\mathcal{O}_{L_{\mathbf{Q}}}\right)
$$

so that

(i) $\quad K_{\mathcal{P}}[G(L / K)] a_{\mathcal{P}} \cong \prod_{\mathcal{R} \mid \mathcal{P}} L_{\mathcal{R}}$ for each $\mathcal{P}$ and

(ii) $\mathcal{O}_{K_{\mathcal{P}}}[G(L / K)] a_{\mathcal{P}} \cong \prod_{\mathcal{R} \mid \mathcal{P}} \mathcal{O}_{L_{\mathcal{R}}}$ for each tame $\mathcal{P}$.

We will abbreviate $\mathcal{O}_{K_{\mathcal{P}}}\left[G\left(L_{\mathcal{Q}} / K_{\mathcal{P}}\right)\right] a_{\mathcal{P}}$ to $X_{\mathcal{Q}}$ and set $X=\mathcal{O}_{K}[G(L / K)] a$. This is to be interpreted as meaning that $X$ is the intersection of $L$ with the product of its $\mathcal{P}$-completions, $X_{\mathcal{P}}$, where both are considered as subgroups of the adèles. Hence 
$X$ is a locally free $\mathcal{O}_{K}[G(L / K)]$-module whose $\mathcal{P}$-completion is

$$
X_{\mathcal{p}}=\mathcal{O}_{K_{\mathcal{P}}}[G(L / K)] a_{\mathcal{P}}=\operatorname{Ind} d_{G\left(L_{\mathcal{Q}} / K_{\mathcal{P}}\right)}^{G(L / K)}\left(X_{\mathcal{Q}}\right)
$$

In addition, we shall assume henceforth (by replacing $X$ by $m X$ for a suitable integer, $m \in Z$, if necessary) that the $\mathcal{Q}$-adic exponential defines an isomorphism

$$
\exp : X_{\mathcal{Q}} \stackrel{\cong}{\rightarrow} 1+X_{\mathcal{Q}} \subset \mathcal{O}_{L_{\mathcal{Q}}}^{*}
$$

for all wild $L_{\mathcal{Q}} / K_{\mathcal{P}}$.

Since $X$ is locally free, it is cohomologically trivial and so also is $X_{\mathcal{Q}}$ for each $\mathcal{Q}$. Hence $X$ defines a class in $\mathcal{C L}(Z[G(L / K)])$. Therefore we may define an element, which we shall call the second (global) Chinburg invariant, by the formula

$$
\Omega(L / K, 2)=[X]+\sum_{\mathcal{P} \text { wild }} \operatorname{Ind} d_{G\left(L_{\mathbf{Q}} / K_{\mathcal{P}}\right)}^{G(L / K)}\left(\Omega\left(L_{\mathcal{Q}} / K_{\mathcal{P}}, 1+X_{\mathcal{Q}}\right)\right)
$$

in $\mathcal{C L}(\mathrm{Z}[G(L / K)])$. Here $\Omega\left(L_{\mathcal{Q}} / K_{\mathcal{P}}, 1+X_{\mathcal{Q}}\right)$ is the local Chinburg invariant of Section 1.4. The assumptions of Section 1.5 ensure that $1+X_{\mathcal{Q}}$ is cohomologically trivial.

Proposition 1.5.1 The definition of $\Omega(L / K, 2)$ is independent of the choice of $X$, satisfying the conditions of Section 1.5, (i) and (ii).

Proof Suppose first that $Y$ is another choice of lattice and that $X \subset Y$. In the 
notation of Section 1.5, one easily obtains the following relations in the class group:

$$
\begin{aligned}
& {[Y]-[X]=[Y / X]} \\
& =\Sigma_{\mathcal{P}} \text { wild }\left[Y_{\mathcal{P}} / X_{\mathcal{P}}\right] \\
& =\Sigma_{\mathcal{P}} \text { wild }\left[Y_{\mathcal{P}}\right]-\left[X_{\mathcal{P}}\right] \\
& =\Sigma_{\mathcal{P}} \text { wild } \operatorname{Ind}_{\mathrm{Z}\left[G\left(L_{\boldsymbol{Q}} / K_{\mathcal{P}}\right)\right]}^{\mathrm{Z}[G / K)]}\left(\left[Y_{\boldsymbol{Q}}\right]-\left[X_{\mathbf{Q}}\right]\right) \\
& =\Sigma_{\mathcal{P}} \text { wild } \operatorname{Ind}_{\mathbf{z}\left[G\left(L_{\mathcal{Q}} / K_{\mathcal{P}}\right)\right]}^{Z\left[G\left(L / K^{\prime}\right)\right]}\left(\Omega\left(L_{\mathcal{Q}} / K_{\mathcal{P}}, 1+Y_{\mathcal{Q}}\right)\right. \\
& \left.-\Omega\left(L_{Q} / K_{P}, 1+X_{Q}\right)\right) .
\end{aligned}
$$

This proves the result when $X \subset Y$. Given two general lattices $X$ and $Y$, we may find a third lattice $W$, which lies in $X \cap Y$ and may apply the preceding argument to the pairs, $(X, W)$ and $(Y, W)$ to complete the proof.

Remark 1.5.2 When $L_{\mathbb{Q}} / K_{p}$ is tame for all $\mathcal{P}$ we say that $L / K$ is tame. Observe that

$$
\Omega(L / K, 2)=\left[\mathcal{O}_{L}\right] \in \mathcal{C L}(\mathbf{Z}[G(L / K)])
$$

when $L / K$ is tame.

We are now in a position to state the Second Chinburg Conjecture (see Chinburg $[3,4,5])$. 
Conjecture 1.5.3 (The Second Chinlurg Conjecture) Let $L / K$ be a finite Galois extension of number fields. Then

$$
\Omega(L / K, 2)=W_{L / K} \in \mathcal{C} \mathcal{L}(\mathrm{Z}[G(L / K)])
$$

This conjecture has a considerable amount of supporting evidence. In the first place it generalises, by Remark 1.5.2, a conjecture of Fröhlich which states, if $L / K$ is tamely ramified, that

$$
\left[\mathcal{O}_{L}\right]=W_{L / K} \in \mathcal{C L}(\mathrm{Z}[G(L / K)])
$$

This case was proved by M.J. Taylor [39] and is also described in Fröhlich [9]. A sketch of a slightly different proof of Taylor's result can be found in Snaith [32], Chapter 3.

As to further evidence, several wildly ramified examples have been verified for $K=\mathrm{Q}$. The case where $\operatorname{Gal}(L / \mathrm{Q}) \cong Q_{8}$ and the prime 2 is not totally ramified, is treated in Kim [21, 22] (see also Snaith [32], Chapter 6, for a new proof). The case where $L$ is a p-cyclotomic extension of degree $p^{n}$ over $\mathbf{Q}$, for an odd regular prime $p$, is proved in Snaith [31]. Recently, C. Greither [17] has shown that this conjecture holds for all abelian extensions of $\mathbf{Q}$ with odd conductor. Apart from this direction, M. Rogers has proved that $\Omega(L / K, 2)-W_{L / K}$ is in the kernel of the map from $\mathcal{C L}(\mathrm{Z}[G(L / K)])$ to $G_{0}(\mathrm{Z}[G(L / K)])$, the Grothendieck group of all finitely 
generated $\mathrm{Z}[G(L / K)]$-modules. In fact, (Chinburg [5], page 327) $D(\mathrm{Z}[G(L / K)])$ is contained in this kernel and D. Holland [19] (see Snaith [33], Chapter 7, for a new proof) has shown the stronger resuli that

$$
\Omega(L / K, 2)-W_{L / K} \in D(\mathbf{Z}[G(L / K)])
$$

In the remaining chapters of this thesis, we adopt the techniques of Snaith [32] to show that the Second Chinburg Conjecture also holds for two familics of quaternion extensions of $Q$ in which the prime 2 is totally ramified. 


\section{Chapter 2}

\section{Local Quaternion Extensions}

\subsection{The Two Extensions}

Let $N / \mathrm{Q}$ be a quaternion extension, i.e. a normal extension with Galois group isomorphic to the quaternion group of order eight

$$
Q_{8}=\left\{x, y \mid x^{2}=y^{2}, y^{4}=1, x y x^{-1}=y^{-1}\right\},
$$

such that the prime 2 is totally ramified i.e. there is only one prime of $\mathcal{O}_{L}$ over the prime 2 of $Q$ whose ramification index is 8 .

In this section we are going to study two local quaternion extensions $L / Q_{2}$ which are totally ramified. There are exactly four such extensions (see Jensen and Yui [20], 
Kim [22], and Snaith [32]).

Lemma 2.1.1 Let $N$ be as above and let $H$ be the bi-quadratic subfield of $N$. Suppose that $N_{v}$ contains $\mathrm{Q}_{2}(\sqrt{3}, \sqrt{10})$ where $v$ is a prime of $N$ above the prime 2. Then $H=\mathrm{Q}\left(\sqrt{d_{1}}, \sqrt{d_{2}}\right)$ where $d_{1}, d_{2}$ are square-free integers, $d_{1} \equiv 3(\bmod 8)$, and $d_{2} \equiv 10 \quad(\bmod 16)$

Proof Without loss of generality we may assume that $d_{1}$ is odd. Since the prime 2 is totally ramified, $d_{1} \equiv 3(\bmod 8)$. It follows from Fröhlich [14], Theorem 5 , that $d_{2} \equiv 2(\bmod 8)$. This means that either $d_{2} \equiv 2(\bmod 16)$ or $d_{2} \equiv 10$ $(\bmod 16)$. From the given hypothesis, we have that $N_{v}$ contains $\mathbf{Q}_{2}(\sqrt{10})$, so $d_{2} \equiv 10 \quad(\bmod 16)$

Throughout this chapter, we let $E=\mathrm{Q}_{2}(\sqrt{3}, \sqrt{10})$. As usual, we use $\pi_{F}$ to denote a prime element of the local field $F$. We let

$$
L_{ \pm}=E\left(\alpha_{ \pm}\right)
$$

where

$$
\alpha_{ \pm}^{2}= \pm(1+\sqrt{3}+\sqrt{10} / 10+\sqrt{30} / 10)
$$

We are now in a position to prove the following lemma. 
Lemma 2.1.2 If $L=L_{ \pm}$and $\alpha=\alpha_{ \pm}$then $L$ is a quaternion extension of $\mathbf{Q}_{2}$ containing $E, \pi=\pi_{L}=1+\alpha$, and the Galois action of $Q_{8}$ on $L / Q_{2}$ is given by the following formulae.

$$
\begin{array}{ll}
x(\alpha)=(1-\sqrt{10}) \alpha / 3, & x(\sqrt{10})=-\sqrt{10} \\
y(\alpha)=(\sqrt{3}-1) \alpha / \sqrt{30} v, & y(\sqrt{10})=\sqrt{10} \\
x(\sqrt{3})=\sqrt{3}, & y(\sqrt{3})=-\sqrt{3}
\end{array}
$$

where $v \in Q_{2}^{*}$ and $v^{2}=-1 / 15$.

Proof It follows from Jensen and Yui [20] that $L_{ \pm} / Q_{2}$ are precisely the two non-isomorphic quaternion extensions of $\mathbf{Q}_{\mathbf{2}}$ which contain the biquadratic subfield $E=\mathrm{Q}_{2}(\sqrt{3}, \sqrt{10})$. It remains to show that the defining relations for $\mathbf{Q}_{8}$ holds for the action on $\alpha$. We consider the following elementary computations:

$$
\begin{aligned}
x^{2}(\alpha) & =(1+\sqrt{10}) x(\alpha) / 3 \\
& =(1+\sqrt{10})(1-\sqrt{10}) \alpha / 9 \\
& =-\alpha \\
y^{2}(\alpha) & =(-\sqrt{3}-1) y(\alpha) /(-\sqrt{30} v) \\
& =(\sqrt{3}+1)(\sqrt{3}-1) \alpha /\left(30 v^{2}\right) \\
& =-\alpha
\end{aligned}
$$


and

$$
\begin{aligned}
x y x(\alpha) & =x y((1-\sqrt{10}) \alpha / 3) \\
& =x((1-\sqrt{10}) / 3(\sqrt{3}-1) \alpha / \sqrt{30} v) \\
& =-(1+\sqrt{30})(\sqrt{3}-1)(1-\sqrt{10}) \alpha / 3 \sqrt{30} v \\
& =(\sqrt{3}-1) \alpha / \sqrt{30} v \\
& =y(\alpha) .
\end{aligned}
$$

To show $\pi=\pi_{L}=1+\alpha$ we abserve that both $N_{L / Q_{2}}\left(1+\alpha_{+}\right)=166 / 25$ and $N_{L / Q_{2}}\left(1+\alpha_{-}\right)=6 / 25$ have valuation 1 in $\mathrm{Q}_{2}$.

\subsection{The Fundamental 2-extension}

Let $K_{n r}$ denote the maximal unramified extension of $K$. Since $L_{ \pm}$is totally ramified we have $L_{ \pm} \cap Q_{2, n r}=Q_{2}$. As in Snaith [32], Section 1.2 (see Serre [28], page 202; and also Snaith [33]), letting $\mathcal{F}$ denote the Frobenius automorphism of $\mathbf{Q}_{2, \mathrm{nr}} / \mathbf{Q}_{\mathbf{2}}$ and $L_{0}=L_{ \pm} Q_{2, n r}$, we have the following 2-extension:

$$
L_{ \pm}^{*} \longrightarrow L_{0}^{*} \stackrel{F / 1}{\longrightarrow} L_{0}^{*} \longrightarrow \mathbf{Z}
$$

where $F$ denotes the automorphism of $L_{0}$ which is the identity on $L$ and is equal to $\mathcal{F}$ on $Q_{2, n r}$. The existence of the above sequence implies the existence of

$$
t_{1}, t_{2} \in L_{0}^{*}
$$


such that

$$
\left\{\begin{array}{l}
F\left(\pi t_{1}\right) / \pi t_{1}=y(\pi) / \pi \in L^{*} \\
F\left(t_{2}\right) / t_{2}=x(\pi) / \pi \in L^{*}
\end{array}\right.
$$

We observe that the above relations are not changed when we multiply $t_{1}$ or $t_{2}$ by any element of $L^{*}$.

As in Snaith [32], Section 6.1.4, we introduce the following commutative diagram:

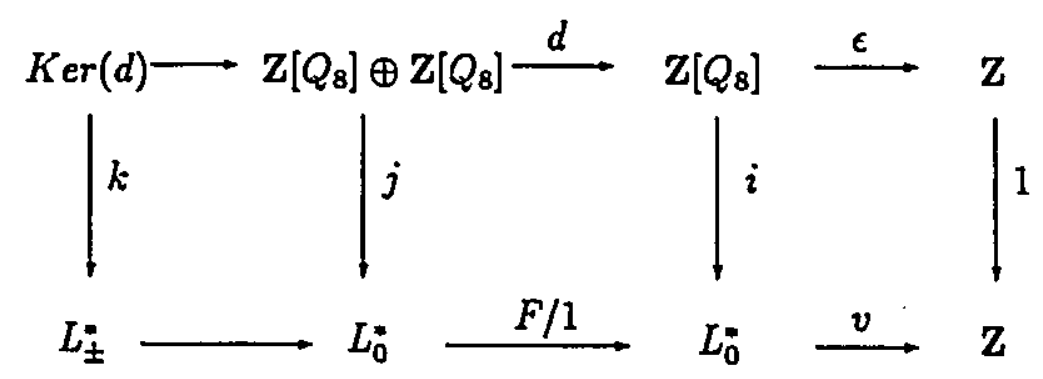

Here the upper line is the beginning of the standard, periodic $\mathbf{Z}\left[Q_{8}\right]$-resolution of $\mathbf{Z}$. Hence, if $b, b^{\prime}$ are the two free generators ( see Snaith [34], page 19), then $d(b)=x-1$ and $d\left(b^{\prime}\right)=y-1$. Hence $\operatorname{Ker}(d)$ is the $\mathbf{Z}\left[Q_{8}\right]$-submodule generated by

$$
c_{1}=(1+x) b-(1+y) b^{\prime}
$$

and

$$
c_{2}=(x y+1) b+(x-1) b^{\prime}
$$

In the upper line, the map $\epsilon$ is defined by $\epsilon(g)=1$ for $g \in Q_{8}$. The map $i$ is given by $i(1)=\pi \in L^{*} \subset L_{0^{*}}^{*}$ The map $j$ is defined by $j(b)=t_{2}$ and $j\left(b^{\prime}\right)=\pi t_{1}$. The 
homomorphism $k$ is determined by the following formulac:

$$
k\left(c_{1}\right)=t_{2} x\left(t_{2}\right)\left(\pi t_{1} y\left(\pi t_{1}\right)\right)^{-1}
$$

and

$$
k\left(c_{2}\right)=t_{2} x y\left(t_{2}\right) x\left(\pi t_{1}\right)\left(\pi t_{1}\right)^{-1}
$$

The map $k$ plays a very important role in our study. In the next section we shall show that $k$ is injective. The strategy of our proof of the injectivity of $k$ will be explained later in the proof of Theorem 2.3.8.

We now find some elements of $L_{0}$ which depend on $t_{1}$ and $t_{2}$ but belong to $L^{*}$. The following Lemma will be used very often in Section 2.3.

Lemma 2.2.1 For $t_{1}$ and $t_{2}$ defined above, we have the following.

$$
\begin{aligned}
& \text { (1) }\left(1+x+x^{2}+x^{3}\right)\left(t_{2}\right) \in \mathrm{Q}_{2}(\sqrt{3}), \\
& \text { (2) }\left(1+x+x^{2}+x^{3}\right)(1+y)\left(t_{2}\right) \in \mathrm{Q}_{2}, \\
& \text { (3) }\left(1+y+y^{2}+y^{3}\right)\left(t_{1}\right) \in \mathrm{Q}_{2}(\sqrt{10}), \\
& \text { (4) }\left(1+x y+x^{2}+(x y)^{3}\right)\left(t_{2} / \pi t_{1}\right) \in \mathrm{Q}_{2}(\sqrt{30}),
\end{aligned}
$$

and

$$
\text { (5) } k\left(\left(1-x^{2}\right)\left(c_{1}-c_{2}\right)\right) \in L^{*} \text {. }
$$

Proof Let $\sigma_{1}=1+x+x^{2}+x^{3}$. Since $\sigma_{1}=N_{L_{0} / Q_{2, \operatorname{Mr}}(\sqrt{3})}$, we have $\sigma_{1}\left(t_{2}\right) \epsilon$ $\mathbf{Q}_{2, n r}(\sqrt{3})$, and since $\mathbf{Q}_{2, n r}(\sqrt{3}) \cap L=\mathbf{Q}_{2}(\sqrt{3})$, to show (1) it suffices to show that 
$\sigma_{1}\left(t_{2}\right) \in L$, i.e. we have to show that $(F / 1)\left(\sigma_{1}\left(t_{2}\right)\right)=1$. We consider

$$
\begin{aligned}
(F-1)\left(\sigma_{1}\left(t_{2}\right)\right) & =\sigma_{1}\left(F\left(t_{2}\right)\right) / \sigma_{1}\left(t_{2}\right) \\
& =\left(F\left(t_{2}\right) / t_{2}\right) x\left(F\left(t_{2}\right) / t_{2}\right) x^{2}\left(F\left(t_{2}\right) / t_{2}\right) x^{3}\left(F\left(t_{2}\right) / t_{2}\right) \\
& =(x(\pi) / \pi)\left(x^{2}(\pi) / x(\pi)\right)\left(x^{3}(\pi) / x^{2}(\pi)\right)\left(x^{4}(\pi) / x^{3}(\pi)\right) \\
& =1 .
\end{aligned}
$$

Thus (1) is now proved. Statements (2), (3), (4), and (5) are proved in the same manner.

The following arguments will give the images of $\pi$ under the actions of $x, y$ and $x y$.

Since $L / Q_{2}$ is totally ramified, we have $v(a)=v_{L}(a)=v_{Q_{2}}\left(N_{L / Q_{2}}(a)\right)$ for all $a \in L^{*}$. Hence, to express a unit $a$ of $L$ in terms of $\pi$ we first have to compute $v(a-1)$. If $v(a-1)=m$, then $a=1+\pi^{m}+\cdots$. The value of $v\left(a-1-\pi^{m}\right)$ will determine the next term for $a$, etc. With this observation, using Maple we have the following identities. For $\alpha=\alpha_{+}$,

$$
\begin{aligned}
x(\pi)= & \pi\left(1+\pi^{3}+\pi^{4}+\pi^{5}+\pi^{6}+\pi^{9}+\pi^{10}+\pi^{16}+\cdots\right), \\
y(\pi)= & \pi\left(1+\pi+\pi^{2}+\pi^{3}+\pi^{4}+\pi^{7}+\pi^{9}+\pi^{10}+\pi^{13}+\right. \\
& \left.\pi^{14}+\pi^{15}+\pi^{17}+\cdots\right), \\
x y(\pi)= & \pi\left(1+\pi+\pi^{2}+\pi^{8}+\pi^{12}+\pi^{13}+\pi^{14}+\pi^{15}+\cdots\right)
\end{aligned}
$$


and for $\alpha=\alpha_{-}$,

$$
\begin{aligned}
x(\pi)= & \pi\left(1+\pi^{3}+\pi^{4}+\pi^{5}+\pi^{6}+\pi^{9}+\pi^{10}+\pi^{11}+\pi^{12}+\right. \\
& \left.\pi^{13}+\pi^{14}+\pi^{15}+\pi^{17}+\cdots\right), \\
y(\pi)= & \pi\left(1+\pi+\pi^{2}+\pi^{3}+\pi^{4}+\pi^{8}+\pi^{11}+\pi^{12}+\pi^{15}+\right. \\
& \left.\pi^{16}+\cdots\right), \\
x y(\pi)= & \pi\left(1+\pi+\pi^{2}+\pi^{7}+\pi^{9}+\pi^{10}+\pi^{11}+\pi^{15}+\pi^{16}+\cdots\right) .
\end{aligned}
$$

\subsection{The Injectivity of $k$}

We start this section with the notations of the following four important elements of $\mathbf{Z}\left[Q_{8}\right]:$

$$
\begin{aligned}
& \sigma=\left(1+x+x^{2}+x^{3}\right)(1+y), \\
& \tau=\left(1+x+x^{2}+x^{3}\right)(1-y), \\
& \lambda=\left(1+y+y^{2}+y^{3}\right)(1-x)
\end{aligned}
$$

and

$$
\rho=\left(1+x y+x^{2}+(x y)^{3}\right)(1-x) .
$$

Unless stated otherwise, throughout this chapter we let $K=\mathrm{Q}_{2}(\sqrt{3}), V=$ $\mathbf{Q}_{2}(\sqrt{10})$ and $W=\mathbf{Q}_{2}(\sqrt{30})$. It is easy to see that we may choose $\pi_{K}=1+\sqrt{3}$, 
$\pi_{V}=\sqrt{10}$ and $\pi_{W}=\sqrt{30}$. With the above notation, $\operatorname{Gal}(L / K), \operatorname{Gal}(L / V)$ and $\operatorname{Gal}(L / W)$ can be identified with $\langle x\rangle,\langle y\rangle$, and $\langle x y\rangle$ respectively.

The purpose of this section is to prove that the map $k$, defined as in the previous section, is injective using some suitable choices for $t_{1}, t_{2}$, and their images under the actions of $\sigma, \tau, \lambda$, and $\rho$. From the previous section we know that $x(\pi) / \pi \in U_{L}^{3}$ and $y(\pi) / \pi \in U_{L}^{1}$. It follows from Serre [28], Proposition 15, Chapter 13, that $t_{2} \in U_{L_{0}}^{3}$ and $t_{1} \in U_{L_{0}}^{1}$. This allows us to write

$$
t_{2}=\left(1+a_{3} \pi^{3}\right)\left(1+a_{4} \pi^{4}\right)\left(1+a_{5} \pi^{5}\right) u_{6}
$$

where $u_{6} \in U_{L_{0}}^{6}, a_{3}, a_{4}$ and $a_{5}$ are either 0 or roots of unity of odd order in $\mathbf{Q}_{2, n \text { r }}$.

Using the facts that $F\left(t_{2}\right) / t_{2}=x(\pi) / \pi$ and $F\left(1+a_{i} \pi^{i}\right)=\left(1+a_{i}^{2} \pi^{i}\right)$ for $i=3,4,5$, we have

$$
a_{i}^{2}+a_{i}+1 \equiv 0 \quad(\bmod 2)
$$

Hence $a_{i}$ must be a primitive cube root of unity, i.e. $a_{i}=\xi$ or $\xi^{2}$ where $\xi \neq 1$ and $\xi^{3}=1$. We may multiply $t_{2}$ by $1+\pi^{i}$ if necessary to assume that $a_{i}=\xi$ for all $i=3,4,5$. Thus $t_{2}$ may be chosen to be of the form

$$
t_{2}=\left(1+\xi \pi^{3}\right)\left(1+\xi \pi^{4}\right)\left(1+\xi \pi^{5}\right) u_{6}
$$

Using similar arguments, we may assume that $t_{1}=(1+\xi \pi) u_{2}$ with $u_{2} \in U_{L_{0}}^{2}$. Before going further in this direction, we prove the following useful result. 
Proposition 2.3.1 For $\sigma$ defined as in the beginning of this section and $u=0$ or an odd root of unity, we have the following.

(1) $\left(1+x+x^{2}+x^{3}\right)\left(1+u \pi^{n}\right) \equiv 1 \quad\left(\bmod \mathcal{P}_{K_{0}}^{8}\right)$ for all $n \geq 16$

(2) $\sigma\left(1+u \pi^{n}\right) \equiv 1 \quad(\bmod 32)$ for all $n \geq 16$

(3) $\left(1+y+y^{2}+y^{3}\right)\left(1+u \pi^{n}\right) \equiv 1 \quad\left(\bmod \mathcal{P}_{V_{0}}^{6}\right)$ for all $n \geq 12$

and

(4) $\left(1+x y+x^{2}+(x y)^{3}\right)\left(1+u \pi^{n}\right) \equiv 1 \quad\left(\bmod \mathcal{P}_{W_{0}}^{6}\right)$ for all $n \geq 12$

Proof To prove statement (1), we observe that $1+x+x^{2}+x^{3}=(1+x)\left(1+x^{2}\right)$. Recall from Section 2.1 that $E$ is defined to be the bi-quadratic subfield $Q_{2}(\sqrt{3}, \sqrt{10})$ of $L$. We consider

$$
\left(1+x^{2}\right)\left(1+u \pi^{n}\right)=1+u T r_{L_{0} / E_{0}}\left(\pi^{n}\right)+u^{2} N_{L_{0} / E_{0}}\left(\pi^{n}\right)
$$

Since $v_{L_{0}}\left(x^{2}(\pi) / \pi-1\right)=7$, it follows from Serre [28], page 83 , that for $n \geq 16$, $\operatorname{Tr}_{L_{0} / E_{0}}\left(\pi^{n}\right) \in \mathcal{P}_{E_{0}}^{12}$. Together with the fact that $N_{L_{0} / E_{0}}\left(\pi^{n}\right) \in \mathcal{P}_{E_{0}}^{n}$, we have

$$
\left(1+x^{2}\right)\left(1+u \pi^{n}\right) \equiv 1 \quad\left(\bmod \mathcal{P}_{E_{0}}^{8}\right) \text { for all } n \geq 8
$$

Since $v_{E_{0}}\left(x\left(\pi_{E_{0}}\right) / \pi_{E_{0}}-1\right)=3$, using similar arguments for the extension $E_{0} / K_{0}$, we conclude that

$$
\left(1+x+x^{2}+x^{3}\right)\left(1+u \pi^{n}\right) \equiv 1 \quad\left(\bmod \mathcal{P}_{K_{0}}^{8}\right)
$$


Statement (2) is proved by applying the above results and using the facts that $v_{K_{0}}\left(y\left(\pi_{K_{0}}\right) / \pi_{K_{0}}\right)=1$.

To prove statements (3) and (4) we use identical arguments and the following facts:

$$
\begin{aligned}
1+y+y^{2}+y^{3} & =(1+y)\left(1+x^{2}\right) \\
1+x y+x^{2}+(x y)^{3} & =(1+x y)\left(1+x^{2}\right)
\end{aligned}
$$

$v_{E_{0}}\left(y\left(\pi_{E_{0}}\right) / \pi_{E_{0}}-1\right)=1$ and $v_{E_{0}}\left(x y\left(\pi_{E_{0}}\right) / \pi_{E_{0}}-1\right)=1$.

To obtain more information about $t_{2}$ we let

$$
t_{2}=\left(1+\xi \pi^{3}\right)\left(1+\xi \pi^{4}\right)\left(1+\xi \pi^{5}\right)\left(\prod_{i=6}^{15}\left(1+a_{i} \pi^{i}\right)\right) u_{16}
$$

where $u_{16} \in U_{L_{0}}^{16}$ and $a_{i}$ is either 0 or an odd root of unity in $Q_{2, n r}$, and prove the following lemma.

Lemma 2.3.2 The element, $t_{2}$, may be chosen so that

$$
\sigma\left(t_{2}\right)=\left(1+x+x^{2}+x^{3}\right)(1+y)\left(t_{2}\right) \equiv 13(\bmod 32) .
$$

Proof Using the facts that

$$
F\left(1+z \pi^{m}\right) /\left(1+z \pi^{m}\right)=\left(1+z^{2} \pi^{m}\right)\left(1-z \pi^{m}+z^{2} \pi^{2 m}-z^{3} \pi^{3 m}+\cdots\right)
$$


where $z$ is either 0 or an odd root of unity lying in $Q_{2, n r}$, and for $\alpha=\alpha_{+}$,

$$
F\left(t_{2}\right) / t_{2}=x(\pi) / \pi=1+\pi^{3}+\pi^{4}+\pi^{5}+\pi^{6}+\pi^{9}+\pi^{10}+\pi^{16}+\cdots
$$

we have the following identities, modulo 2,

$$
\begin{aligned}
a_{6}+a_{6}^{2}+\xi^{2} & \equiv 0 \\
a_{7}+a_{7}^{2}+1 & \equiv 0 \\
a_{8}+a_{8}^{2}+\xi^{2} & \equiv 0 \\
a_{9}+a_{9}^{2} & \equiv 0 \\
a_{10}+a_{10}^{2}+\xi^{2} & \equiv 0 \\
a_{11}+a_{11}^{2}+\xi & \equiv 0 \\
a_{12}+a_{12}^{2}+a_{6}+a_{6}^{3} & \equiv 0 \\
a_{13}+a_{13}^{2}+\xi & \equiv 0 \\
a_{14}+a_{14}^{2}+\gamma+a_{8}^{3}+1 & \equiv 0
\end{aligned}
$$

and

$$
a_{15}+a_{15}^{2}+1 \equiv 0
$$

where $2 \gamma \equiv a_{6}+a_{6}^{2}+\xi^{2} \quad(\bmod 4)$. In this case we may choose $a_{7}=\xi$ and $a_{\theta}=0$. 
We observe that, modulo 2,

$$
\left(a_{6}+a_{8}\right)+\left(a_{6}+a_{8}\right)^{2} \equiv\left(a_{6}+a_{6}^{2}\right)+\left(a_{8}+a_{8}^{2}\right) \equiv \xi^{2}+\xi^{2} \equiv 0 .
$$

Thus modulo $2, a_{6}+a_{8} \equiv 0$ or 1 , and multiplying $t_{2}$ by $1+\pi^{8}$ if necessary, we may assume $a_{6}+a_{8} \equiv 0 \quad(\bmod 2)$. For the same reason, we may choose $a_{10}$ and $a_{11}$ so that $a_{10}+a_{11}+\xi^{2} \equiv 0 \quad(\bmod 2)$.

To compute $\sigma\left(t_{2}\right)$ modulo 32 we observe that

$$
\begin{aligned}
& \sigma\left(1+\xi \pi^{3}\right) \equiv 1+4+16 \xi, \\
& \sigma\left(1+\xi \pi^{4}\right) \equiv 1+16+16 \xi, \\
& \sigma\left(1+\xi \pi^{5}\right) \equiv 1+8+8 \xi, \\
& \sigma\left(1+a_{6} \pi^{6}\right) \equiv 1+24 a_{6}+8 a_{6}^{2}, \\
& \sigma\left(1+\xi \pi^{7}\right) \equiv 1+8+16 \xi, \\
& \sigma\left(1+a_{8} \pi^{8}\right) \equiv 1+16 a_{81}^{2}, \\
& \sigma\left(1+a_{9} \pi^{9}\right) \equiv 1, \\
& \sigma\left(1+a_{10} \pi^{10}\right) \equiv 1+16 a_{10}+16 a_{10}^{2}, \\
& \sigma\left(1+a_{11} \pi^{11}\right) \equiv 1+16 a_{11}+16 a_{11}^{2}, \\
& \sigma\left(1+a_{12} \pi^{12}\right) \equiv 1+16 a_{12},
\end{aligned}
$$




$$
\begin{aligned}
& \sigma\left(1+a_{13} \pi^{15}\right) \equiv 1+16 a_{13}, \\
& \sigma\left(1+a_{14} \pi^{14}\right) \equiv 1+16 a_{14}, \\
& \sigma\left(1+a_{15} \pi^{15}\right) \equiv 1+16 a_{15}
\end{aligned}
$$

and $\sigma\left(1+u \pi^{n}\right) \equiv 1 \quad(\bmod 32)$ for all $n \geq 16$, and $u=0$ or an odd root of unity (by Proposition 2.3.1(2)). Using all the above identities together with results of 2.2.1(2), we have, modulo 32 ,

$$
\sigma\left(t_{2}\right) \equiv 13+16\left(\gamma+\xi+a_{12}+a_{13}+a_{14}+a_{15}\right)
$$

is an element of $\mathbf{Z}_{2}$. In the case $\alpha=\alpha_{-}$, we use similar arguments and obtain the same formula for $\sigma\left(t_{2}\right)$. Since $\sigma\left(t_{2}\right) \in \mathbf{Z}_{2}^{*}$, modulo 2 ,

$$
\gamma+\xi+a_{12}+a_{13}+a_{14}+a_{15} \equiv 0 \text { or } 1 \text {. }
$$

Multiplying $t_{2}$ by $1+\pi^{14}$ if necessary, we may assume that the above identity is 0 . Therefore,

$$
\sigma\left(t_{2}\right)=(1+y)\left(1+x+x^{2}+x^{3}\right)\left(t_{2}\right) \equiv 13(\bmod 32),
$$

as desired.

Corollary 2.3.3 With the choice of $t_{2}$ as in Lemma 2.3.2,

$$
\left(1+x+x^{2}+x^{3}\right)\left(t_{2}\right) \equiv 1+\pi_{K}^{3}+\pi_{K}^{4}+\pi_{K}^{5}+\pi_{K}^{7}\left(\bmod \mathcal{P}_{K}^{8}\right)
$$


where $\pi_{K}=1+\sqrt{3}$ is a prime element of the field $K=\mathrm{Q}_{2}(\sqrt{3})$.

Proof As in the proof of Lemma 2.3.2, if we let $\sigma_{1}=1+x+x^{2}+x^{3}$, then direct computations show that, modulo $\mathcal{P}_{K_{0}}^{8}$,

$$
\begin{gathered}
\sigma_{1}\left(1+\xi \pi^{3}\right) \equiv 15+8 \xi+6 \sqrt{3}+12 \xi \sqrt{3} \\
\sigma_{1}\left(1+\xi \pi^{4}\right) \equiv 1+8 \xi+4 \sqrt{3} \\
\sigma_{1}\left(1+\xi \pi^{5}\right) \equiv 5+12 \xi \\
\sigma_{1}\left(1+a_{6} \pi^{6}\right) \equiv 1+12 a_{6}+4 a_{6}^{2}+\left(12 a_{6}+12 a_{6}^{2}+8 a_{6}^{4}\right) \sqrt{3} \\
\sigma_{1}\left(1+\xi \pi^{7}\right) \equiv 1+8 \xi+12 \sqrt{3} \\
\sigma_{1}\left(1+a_{8} \pi^{8}\right) \equiv 1+a_{8}^{2}+a_{8} \sqrt{3} \\
\sigma_{1}\left(1+a_{9} \pi^{8}\right) \equiv 1 \\
\sigma_{1}\left(1+a_{10} \pi^{10}\right) \equiv 1+8 a_{10}+8 a_{10}^{2}+8 a_{10}^{2} \sqrt{3} \\
\sigma_{1}\left(1+a_{11} \pi^{11}\right) \equiv 1+8 a_{11}+8 a_{11}^{2}+8 a_{11}^{2} \sqrt{3} \\
\sigma_{1}\left(1+a_{12} \pi^{12}\right) \equiv 1+8 a_{12}+8 a_{12} \sqrt{3} \\
\sigma_{1}\left(1+a_{13} \pi^{13}\right) \equiv 1+8 a_{13}+8 a_{13} \sqrt{3} \\
\sigma_{1}\left(1+a_{14} \pi^{14}\right) \equiv 1+8 a_{14}+8 a_{14} \sqrt{3} \\
\sigma_{1}\left(1+a_{15} \pi^{15}\right) \equiv 1+8 a_{18}+8 a_{15} \sqrt{3}
\end{gathered}
$$

and $\sigma_{1}\left(1+u \pi_{K}^{n}\right) \equiv 1\left(\bmod \mathcal{P}_{K_{0}}^{8}\right)$ for all $n \geq 16$, and $u=0$ or an odd root of unity (by Proposition 2.3.1(1)). Multiplying the above and using the result of Lemma 
2.2.1(1) and the identities of Lemma 2.3.2, we have, modulo $\mathcal{P}_{K^{*}}^{8}$,

$$
\left(1+x+x^{2}+x^{3}\right)\left(t_{2}\right) \equiv 1+\pi_{K^{-}}^{3}+\pi_{K^{4}}^{4}+\pi_{K^{+}}^{5}+C \pi^{6}+D \pi^{7}
$$

where for $\gamma$ defined as in Lemma 2.3.3, $C \equiv \xi^{2}+a_{10}+a_{11}(\bmod 2)$ and $D \equiv$ $\gamma+a_{6}+a_{8}^{2}+a_{12}+a_{13}+a_{14}+a_{15}(\bmod 2)$. Recall that in the proof of the last lemma we chose the coefficients so that, modulo $2, a_{6}+a_{8}^{2} \equiv a_{6}+a_{6}^{2} \equiv \xi^{2}, a_{10}+a_{11} \equiv \xi^{2}$ and $\gamma+\xi+a_{12}+a_{13}+a_{14}+a_{15} \equiv 0$. Therefore,

$$
\left(1+x+x^{2}+x^{3}\right)\left(t_{2}\right) \equiv 1+\pi_{K^{-}}^{3}+\pi_{K^{+}}^{1}+\pi_{K^{*}}^{5}+\pi_{K^{*}}^{7} \quad\left(\bmod \mathcal{P}_{K^{\prime}}^{8}\right)
$$

as desired.

As before, to find the first few terms of $t_{2} / t_{1}$ we let

$$
t_{2} / t_{1}=(1+\xi \pi)\left(\prod_{i=2}^{11}\left(1+b_{i} \pi^{i}\right)\right) u_{12}
$$

where $b_{i}=0$ or an odd root of unity, and $t_{12} \in U_{L_{0}}^{12}$.

Lemma 2.3.4 The unit $t_{2} / t_{1}$ may be chosen so that, modulo $\mathcal{P}_{W}^{6}$,

$$
N_{L_{0} / W_{0}}\left(t_{2} / t_{1}\right) \equiv \begin{cases}1+\pi_{W}+\pi_{W}^{2}+\pi_{W}^{4} & \text { if } \alpha=\alpha_{+} \\ 1+\pi_{W}+\pi_{W}^{2} & \text { if } \alpha=\alpha_{-}\end{cases}
$$

where $\pi w=\sqrt{30}$ is a prime element of the field $W=\mathrm{Q}_{2}(\sqrt{30})$. 
Proof We observe that, for $\alpha=\alpha_{+}$,

$$
\begin{aligned}
F\left(t_{2} / t_{1}\right) /\left(t_{2} / t_{1}\right) & =\left(F\left(t_{2}\right) / t_{2}\right)\left(t_{1} / F\left(t_{2}\right)\right) \\
& =x(\pi) / y(\pi) \\
& \equiv 1+\pi+\pi^{3}+\pi^{5}+\pi^{6}+\pi^{8}+\pi^{10}\left(\bmod \mathcal{P}_{L}^{12}\right)
\end{aligned}
$$

Now using the same method as in Lemma 2.3.2, with the help of Maple, we have the following identities, modulo 2 ,

$$
\begin{aligned}
b_{2}+b_{2}^{2}+\xi & \equiv 0 \\
b_{3}+b_{3}^{2} & \equiv 0 \\
b_{4}+b_{4}^{2}+b_{2}^{2}+b_{2}^{3}+\xi & \equiv 0 \\
b_{5}+b_{5}^{2} & \equiv 0 \\
b_{6}+b_{6}^{2}+b_{2}^{4}+b_{2}^{5} & \equiv 0 \\
b_{7}+b_{7}^{2}+b_{2}^{3}+b_{2}^{5}+b_{4}+b_{4}^{2}+b_{6}+b_{6}^{2} & \equiv 0 \\
b_{8}+b_{8}^{2}+b_{2}^{3}+b_{2} b_{4}+b_{2}^{3} b_{4} & \equiv 0 \\
b_{9}+b_{9}^{2}+b_{2}+1 & \equiv 0 \\
b_{10}+b_{10}^{2}+b_{2}^{3}+\delta+1 & \equiv 0 \\
\dot{b}_{11}^{2}+b_{11}^{2}+\delta+1 & \equiv 0
\end{aligned}
$$

The êlement $\delta$ in Equations (2.9) and (2.10), satisfies $b_{2}+b_{2}^{2}+\xi+2 \delta \equiv 0 \quad(\bmod 4)$. 
From Equations (2.2) and (2.4) we can choose $b_{3}$ and $b_{5}$ so that $b_{3}=b_{5}=0$. It follows from (2.1), (2.3), and (2.5) that, modulo 2,

$$
\begin{aligned}
& b_{2}^{2}+b_{2}^{4} \equiv\left(b_{2}+b_{2}^{2}\right)^{2} \equiv \xi^{2}, \\
& b_{4}+b_{4}^{2} \equiv b_{2}^{2}+b_{2}^{3}+\xi
\end{aligned}
$$

and

$$
b_{6}+b_{6}^{2} \equiv b_{2}^{4}+b_{2}^{5}
$$

Substituting the above identities into (2.6), we have

$$
b_{7}+b_{7}^{2} \equiv 0 \quad(\bmod 2) .
$$

Thus we can choose $b_{7}=0$. We also observe that if we let $\eta=\xi^{2}+b_{2}^{3}+b_{6}$, then, modulo 2,

$$
\begin{aligned}
\eta+\eta^{2} & \equiv 1+b_{2}^{3}+b_{2}^{4}+b_{2}^{5}+b_{2}^{6} \\
& =1+\left(b_{2}+b_{2}^{2}\right)^{3} \\
& \equiv 1+1 \equiv 0 .
\end{aligned}
$$

Multiplying $t_{2} / t_{1}$ by $1+\pi^{6}$, if necessary, we may assume that $\eta \equiv 0 \quad(\bmod 2)$. This assumption will be used in the next corollary.

It now follows from the above identities and some direct computations using Maple that, modulo $\mathcal{P}_{W_{0}}^{6}$,

$$
N_{L_{0} / W_{0}}(1+\xi \pi) \equiv 5+2 \xi+3 \pi_{W}
$$




$$
\begin{aligned}
N_{L_{0} / W_{0}}\left(1+b_{2} \pi^{2}\right) \equiv & 1+2\left(b_{2}^{2}+2 b_{2}^{3}+b_{2}^{4}\right)+ \\
& 2\left(b_{2}+3 b_{2}^{2}+2 b_{2}^{3}+2 b_{2}^{4}\right) \pi_{W} \\
N_{L_{0} / W_{0}}\left(1+b_{4} \pi^{4}\right) \equiv & 1+4\left(b_{1}+b_{4}^{4}\right)+4 b_{4} \pi_{W} \\
N_{L_{0} / W_{0}}\left(1+b_{6} \pi^{6}\right) \equiv & 1+4\left(b_{6}+b_{6}^{2}\right)+4 b_{6}^{2} \pi_{W} \\
N_{L_{0} / W_{0}}\left(1+b_{8} \pi^{8}\right) \equiv & 1 \\
N_{L_{0} / W_{0}}\left(1+b_{9} \pi^{9}\right) \equiv & 1+4 b_{9}^{2} \pi_{W} \\
N_{L_{0} / W_{0}}\left(1+b_{10} \pi^{10}\right) \equiv & 1+4 b_{10} \pi_{W} \\
N_{L_{0} / W_{0}}\left(1+b_{11} \pi^{11}\right) \equiv & 1+4 b_{11} \pi_{W}
\end{aligned}
$$

and $N_{L_{0} / W_{0}}\left(1+b_{n} \pi^{n}\right) \equiv 1$ for all $n \geq 12$, where $b_{n}=0$ or an odd root of unity (by Proposition 2.3.1(4)). Hence, by Lemma 2.2.1(4),

$$
N_{L_{0} / W_{0}}\left(t_{2} / t_{1}\right) \equiv 1+\pi_{W}+A \pi_{W}^{2}+B \pi_{W}^{3}+C \pi_{W}^{4}+D \pi_{W}^{5}(\bmod )_{W}^{\mathcal{P}_{W}^{6}}
$$

where $A=\xi+b_{2}^{2}+b_{2}^{4}, B=a_{2}+a_{2}^{4}+1, C=b_{2}^{3}+b_{4}+b_{4}^{4}+b_{6}+b_{6}^{2}+\xi^{2}$ and $D=a_{2}^{3}+a_{1}^{4}+a_{6}+a_{9}^{2}+a_{10}+a_{11}+\delta+1$.

Now since $b_{4}+b_{4}^{2} \equiv b_{2}^{2}+b_{2}^{3}+\xi(\bmod 2)$ and $b_{4}^{2}+b_{4}^{4} \equiv b_{2}^{4}+b_{2}^{6}+\xi^{2}(\bmod 2)$, we have

$$
b_{4}+b_{4}^{4} \equiv 1+b_{2}^{2}+b_{2}^{3}+b_{2}^{4}+b_{2}^{6}(\bmod 2)
$$


From (2.1), we have $b_{2}^{2}+b_{2}^{4} \equiv \xi^{2}(\bmod 2)$ and

$$
1 \equiv\left(b_{2}+b_{2}^{2}\right)^{3} \equiv b_{2}^{3}+b_{2}^{4}+b_{2}^{5}+b_{2}^{6}(\bmod 2) .
$$

Putting (2.11) into the expression of $C$ and using the above facts, we obtain $C \equiv b_{2}^{3}$ (mod 2). Using identity (2.1), we have, modulo 4,

$$
\begin{aligned}
\xi+b_{2}^{2}+b_{2}^{4} & \equiv \xi+\xi^{2}+2 b_{2}^{3} \\
& \equiv-1+2 b_{2}^{3} .
\end{aligned}
$$

Thus, modulo $\mathcal{P}_{W}^{6}$,

$$
A \pi_{W}^{2} \equiv\left(-1+2 b_{2}^{3}\right) \pi_{W}^{4} \equiv \pi_{W}^{2}+\left(1+b_{2}^{3}\right) \pi_{W}^{4}
$$

From the identity $b_{2}+b_{2}^{2} \equiv-\xi+2 \delta \quad(\bmod 4)$, we have $b_{2}^{2}+b_{2}^{4} \equiv \xi^{2}+2 b_{2}^{3} \quad(\bmod 4)$. Hence

$$
b_{2}+b_{4}^{2} \equiv 1+2\left(1+\xi+\delta+b_{2}^{2}+b_{3}^{2}\right)
$$

It follows that, modulo $\mathcal{P}_{L}^{6}$,

$$
\begin{aligned}
B \pi_{W}^{3} & \equiv\left(b_{2}+b_{2}^{4}+1\right) \pi_{W}^{3} \\
& \equiv\left(b_{2}^{2}+b_{3}^{2}+\xi+\delta\right) \pi_{W}^{5} .
\end{aligned}
$$

Therefore, with this choice of $t_{2} / t_{1}$ and $\alpha=\alpha_{+}$,

$$
\begin{aligned}
N_{L_{0} / W_{0}}\left(t_{2} / t_{1}\right) & \equiv 1+\pi_{W}+A \pi_{W}^{2}+B \pi_{W}^{3}+C \pi_{W}^{4}+D \pi_{W}^{5}\left(\bmod \mathcal{P}_{W}^{5}\right) \\
& \equiv 1+\pi_{W}+\pi_{W}^{2}+\pi_{W}^{4}+D_{2} \pi_{W}^{5},
\end{aligned}
$$


where $D_{2}=1+b_{2}^{3}+b_{4}^{4}+b_{6}+b_{9}^{2}+b_{10}+b_{11}+\delta$. Since $D_{2} \equiv 0$ or $1 \quad(\bmod 2)$ we may assume that $D_{2} \equiv 0 \quad(\bmod 2)$ by multiplying $t_{1}$ by $1+\pi^{11}$ if necessary.

In the case $\alpha=\alpha_{-}$, modulo $\mathcal{P}_{L}^{12}$,

$$
x(\pi) / y(\pi) \equiv 1+\pi+\pi^{3}+\pi^{5}+\pi^{6}+\pi^{7}+\pi^{11}
$$

Hence, $b_{\uparrow}+b_{7}^{2} \equiv 1 \quad(\bmod 2)$, and we may choose $b_{7}=\xi$. For simplicity, we assume $b_{5}=1$ instead of 0. In this case, we have, modulo $\mathcal{P}_{W_{0}}^{6}$,

$$
\begin{aligned}
& N_{L_{0} / W_{0}}(1+\xi \pi) \equiv 1+2 \xi+5 \pi_{W} \\
& N_{L_{0} / W_{0}}\left(1+b_{2} \pi^{2}\right) \equiv 1+2\left(b_{2}^{2}+2 b_{3}^{3}+b_{2}^{4}\right)+ \\
& 2\left(3 b_{2}+b_{2}^{2}+2 b_{2}^{3}+2 b_{2}^{4}\right) \pi_{W} \\
& N_{L_{0} / W_{0}}\left(1+b_{4} \pi^{4}\right) \equiv 1+4\left(b_{4}+b_{4}^{4}\right)+4 b_{4} \pi_{W} \\
& N_{L_{0} / W_{0}}\left(1+\pi^{5}\right) \equiv 1+2 \pi_{W} \\
& N_{L_{0} / W_{0}}\left(1+b_{6} \pi^{6}\right) \equiv 1+4\left(b_{6}+b_{6}^{2}\right)+4 b_{6}^{2} \pi_{W} \\
& N_{L_{0} / W_{0}}\left(1+\xi \pi^{7}\right) \equiv 5+4 \pi_{W}+4 \xi \pi W \\
& N_{L_{0} / W_{0}}\left(1+b_{8} \pi^{8}\right) \equiv 1 \\
& N_{L_{0} / W_{0}}\left(1+b_{9} \pi^{9}\right) \equiv 1+4 b_{9}^{2} \pi_{W} \\
& N_{L_{0} / W_{0}}\left(1+b_{10} \pi^{10}\right) \equiv 1+4 b_{10} \pi_{W} \\
& N_{L_{0} / W_{0}}\left(1+b_{11} \pi^{11}\right) \equiv 1+4 b_{11} \pi_{W}
\end{aligned}
$$


and $N_{L_{0} / W_{0}}\left(1+b_{n} \pi^{n}\right) \equiv 1$ for all $n \geq 12$ (by Proposition 2.3.1(4)). As in the case $\alpha=\alpha_{+}$, multiplying all of the above and assuming, without loss of generality that

$$
D_{3}=\xi+b_{2}^{3}+b_{4}^{4}+b_{6}+b_{9}^{2}+b_{10}+b_{11}+\delta \equiv 0(\bmod 2),
$$

we obtain, modulo $\mathcal{P}_{L}^{6}$,

$$
N_{L_{0} / w_{0}}\left(t_{2} / t_{1}\right) \equiv 1+\pi_{W}+\pi_{W}^{2}
$$

as desired.

Corollary 2.3.5 With the units $t_{2}$ and $t_{2} / t_{1}$ chosen as in Lemmas 2.3.2 and 2.3.4, $\operatorname{modulo} \mathcal{P}_{V}^{6}$,

$$
N_{L_{0} / V_{0}}\left(t_{1}\right) \equiv \begin{cases}1+\pi_{V}+\pi_{V}^{2}+\pi_{V}^{3}+\pi_{V}^{4}+\pi_{V}^{5} & \text { if } \alpha=\alpha_{+} \\ 1+\pi_{V}+\pi_{V}^{2}+\pi_{V}^{4} & \text { if } \alpha=\alpha_{-} .\end{cases}
$$

where $\pi_{V}=\sqrt{10}$ is a prime of the field $V=Q_{2}(\sqrt{10})$.

Proof We use the fact that $N_{L_{0} / V_{0}}\left(t_{1}\right)=N_{L_{0} / V_{0}}\left(t_{2}\right) / N_{L_{0} / V_{0}}\left(t_{2} / t_{1}\right)$ to prove the corollary.

For the case $\alpha=\alpha_{+}$, we have the following, modulo $\mathcal{P}_{V_{0}}^{6}$,

$$
\begin{aligned}
& N_{L_{0} / V_{0}}\left(1+\xi \pi^{3}\right) \equiv 3+2 \xi+2 \pi_{V}+6 \xi \pi_{V} \\
& N_{L_{0} / V_{0}}\left(1+\xi \pi^{4}\right) \equiv 1+4 \xi \pi V
\end{aligned}
$$




$$
\begin{aligned}
N_{L_{0} / V_{0}}\left(1+\xi \pi^{5}\right) & \equiv 5+6 \pi_{V}+6 \xi \pi_{V} \\
N_{L_{0} / V_{0}}\left(1+a_{6} \pi^{6}\right) & \equiv 1+4\left(a_{6}+a_{6}^{2}\right)+4\left(a_{6}+a_{6}^{2}\right) \pi_{V} \\
N_{L_{0} / V_{0}}\left(1+\xi \pi^{7}\right) & \equiv 5+4 \xi \pi_{V} \\
N_{L_{0} / V_{0}}\left(1+a_{8} \pi^{8}\right) & \equiv 1 \\
N_{L_{0} / V_{0}}\left(1+a_{10} \pi^{10}\right) & \equiv 1+4 a_{10} \pi V \\
N_{L_{0} / V_{0}}\left(1+a_{11} \pi^{11}\right) & \equiv 1+4 a_{11} \pi_{V}
\end{aligned}
$$

and $N_{L_{0} / v_{0}}\left(1+a_{n} \pi^{n}\right) \equiv 1$ for all $n \geq 12$ (by Proposition 2.3.1(3)).

In the case $\alpha=\alpha_{-}$, the only two differences are, modulo $\mathcal{P}_{V_{0}}^{6}$,

$$
N_{L_{0} / V_{0}}\left(1+\xi \pi^{3}\right) \equiv 3+2 \xi+2 \xi \pi_{V}+6 \pi_{V}
$$

and

$$
N_{L_{0} / V_{0}}\left(1+\xi \pi^{5}\right) \equiv 5+2 \pi_{V}+2 \xi \pi_{V}
$$

In both cases, we have, modulo $\mathcal{P}_{V_{0}}^{6}$,

$$
N_{L_{0} / V_{0}}\left(t_{2}\right) \equiv 1+\xi^{2} \pi_{V}+\left(\xi^{2}+a_{6}+a_{6}^{2}\right) \pi_{V}^{4}+\left(a_{6}+a_{6}^{2}+a_{10}+a_{11}\right) \pi_{V}^{5}
$$

Since $a_{6}+a_{6}^{2} \equiv \xi^{2} \quad(\bmod 2)$, and since we already chose, in the proof of Lemma 2.3.2, $a_{10}$ and $a_{11}$ so that $a_{10}+a_{11} \equiv \xi^{2} \quad(\bmod 2)$, we obtain

$$
N_{L_{0} / V_{0}}\left(t_{2}\right) \equiv 1+\xi^{2} \pi_{V}^{2} \quad\left(\bmod \mathcal{P}_{V_{0}}^{6}\right)
$$


To compute $N_{L_{0} / V_{0}}\left(t_{2} / t_{1}\right)$ for the case $\alpha=\alpha_{+}$, we observe the following, modulo $\mathcal{P}_{V_{0}}^{6}$

$$
\begin{aligned}
N_{L_{0} / V_{0}}(1+\xi \pi) & \equiv 5+3 \pi_{V}+6 \xi \pi_{V} \\
N_{L_{0} / V_{0}}\left(1+b_{2} \pi^{2}\right) & \equiv 1+2\left(b_{2}^{2}+b_{2}^{4}\right)+2\left(b_{2}+b_{2}^{2}\right) \pi_{V}+4 b_{2}^{3}, \\
N_{L_{0} / V_{0}}\left(1+b_{4} \pi^{4}\right) & \equiv 1+4\left(b_{4}+b_{4}^{4}\right)+4 b_{4} \pi_{V} \\
N_{L_{0} / V_{0}}\left(1+b_{6} \pi^{6}\right) & \equiv 1+4\left(b_{6}+b_{6}^{2}\right)+4\left(b_{6}+b_{6}^{2}\right) \pi_{V} \\
N_{L_{0} / V_{0}}\left(1+b_{8} \pi^{8}\right) & \equiv 1 \\
N_{L_{0} / V_{0}}\left(1+b_{9} \pi^{9}\right) & \equiv 1+4 b_{9}^{2} \pi_{V} \\
N_{L_{0} / V_{0}}\left(1+b_{10} \pi^{10}\right) & \equiv 1+4 b_{10} \pi_{V} \\
N_{L_{0} / V_{0}}\left(1+b_{11} \pi^{11}\right) & \equiv 1+4 b_{11} \pi_{V}
\end{aligned}
$$

and $N_{L_{0} / V_{0}}\left(1+b_{n} \pi^{n}\right) \equiv 1$ for all $n \geq 12$ (by Proposition 2.3.1(3)).

In the case $\alpha=\alpha_{-}$, recall that we chose $b_{5}=1$ and $b_{7}=\xi$, and hence have the following changes, modulo $\mathcal{P}_{V_{0}}^{6}$,

$$
\begin{aligned}
N_{L_{0} / V_{0}}(1+\xi \pi) & \equiv 1+5 \pi_{V}+6 \xi \pi_{V} \\
N_{L_{0} / V_{0}}\left(1+b_{2} \pi^{2}\right) & \equiv 1+2\left(b_{2}^{2}+b_{2}^{4}\right)+6\left(b_{2}+b_{2}^{2}\right) \pi_{V}+4 b_{2}^{3} \\
N_{L_{0} / V_{0}}\left(1+\pi^{5}\right) & \equiv 1+6 \pi V
\end{aligned}
$$

and $N_{L_{0} / V_{0}}\left(1+\xi \pi^{7}\right) \equiv 5+4 \xi \pi v$. Using the above facts, we have the following, 
modulo $\mathcal{P}_{V_{0}}^{6}$

$$
N_{L_{0} / V_{0}}\left(t_{2} / t_{1}\right) \equiv \begin{cases}1+\pi_{V}+\xi^{2} \pi_{V}^{2}+\xi \pi_{V}^{3}+M_{4} \pi_{V}^{4}+M_{5} \pi_{V}^{5} & \text { if } \alpha=\alpha_{+} \\ 1+\pi_{V}+\xi^{2} \pi_{V}^{2}+\xi^{2} \pi_{V}^{3}+N_{4} \pi_{V}^{4}+N_{5} \pi^{5} & \text { if } \alpha=\alpha_{-},\end{cases}
$$

where, modulo 2,

$$
\begin{aligned}
& M_{4}=N_{4} \equiv 1+b_{2}+b_{2}^{2}+b_{4}+b_{4}^{4}+b_{6}+b_{6}^{2}, \\
& M_{5} \equiv \xi+b_{4}^{4}+b_{9}^{2}+b_{10}+b_{11}+\delta, \\
& N_{5} \equiv 1+b_{4}^{4}+b_{9}^{2}+b_{10}+b_{11}+\delta
\end{aligned}
$$

and $2 \delta \equiv b_{2}+b_{2}^{2}+\xi \quad(\bmod 4)$, defined as in the proof of Lemma 2.3.4. It follows from the computation of $B$ (in the proof of the previous lemma) that $b_{4}+b_{4}^{4}+b_{6}+b_{6}^{2} \equiv \xi^{2}$

(mod 2). Together with Equation (2.1), we have $M_{4} \equiv N_{4} \equiv 0 \quad(\bmod 2)$. With $D_{2}, D_{3}$ and $\eta$ defined as in the proof of Lemma 2.3.4, we have $M_{5} \equiv D_{2}+\eta \equiv 0$ $(\bmod 2)$ and $N_{5} \equiv D_{3}+\eta \equiv 0(\bmod 2)$. Hence, modulo $\mathcal{P}_{V_{0}}^{6}$,

$$
N_{L_{0} / V_{0}}\left(t_{2} / t_{1}\right) \equiv \begin{cases}1+\pi_{V}+\xi^{2} \pi_{V}^{2}+\xi \pi_{V}^{3} & \text { if } \alpha=\alpha_{+} \\ 1+\pi_{V}+\xi^{2} \pi_{V}^{2}+\xi^{2} \pi_{V}^{3} & \text { if } \alpha=\alpha_{-} .\end{cases}
$$

By Lemma 2.2.1(3), $N_{L_{0} / V_{0}}\left(t_{1}\right)=N_{L_{0} / V_{0}}\left(t_{2}\right) / N_{L_{0} / V_{0}}\left(t_{2} / t_{1}\right) \in V$, we therefore have for the case $\alpha=\alpha_{+}$, modulo $\mathcal{P}_{V}^{6}$,

$$
\begin{aligned}
N_{L_{0} / V_{0}}\left(t_{1}\right) & \equiv \frac{1+\xi^{2} \pi_{V}^{2}}{1+\pi_{V}+\xi^{2} \pi_{V}^{2}+\xi \pi_{V}^{3}} \\
& \equiv 1+\pi_{V}+\pi_{V}^{2}+\pi_{V}^{3}+\pi_{V}^{4}+\pi_{V}^{5} .
\end{aligned}
$$


Similarly, in the case $\alpha=\alpha_{-}$, modulo $\mathcal{P}_{V}^{6}$,

$$
N_{L_{0} / V_{0}}\left(t_{1}\right) \equiv 1+\pi_{V}+\pi_{V}^{2}+\pi_{V}^{4}
$$

as desired.

More details on the method of computations of the following result can be found in the proof of Proposition 3.4.5.

Corollary 2.3.6 With $c_{1}, c_{2}$ and the map $k$ defined as in Section 2.2,

$$
k\left(\left(1-x^{2}\right)\left(c_{1}-c_{2}\right)\right) \equiv 1+\pi^{8}+\pi^{13}+\pi^{16}\left(\bmod \mathcal{P}_{L}^{18}\right)
$$

Proof We observe that

$$
\begin{aligned}
k\left(\left(1-x^{2}\right)\left(c_{1}-c_{2}\right)\right) & =\left(1-x^{2}\right) k\left(\left((x-x y) b-(x+y) b^{\prime}\right)\right) \\
& =\left(1-x^{2}\right)(x-x y)\left(t_{2}\right) \cdot\left(x^{2}-1\right)(x+y)\left(\pi i_{1}\right)
\end{aligned}
$$

With the choice of $t_{2}$ and $t_{2} / t_{1}$ as in Lemmas 1.3.2 and 1.3.4, and using the identity $t_{1}=t_{2} /\left(t_{2} / t_{1}\right)$, we have, modulo $\mathcal{P}_{L}^{18}$,

$$
\left(x^{2}-1\right)(x+y)\left(\pi t_{1}\right) \equiv 1+\pi^{8}+\pi^{13}+\xi^{2} \pi^{14}+\xi^{2} \pi^{15}+\xi^{2} \pi^{16}+\xi \pi^{17}
$$

and

$$
\left(1-x^{2}\right)(x-x y)\left(t_{2}\right) \equiv 1+\xi^{2} \pi^{14}+\xi^{2} \pi^{15}+\xi \pi^{16}+\xi \pi^{17} .
$$


Multiplying the above results, we obtain

$$
k\left(1-x^{2}\right)\left(c_{1}-c_{2}\right) \equiv 1+\pi^{8}+\pi^{13}+\pi^{16}\left(\bmod \mathcal{P}_{L}^{18}\right),
$$

as desired.

Before proving the injectivity of the map $k$, we state and prove the following corollary.

Corollary 2.3.7 With the elements $\tau, \lambda$, and $\rho$ defined as in the beginning of this section

$$
\begin{aligned}
& \tau\left(t_{2}\right) \equiv 25+12 \sqrt{3} \quad\left(\bmod \mathcal{P}_{\bar{K}}^{10}\right), \\
& \lambda\left(\pi t_{1}\right) \equiv 3+2 \sqrt{10} \quad\left(\bmod \mathcal{P}_{V}^{8}\right),
\end{aligned}
$$

and

$$
\rho\left(t_{2} /\left(\pi t_{1}\right)\right) \equiv 15+4 \sqrt{30}\left(\bmod \mathcal{P}_{W}^{10}\right)
$$

Proof We observe that, for $a, u \in U_{K}^{1}$, modulo $\mathcal{P}_{K}^{10}$,

$$
\begin{aligned}
(1-y)\left(1+a \pi_{K}^{3}+u \pi_{K}^{8}\right)= & \frac{1+a \pi_{K}^{3}+u \pi_{K}^{8}}{1+y\left(a \pi_{K}^{3}\right)+y\left(u \pi_{K}^{8}\right)} \\
\equiv & \left(1+a \pi_{K}^{3}+u \pi_{K}^{8}\right)\left(1-y\left(a \pi_{K}^{3}\right)-y\left(u \pi_{K}^{8}\right)\right. \\
& \left.+y\left(a \pi_{K}^{3}\right)^{2}+y\left(a \pi_{K}^{3}\right)^{3}\right) \\
\equiv & (1-y)\left(1+a \pi_{K}^{3}\right)+\left(u \pi_{K}^{8}-y\left(u \pi_{K}^{8}\right)\right) .
\end{aligned}
$$


By letting $u=u_{0}+u_{1} \pi_{K}+\cdots$, where $u_{i}$ is either 0 or 1 , it is straightforward that $u \pi_{K}^{8}-y\left(u \pi_{K}^{8}\right) \equiv 0 \quad\left(\bmod \mathcal{P}_{K}^{10}\right)$. Thus

$$
(1-y)\left(1+a \pi_{K}^{3}+u \pi_{K}^{8}\right) \equiv(1-y)\left(1+a \pi_{K}^{3}\right) \quad\left(\bmod \mathcal{P}_{K}^{10}\right)
$$

It now follows from Corollary 2.3.3 and the definition of $\tau$ that if we let $\pi_{K}^{\prime}=y\left(\pi_{K}\right)=$ $1-\sqrt{3}$, then

$$
\begin{aligned}
\tau\left(t_{2}\right) & \equiv(1-y)\left(1+\pi_{K}^{3}+\pi_{K}^{4}+\pi_{K}^{5}+\pi_{K}^{7}\right) \quad\left(\bmod \mathcal{P}_{K}^{10}\right) \\
& \equiv \frac{1+\pi_{K}^{3}+\pi_{K}^{4}+\pi_{K}^{5}+\pi_{K}^{7}}{1+\left(\pi_{K}^{1}\right)^{3}+\left(\pi_{K}^{1}\right)^{4}+\left(\pi_{K}^{1}\right)^{5}+\left(\pi_{K}^{1}\right)^{7}}\left(\bmod \mathcal{P}_{K}^{10}\right) \\
& \equiv 25+12 \sqrt{3}\left(\bmod \mathcal{P}_{K}^{10}\right) .
\end{aligned}
$$

This completed the proof of the first statement. To prove the second statement in the case $\alpha=\alpha_{+}$, we use the same arguments together with the results of Corollary 2.3.5, the fact that $N_{L / V}(\pi)=-16 / 5-3 \sqrt{10} / 5$, and consider, modulo $\mathcal{P}_{V}^{8}$,

$$
\begin{aligned}
\lambda\left(\pi t_{1}\right) & =\lambda\left(t_{1}\right) \cdot(1-x)\left(1+y+y^{2}+y^{3}\right)(\pi) \\
& \equiv \frac{1+\pi_{V}+\pi_{V}^{2}+\pi_{V}^{3}+\pi_{V}^{4}+\pi_{V}^{5}}{1-\pi_{V}+\pi_{V}^{2}-\pi_{V}^{3}+\pi_{V}^{4}-\pi_{V}^{5}} \cdot(1-x)\left(-\frac{16}{5}-\frac{3 \sqrt{10}}{5}\right) \\
& \equiv \frac{111+111 \sqrt{10}}{111-111 \sqrt{10}} \cdot \frac{16+3 \sqrt{10}}{16-3 \sqrt{10}} \\
& \equiv \frac{(111+111 \sqrt{10})^{2}}{111^{2}-111^{2} \cdot 10} \cdot \frac{(16+3 \sqrt{10})^{2}}{256-90} \\
& \equiv 3+2 \sqrt{10}
\end{aligned}
$$


Since $N_{L / W}\left(1+\pi^{8}\right) \equiv 1+\pi_{W}^{6} \quad\left(\bmod \mathcal{P}_{W}^{8}\right), N_{L / W}\left(1+\pi^{18}\right) \equiv 1+\pi_{W}^{7} \quad\left(\bmod \mathcal{P}_{W}^{8}\right)$ and $N_{L / W}\left(1+\pi^{8}\right) \equiv 1 \quad\left(\bmod \mathcal{P}_{V}^{6}\right)$, we may multiply $t_{2} / t_{I}$ by $1+\pi^{8}$ and/or $1+\pi^{18}$, if necessary, without affecting the results of Corollary 2.3.5, to assume the results of Lemma 2.3.4 modulo $\mathcal{P}_{W}^{8}$. Hence in the case $\alpha=\alpha_{+}$, to prove the third statement we use Lemma 2.3.4 and the fact that $N_{L / W}(\pi)=-14 / 5-\sqrt{30} / 5$ and observe that

$$
\begin{aligned}
\rho\left(t_{2} /\left(\pi t_{1}\right)\right) & \equiv \rho\left(t_{2} / t_{1}\right) \cdot(x-1)\left(1+x y+x^{2}+(x y)^{3}\right)(\pi) \\
& \equiv \frac{1+\pi_{W}+\pi_{W}^{2}+\pi_{W}^{4}}{1-\pi_{W}+\pi_{W}^{2}+\pi_{W}^{4}} \cdot \frac{14-\sqrt{30}}{14+\sqrt{30}} \\
& \equiv \frac{931+\sqrt{30}}{931-\sqrt{30}} \cdot \frac{14-\sqrt{30}}{14+\sqrt{30}} \\
& \equiv \frac{(931+\sqrt{30})^{2}}{931^{2}-30} \cdot \frac{(14-\sqrt{30})^{2}}{196-30} \\
& \equiv 15+4 \sqrt{30} .
\end{aligned}
$$

In the case $\alpha=\alpha_{-}$, we have

$$
\left(1+y+y^{2}+y^{3}\right)(\pi)=4 / 5-\sqrt{10} / 5
$$

and

$$
\left(1+x y+x^{2}+(x y)^{3}\right)(\pi)=6 / 5+\sqrt{30} / 5
$$

The proofs of the last two statements for this case can also be carried out in the same manner.

We are now in a position to prove the main result of this chapter. 
Theorem 2.3.8 The homomorphism $k$ is injective.

Proof From the formulae of Section 1.2

$$
j\left(c_{1}\right)=t_{2} x\left(t_{2}\right) y\left(\pi t_{1}\right)^{-1}\left(\pi t_{1}\right)^{-1}
$$

and

$$
j\left(c_{2}\right)=t_{2} x y\left(t_{2}\right) x\left(\pi t_{1}\right)\left(\pi t_{1}\right)^{-1},
$$

we see that $v_{L}\left(j\left(c_{1}\right)\right)=-2$ and $v_{L}\left(j\left(c_{2}\right)\right)=0$ so that the homomorphism

$$
v_{L} \circ k: \operatorname{Ker}(d) \longrightarrow L^{*} \longrightarrow 2 \mathrm{Z}
$$

is onto. Therefore to prove $k$ is injective, it suffices to show that

$$
k: \operatorname{Ker}(d) \cap k^{-1}\left(\mathcal{O}_{L}^{*}\right) \rightarrow \mathcal{O}_{L}^{*}
$$

is injective. As in the proof of Snaith [32], Theorem 6.1.7, to prove the injectivity of $k$, it suffices to show that $\sigma\left(t_{2}^{2}\right), \tau\left(t_{2}^{2}\right), \lambda\left(\left(\pi t_{1}\right)^{2}\right), \rho\left(\left(t_{2} /\left(\pi t_{1}\right)\right)^{2}\right)$, and $k\left(1-x^{2}\right)\left(c_{1}-c_{2}\right)$ have infinite order in $\mathcal{O}_{L}^{*}$ where $\sigma, \tau, \lambda$ and $\rho$ are defined as in the begining of Section 2.3, and $c_{1}, c_{2}$ are defined as in Section 2.2. The desired result now follows from Lemma 2.3.2 and Corollaries 2.3.6 and 2.3.7. 


\section{Chapter 3}

\section{The Second Chinburg Conjecture}

\subsection{The Main Theorem}

In this section we shall study the Chinburg invariant $\Omega(N / Q, 2)$ of Section 1.5 in the case when $N / Q$ is a Galois extension with $\operatorname{Gal}(N / \mathrm{Q}) \cong Q_{8}$ whose 2-adic completion is one of the $Q_{8}$-extensions, $L_{ \pm} / Q_{2}$ of Section 2.1.

Suppose that $Y$ is a finitely generated $\mathrm{Z}[G]$-module which has no $\mathrm{Z}$-torsion, where $G$ is a finite group. In this case the universal coefficient theorems for homology and cohomology (Spanier [36], page 222 and page 236) imply that, for $i \geq 0$,

$$
H_{\mathrm{i}}(G ; Y) \otimes_{\mathrm{z}} \mathbf{Z}_{2} \cong H_{\mathrm{i}}\left(G ; Y \otimes_{\mathrm{z}} \mathbf{Z}_{2}\right)
$$
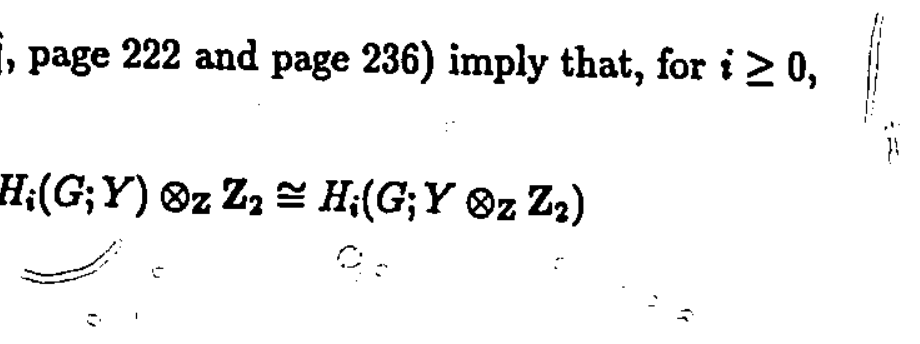
and

$$
H^{i}(G ; Y) \otimes_{\mathrm{Z}} \mathbf{Z}_{2} \cong H^{i}\left(G ; Y \otimes_{\mathrm{z}} \mathbf{Z}_{2}\right)
$$

Consequently, even for $i=0,-1$,

$$
\hat{H}^{\mathrm{i}}(G ; Y) \otimes_{\mathrm{Z}} \mathbf{Z}_{2} \cong \hat{H}^{\mathrm{i}}\left(G ; Y \otimes_{\mathrm{z}} \mathbf{Z}_{2}\right)
$$

In particular, if $G$ is a 2-group then each $\hat{H}^{i}(G ; Y)$ is a finite 2-group and under the identification $a \otimes\left(\sum_{j \geq m} a_{j} 2^{j}\right) \sim a^{\sum_{j \geq m} a_{j} 2^{j}}$, where $a \in G, m \in Z$, and $a_{j}=0$ or 1, we have

for all $i \in \mathbf{Z}$.

$$
\hat{H}^{i}(G ; Y) \cong \hat{H}^{i}(G ; Y) \otimes_{\mathrm{z}} \mathrm{Z}_{2} \cong \hat{H}^{i}\left(G ; Y \otimes_{\mathrm{z}} Z_{2}\right)
$$

Let $\bar{Y}$ denote the 2-ailic closure of $Y$. Let $L=L_{ \pm}$and $k: \operatorname{Ker}(d) \rightarrow L^{*}$ be as in Theorem 2.3.9. For abbreviation, set

$$
Y_{L}=\operatorname{Ker}(d) \cap k^{-1}\left(\mathcal{O}_{L}^{*}\right)
$$

The commutative diagram of Section 2.2 implies that

$$
k_{*}: \hat{H}^{*}(G ; \operatorname{Ker}(d)) \stackrel{\Xi}{\longrightarrow} \hat{H}^{*}\left(G ; L^{*}\right)
$$

is an isomorphism for all $G \leq Q_{8}$. Hence

$$
k_{\star}: \hat{H}^{*}\left(G ; Y_{L}\right) \stackrel{\cong}{\longrightarrow} \hat{H}^{*}\left(G ; \mathcal{O}_{L}^{*}\right)
$$


is also an isomorphism for all $G \leq Q_{8}$. Since

$$
\bar{Y}_{L}=Y_{L} \otimes \mathbf{z} \mathbf{Z}_{2}
$$

we see that

$$
k_{*}: \hat{H}^{*}\left(G ; \bar{Y}_{L}\right) \stackrel{\cong}{\longrightarrow} \hat{H}^{*}\left(G ; \mathcal{O}_{L}^{*}\right)
$$

is also an isomorphism for all $G \leq Q_{8}$.

By Theorem 2.3.9 we have an injection of the form

$$
k: \bar{Y}_{L}=\overline{\operatorname{Ker}(d) \cap \overline{k^{-1}\left(\mathcal{O}_{L}^{*}\right)}} \longrightarrow \mathcal{O}_{L}^{*}
$$

which must have a finite cokernel, $M$, since $\mathcal{O}_{L}^{*}$ contains a copy of $\mathbf{Z}_{2}\left[Q_{8}\right]$ of finite index. Hence $M$ is a finite 2-group. It follows from the long exact (cohomology) sequence associated with

$$
0 \rightarrow \overline{Y_{L}} \rightarrow \mathcal{O}_{L}^{*} \rightarrow M \longrightarrow 0
$$

and the fact that $k: \overline{Y_{L}} \longrightarrow \mathcal{O}_{L}$ is a cohomological equivalence, that $M$ is a cohomologically trivial $\mathrm{Z}\left[Q_{8}\right]$-module.

Recall that the residue field $\bar{L}$ has order two, that there is an isomorphism of the form

$$
\mathcal{O}_{\Sigma}^{*} /\left(k\left(\bar{Y}_{L}\right)\right) \cong L^{*} /(\overline{k(\overline{K e r}(d)))}
$$


and that there is an exact sequence of the form

$$
0 \longrightarrow \bar{Y}_{L} \longrightarrow \overline{k(\operatorname{Ker}(d))} \longrightarrow \mathrm{Z} \rightarrow 0
$$

The fact that $L^{*} \cong \mathcal{O}_{L}^{*} \oplus \mathrm{Z}$ implies that as an abelian group,

$$
K \operatorname{er}(d) \cong Y_{L} \oplus \mathbf{Z}
$$

With this identification the commutative diagram of Section 2.2 becomes

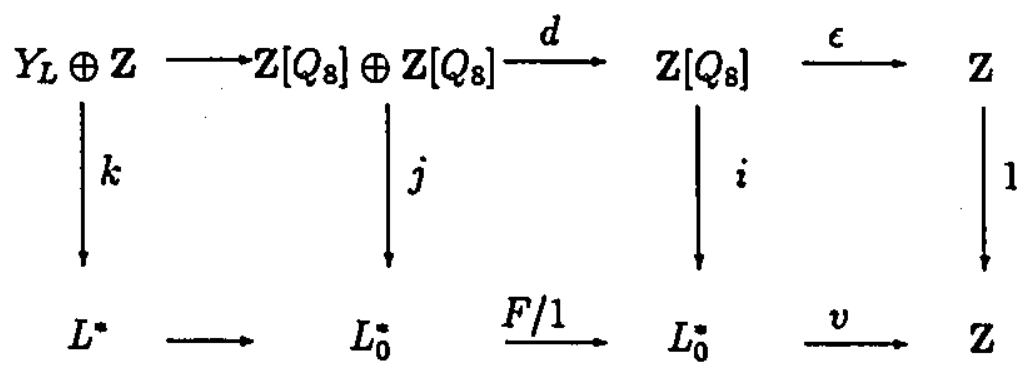

By pushing out the upper 2-extension along $k$, we obtain an internediate diagram of the following form, in which $j^{\prime}$ and $k$ are injective.

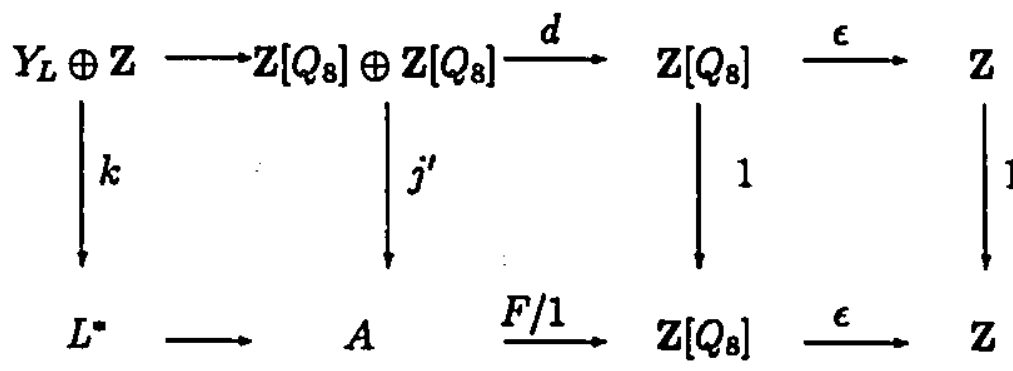


In the above diagram,

$$
A=\left(L^{*} \oplus \mathbf{Z}\left[Q_{8}\right] \oplus \mathbf{Z}\left[Q_{8}\right]\right) / S
$$

where $S$ is the subgroup of $L^{*} \oplus \mathrm{Z}\left[Q_{8}\right] \oplus \mathrm{Z}\left[Q_{8}\right]$ consisting of all $(k(a),-a)$ where $a \in Y_{L} \oplus Z$. The map $j^{\prime}$ is defined by $j^{\prime}(b, c)=(0, b, c)+S$.

If $X$ and $X_{2}$ are as in Section 1.5, then

$$
\Omega\left(L / Q_{2}, 1+X_{2}\right)=\left[A /\left(1+X_{2}\right)\right] \in \mathcal{C L}\left(Z\left[Q_{8}\right]\right)
$$

and

$$
\Omega(N / \mathrm{Q}, 2)=[X]+\left[A /\left(1+X_{2}\right)\right] \in \mathcal{C L}\left(\mathrm{Z}\left[Q_{8}\right]\right)
$$

We also have an induced commutative diagram of exact sequences of the following form.

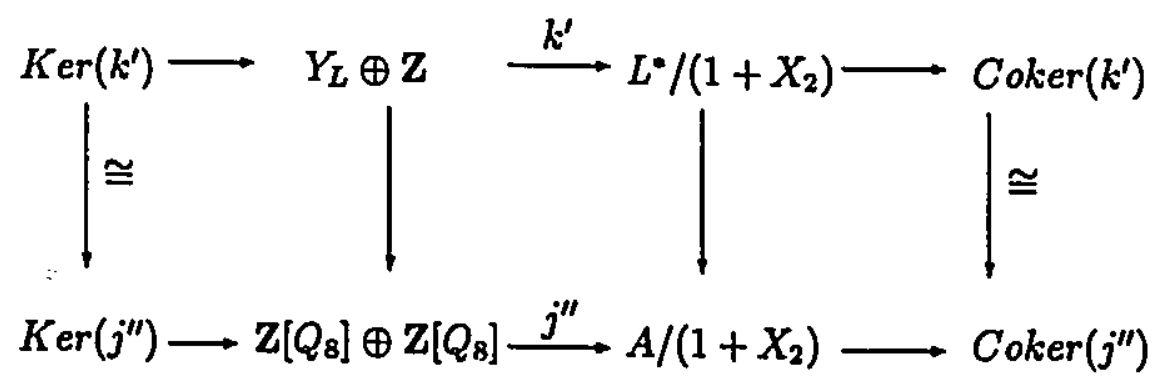


Therefore, in $\mathcal{C L}\left(\mathrm{Z}\left[Q_{8}\right]\right)$,

$$
\begin{aligned}
{\left[A /\left(1+X_{2}\right)\right] } & =\left[\operatorname{Coker}\left(j^{\prime \prime}\right)\right]+\left[\mathrm{Z}\left[Q_{8}\right] \oplus \mathrm{Z}\left[Q_{8}\right]\right]-\left[\operatorname{Ker}\left(j^{\prime \prime}\right)\right] \\
& =\left[\operatorname{Coker}\left(j^{\prime \prime}\right)\right]-\left[\operatorname{Ker}\left(j^{\prime \prime}\right)\right] \\
& =\left[\operatorname{Coker}\left(k^{\prime}\right)\right]-\left[\operatorname{Ker}\left(k^{\prime}\right)\right]
\end{aligned}
$$

and

$$
\Omega(N / \mathrm{Q}, 2)=\left[X^{\prime}\right]+\left[\operatorname{Coker}\left(k^{\prime}\right)\right]-\left[\ldots \operatorname{Cr}\left(k^{\prime}\right)\right]
$$

If we choose $X_{2} \subset 8 \mathcal{O}_{L}$ then the homomorphism

$$
1+X_{2} \longrightarrow L^{*} \longrightarrow L^{*} /\left(k\left(\left(Y_{L} \otimes Z_{2}\right) \oplus \mathbf{Z}\right)\right) \cong M
$$

will be zero, by the calculations which will be culminated in Section 3.2. Therefore

$$
\begin{aligned}
\operatorname{Coker}\left(k^{\prime}\right) & \cong\left(L^{*} /\left(1+X_{2}\right)\right) /\left(k^{\prime}\left(Y_{L} \oplus \mathbf{Z}\right)\right) \\
& \cong\left(L^{*} /\left(1+X_{2}\right)\right) /\left(k\left(\left(Y_{L} \otimes \mathbf{Z}_{2}\right) \oplus \mathbf{Z}\right)\right) \\
& \cong L^{*} /\left(k\left(\left(Y_{L} \otimes \mathbf{Z}_{2}\right) \oplus \mathbf{Z}\right)\right) \\
& \cong M
\end{aligned}
$$

Combining the above results, we obtain

$$
\Omega(N / \mathrm{Q}, 2)=[X]+[M]-\left[K \operatorname{er}\left(k^{\prime}\right)\right] \in \mathcal{C L}\left(\mathrm{Z}\left[Q_{8}\right]\right)
$$

We now state and prove the main theorem. 
Theorem 3.1.1 Let $N / Q$ be a Galois extension with $\operatorname{Gal}(N / \mathrm{Q}) \cong Q_{8}$ whose 2-adic completion is isomorphic to one of the extensions, $L_{ \pm} / \mathrm{Q}_{2}$, of Section 2.1. Let $W_{N / \mathrm{Q}}$ denote the root number class of Section 1.3. Then

$$
\Omega(N / \mathrm{Q}, 2)=W_{N / Q} \in \mathcal{C L}\left(\mathbf{Z}\left[Q_{8}\right]\right) \cong\{ \pm 1\}
$$

Proof It will be shown in Section 3.2 that in $\mathcal{C L}\left(Z\left[Q_{8}\right]\right),[M]$ is trivial in both $L_{ \pm} / Q_{2}$ cases. The results of Sections 3.3 and 3.4 indicate that $[X]=-W_{N / Q}$ and $\left[\operatorname{Ker}\left(k^{\prime}\right)\right]$ is non-trivial in the $L_{+} / Q_{2}$ case, and $[X]=W_{N / Q}$ and $\left[\operatorname{Ker}\left(k^{\prime}\right)\right]$ is trivial in the $L_{-} / Q_{2}$ case. The desired result now follows from the formula preceding the statement of the theorem.

\subsection{Computation of $[M]$}

Recall that in the previous section, the cokernel $M=L^{*} /(\overline{(\operatorname{Ker}(d))})$ is a finite 2group which is a cohomologically trivial $\mathbf{Z}\left[Q_{8}\right]$-module. The purpose of this section is to show thä $[M]$ is trivial in $\mathcal{C C}\left(\mathrm{Z}\left[Q_{8}\right]\right) \cong \mathrm{Z} / 2 \cong\{ \pm 1\}$. We define

$$
M_{ \pm}=\left\{z \in M \mid x^{2}(z)= \pm z\right\}
$$

where $x \in Q_{8}$ is defined as in the beginning of Section 2.1 . 
Lemma 3.2.1 Let $M^{Q_{\mathrm{s}}}$ be the subset of elements of $M$ fixed by $Q_{\mathrm{8}}$. Then

$$
M^{Q_{B}} \cong \mathrm{Z} / 2
$$

Proof Since $M$ is cohomologically trivial $Z\left[Q_{8}\right]-$ module, from the short exact sequence

$$
0 \longrightarrow \overline{k(\operatorname{Ker}(d))} \rightarrow L^{*} \longrightarrow M \rightarrow 0
$$

we obtain an exact cohomology sequence

$$
0 \longrightarrow \overline{k(K e r(d))}^{Q_{s}} \rightarrow\left(L^{*}\right)^{Q_{s}} \rightarrow M^{Q_{s}} \rightarrow H^{1}\left(Q_{8} ; \overline{k(K \operatorname{ker}(d))}\right)=0 .
$$

Hence,

$$
M^{Q_{s}} \cong \frac{\left(L^{*}\right)^{Q_{s}}}{k(\operatorname{Ker}(d))^{Q_{s}}}=\frac{\mathrm{Q}_{2}^{*}}{\left\langle j(\sigma b), j\left(\sigma b^{\prime}\right)\right\rangle} .
$$

It follows from Lemma 2.3.2 that

$$
j(\sigma b)=\sigma\left(t_{2}\right)=5+8 a \in U_{\mathrm{Q}_{2}}^{2} \backslash U_{\mathrm{Q}_{2}}^{3}
$$

generates $U_{Q_{2}}^{2}$ for some $a \in \mathbf{Z}_{2}$. Using Theorem 2.3.7(2), we have

$$
j\left(\sigma b^{\prime}\right)=\sigma\left(\pi t_{1}\right)=\sigma(\pi)\left((1+x)\left(1+\pi_{V}+\pi_{V}^{2}+\pi_{V}^{3}+u \pi_{V}^{6}\right)\right)=2 c
$$

where $V=\mathbf{Q}_{2}(\sqrt{10}), \pi_{V}=\sqrt{10}, u \in \mathcal{O}_{V}, \pi=\pi_{L}$, and $c \in U_{\mathbf{Q}_{2}}^{2}$. Since the element $j(\sigma b)$ generates $U_{\mathrm{Q}_{2}}^{2}$, it generates c. Therefore, $2 \sim 1$ in $M^{Q_{\mathrm{a}}}$ and $M^{Q_{\mathrm{a}}} \cong \mathrm{Z} / 2$, as desired. 
By Snaith [32], page $149-151, M_{-}$is a principal left $\mathrm{H}_{\mathbf{z}}$-module, $\left|M_{-}\right| \geq 4$ and

$$
M_{-} \cong \frac{L^{*} / E^{*}}{\left(k\left(c_{1}\right), k\left(c_{2}\right)\right)}
$$

where $c_{1}$ and $c_{2}$ are the generators of $\operatorname{Ker}(d)$ defined as in Section 2.2 and $\mathrm{H}_{\mathrm{Z}}$ is the ring of integral quaternions.

To compute $M_{-}$, we prove the following proposition.

Proposition 3.2.2 The images of $c_{1}$ and $c_{2}$ under the map $k$ are given as follows:

$$
\text { (1) } k\left(c_{1}\right)=\frac{1}{\pi^{2}}\left(1+\pi+\pi^{2}+u \pi^{4}\right)
$$

and

(2) $k\left(c_{2}\right) \equiv 1+\pi^{3}+\pi^{4}\left(\bmod \mathcal{P}_{L}^{6}\right)$

for some $u \in \mathcal{O}_{L}^{*}$.

Proof It follows from the commutative diagram of Section 2.2 that

$$
k\left(c_{1}\right)=\frac{t_{2} x\left(t_{2}\right)}{t_{1} \pi y\left(t_{1} \pi\right)}: \text { and } k\left(c_{2}\right)=t_{2} x y\left(t_{2}\right) \frac{x\left(\pi t_{1}\right)}{\pi t_{1}}
$$

As in Section 2.3, for some $v, w \in U_{L_{0}}^{*}$, we have $t_{2}=1+\xi \pi^{3}+v \pi^{4}$ and $t_{1}=1+\xi \pi+w \pi^{2}$ where $\xi$ is a primitive third root of unity. We consider

$$
\begin{gathered}
t_{2} x\left(t_{2}\right)=\left(1+\xi \pi^{3}+v \pi^{4}\right)\left(1+\xi x\left(\pi^{3}\right)+x\left(v \pi^{4}\right)\right) \\
\equiv 1+\xi\left(\pi^{3}+x\left(\pi^{3}\right)\right) \quad\left(\bmod \mathcal{P}_{L_{0}}^{4}\right) . \\
\sigma=
\end{gathered}
$$


Since $x\left(\pi^{3}\right)=\pi^{3}\left(1+\pi^{3}+\pi^{4}+\cdots\right)^{3}$, we have $t_{2} x\left(t_{2}\right) \equiv 1 \quad\left(\bmod \mathcal{P}_{L_{0}}^{4}\right)$.

Using the identity $y(\pi)=\pi+\pi^{2}+\pi^{3}+\pi^{4}+\cdots$ and the fact that $w+y(w) \in \mathcal{P}_{L_{0}}^{2}$, we have

$$
\begin{aligned}
t_{1} y\left(t_{1}\right)= & \left(1+\xi \pi+w \pi^{2}\right)\left(1+\xi y(\pi)+y\left(w \pi^{2}\right)\right) \\
\equiv & 1+\xi(\pi+y(\pi))+\left(w \pi^{2}+y\left(w \pi^{2}\right)+\xi^{2} \pi y(\pi)+\xi \pi y\left(w \pi^{2}\right)\right. \\
& +\xi w \pi^{2} y(\pi) \quad\left(\bmod \mathcal{P}_{L_{0}}^{4}\right) \\
\equiv & 1+\xi\left(\pi^{2}+\pi^{3}\right)+\xi^{2}\left(\pi^{2}+\pi^{3}\right) \quad\left(\bmod \mathcal{P}_{L_{0}}^{4}\right) \\
\equiv & 1+\pi^{2}+\pi^{3}\left(\bmod \mathcal{P}_{L_{0}}^{4}\right) .
\end{aligned}
$$

The above results together with the fact that $\pi y(\pi)=\pi^{2}\left(1+\pi+\pi^{2}+\pi^{3}+\cdots\right)$ imply that

$$
k\left(c_{1}\right)=\frac{t_{2} x\left(t_{2}\right)}{t_{1} \pi y\left(t_{1} \pi\right)}=\frac{1}{\pi^{2}}\left(1+\pi+\pi^{2}+u \pi^{4}\right),
$$

for some $u \in \mathcal{O}_{L}^{*}$ (here we have $\mathcal{O}_{L}^{*}$ instead of $\mathcal{O}_{L_{0}}^{*}$ because $k\left(c_{1}\right) \in L^{*}$ ). This completes the proof of statement (1).

To prove statement (2), we use the identical method and obtain, modulo $\mathcal{P}_{L_{0}}^{6}$,

$$
\begin{aligned}
t_{2} x y\left(t_{2}\right) & \equiv 1+\xi \pi^{4} \\
\frac{x(\pi)}{\pi} & \equiv 1+\pi^{3}+\pi^{4}+\pi^{5}
\end{aligned}
$$


and

$$
\frac{x\left(t_{1}\right)}{t_{1}} \equiv 1+\xi \pi^{4}+\pi^{5}
$$

Therefore, modulo $\mathcal{P}_{L}^{6}$ (here $\left.k\left(c_{2}\right) \in L^{*}\right)$,

$$
\begin{aligned}
k\left(c_{2}\right) & \equiv\left(1+\xi \pi^{4}\right)\left(1+\pi^{3}+\pi^{4}+\pi^{5}\right)\left(1+\xi \pi^{4}+\pi^{5}\right) \\
& \equiv 1+\pi^{3}+\pi^{4}
\end{aligned}
$$

as desired.

We are now in a position to compute $M_{-}$.

Lemma 3.2.3 As an abelian group, $M_{-} \cong \mathrm{Z} / 2 \oplus \mathrm{Z} / 2$.

Proof It is easy to see that in

$$
M_{-} \cong \frac{L^{*} / E^{*}}{\left\langle k\left(c_{1}\right), k\left(c_{2}\right)\right\rangle}
$$

$1=\pi_{E} \sim \pi^{2} u_{7}$ for some $u_{7} \in U_{L}^{7} \backslash U_{L^{*}}^{8}$ It now follows from Proposition 3.2.2 that in $M_{-}, 1=k\left(c_{1}\right) \sim 1+\pi+\pi^{2}+u \pi^{4}$ and $1=k\left(c_{2}\right) \sim 1+\pi^{3}+\pi^{4}+v \pi^{6}$ for some $u$, $v \in \mathcal{O}_{L}^{*}$. These elements generate $U_{L}^{1} / U_{L}^{2}$ and $U_{L}^{3} / U_{L}^{4}$. Observe that

$$
\left(1+\pi+\pi^{2}+u \pi^{4}\right)^{2} \equiv 1+\pi^{2}+\pi^{4}\left(\bmod \mathcal{P}_{L}^{8}\right)
$$

and

$$
\left(1+\pi+\pi^{2}+u \pi^{4}\right)^{4} \equiv 1+\pi^{4}+\pi^{8} \quad\left(\bmod \mathcal{P}_{L}^{10}\right)
$$


generate the filtration levels $U_{L}^{2} / U_{L}^{3}$ and $U_{L}^{4} / U_{L}^{5}$. The above calculation also gives

$$
k\left(c_{1}\right)^{4} k\left(c_{2}\right)=1+\pi^{3}+w \pi^{6}
$$

for some $w \in \mathcal{O}_{L}^{*}$. Now under the squaring map and the action of $1+x$,

$$
\left(1+\pi^{3}+w \pi^{6}\right)^{2} \equiv 1+\pi^{6} \quad\left(\bmod \mathcal{P}_{L}^{11}\right)
$$

and

$$
(1+x)\left(1+\pi^{3}+w \pi^{6}\right) \equiv 1+\pi^{7}+\pi^{8}\left(\bmod \mathcal{P}_{L}^{9}\right)
$$

generate the filtration levels $U_{L}^{6} / U_{L}^{7}$ and $U_{L}^{7} / U_{L}^{8}$, respectively. Repeatirig this process using the squaring map and the actions of $1+x, 1+y$ and $x+y$ on $k\left(c_{1}\right), k\left(c_{2}\right)$, $\ldots$, we can generate every filtration level $U_{L}^{n} / U_{L}^{n+1}$ for $n \in \mathrm{N}, n \neq 5$. It now follows that $M_{-}$is generated by $\pi$ and $1+\pi^{5}$. Since $\left|M_{-}\right| \geq 4, \pi^{2}=\pi_{E} / u_{7} \sim 1$ and $\left(1+\pi^{5}\right)^{2}=1+\pi^{10}+\cdots \in\left\langle k\left(c_{1}\right), k\left(c_{2}\right)\right\rangle$, we have, as an abelian group,

$$
M_{-}=\left\langle\pi, 1+\pi^{5} \mid \pi^{2}=\left(1+\pi^{5}\right)^{2}=1\right\rangle \cong \mathrm{Z} / 2 \oplus \mathrm{Z} / 2,
$$

as desired.

The following proposition will give a bound on the order of $M$.

Proposition 3.2.4 $|M| \geq 2^{8}$. 
Proof Let $g \in Q_{8}$ have order 4. Define

$$
M^{g}=\{z \in M \mid g(z)=z\}
$$

It follows from Snaith [32], Proposition 6.2.7 that either

(1) $M^{x} \cong \mathrm{Z} / 2 \oplus \mathrm{Z} / 2, M^{y} \cong \mathrm{Z} / 2 \oplus \mathrm{Z} / 2$, and $M^{ \pm y} \cong \mathrm{Z} / 2 \oplus \mathrm{Z} / 2$

or

(2) two of the $M^{g^{\prime}}$ s are cyclic of order greater than or equal 8 , while the third is the sum of the other two.

Case (1) cannot occur, since this would imply $\left|\operatorname{Tor}\left(\mathbf{Z} / 2 ; M_{+}\right)\right| \geq 8$, while we in fact have from the definition of $M_{ \pm}$and Proposition 3.2.3 that

$$
\operatorname{Tor}\left(\mathrm{Z} / 2, M_{+}\right)=\operatorname{Tor}\left(\mathrm{Z} / 2, M_{-}\right) \cong \mathrm{Z} / 2 \oplus \mathrm{Z} / 2 .
$$

Hence, without loss of generality, we may assume that $M^{x} \cong \mathrm{Z} / 2^{m+1}, M^{x y} \cong \mathrm{Z} / 2^{n+1}$ for $m, n \geq 2$. It follows from the proof of Snaith [32], Lemma 6.2.4, that $\left|M_{+}\right|=$ $2 \cdot\left|M^{x}+M^{y}\right|$. Since $M^{x} \cap M^{x y}=M^{Q_{0}} \cong Z / 2$,

$$
M^{x}+M^{x y} \cong \frac{\mathbf{Z} / 2^{m+1} \oplus \mathbf{Z} / 2^{n+1}}{\left\langle\left(\left(2^{m}, 2^{n}\right)\right\rangle\right.}
$$

Thus, $\left|M_{+}\right|=2 \cdot \frac{2^{m+1} \cdot 2^{n+1}}{2}=2^{m+n+2} \geq 2^{6}$. Therefore,

$$
|M|=\left|M_{+}\right| \cdot\left|M_{-}\right| \geq 2^{8},
$$


as desired.

The core of this section lies in the following proposition.

Proposition 3.2.5 As an abelian group, $M \cong \mathrm{Z} / 16 \oplus \mathrm{Z} / 16$.

Proof Recall that

$$
M=\frac{L^{*}}{\overline{k(K \operatorname{Ker}(d))}}=\frac{L^{*}}{\left\langle k\left(c_{1}\right), k\left(c_{2}\right)\right\rangle}
$$

From Proposition 3.2.2, we have

$$
k\left(c_{2}\right) \equiv 1+\pi^{3}+\pi^{4}\left(\bmod \mathcal{P}_{L}^{6}\right)
$$

as a generator of the filtration level $U_{L}^{3} / U_{L}^{4}$. Using the identities

$$
\begin{gathered}
x(\pi)=\pi\left(1+\pi^{3}+\pi^{4}+\pi^{5}+\pi^{6}+\cdots\right), \\
y(\pi)=\pi\left(1+\pi+\pi^{2}+\pi^{3}+\pi^{4}+\pi^{7}+\cdots\right)
\end{gathered}
$$

of Section 2.2 and the squaring map, we have

$$
\begin{aligned}
(1+y)\left(k\left(c_{2}\right)\right) & \equiv 1+\pi^{4}+\pi^{6} \quad\left(\bmod \mathcal{P}_{L}^{7}\right) \\
(1+y)\left(k\left(c_{2}\right)^{2}\right) & \equiv 1+\pi^{8}\left(\bmod \mathcal{P}_{L}^{12}\right) \\
k\left(c_{2}\right)^{2} & \equiv 1+\pi^{6}+\pi^{8}+\pi^{11}\left(\bmod \mathcal{P}_{L}^{12}\right)
\end{aligned}
$$

and

$$
\equiv \quad(1+x)\left(k\left(c_{2}\right)(1+y)\left(k\left(c_{2}\right)\right)\right) \equiv 1+\pi^{6}+\pi^{7}+\pi^{8}+\pi^{9} \quad\left(\bmod \mathcal{P}_{L}^{10}\right)
$$


It follows from the above that the filtration levels $U_{L}^{4} / U_{L}^{5}, U_{L}^{6} / U_{L}^{7}, U_{L}^{7} / U_{L}^{8}, U_{L}^{8} / U_{L}^{9}$, and an element of the form $1+\pi^{7}+\pi^{9}+v \pi^{10}$ for some $v \in \mathcal{O}_{L}$ can be generated. We observe that

$$
(1+x)\left(1+\pi^{7}+\pi^{9}+v \pi^{10}\right) \equiv 1+\pi^{10}+\pi^{11}+\cdots,
$$

generates $U_{L}^{10} / U_{L}^{11}$. Applying the squaring map to the generators of the filtration obtained above we can generate $U_{L}^{12} / U_{L}^{13}, U_{L}^{14} / U_{L}^{15}$, and $U_{L}^{18} / U_{L}^{19}$. To find a generator for $U_{L}^{13} / U_{L}^{14}$, we use Corollary 2.3.6, i.e.,

$$
k\left(\left(1-x^{2}\right)\left(c_{1}-c_{2}\right)\right)=1+\pi^{8}+\pi^{13}+\pi^{16}+\cdots
$$

and the fact that

$$
\left(1+\pi^{4}+\pi^{6}+w \pi^{7}\right)^{2} \equiv 1+\pi^{8}\left(\bmod \mathcal{P}_{L}^{14}\right)
$$

for some $w \in \mathcal{O}_{L}$. Now, for some $t \in \mathcal{O}_{L}$,

$$
(1+x)\left(1+\pi^{13}+t \pi^{15}\right) \equiv 1+\pi^{16}+\pi^{17} \quad\left(\bmod \mathcal{P}_{L}^{18}\right)
$$

gives a generator for the filtration level $U_{L}^{16} / U_{L}^{17}$. We recall from Corollary 2.3.3 that if $K=Q_{2}(\sqrt{3})$ and $\pi_{K}=1+\sqrt{3}$, then

$$
A=\left(1+x+x^{2}+x^{3}\right)\left(t_{2}\right) \equiv 1+\pi_{K}^{3}+\pi_{K}^{4}+\pi_{K}^{5}+\pi_{K}^{7}\left(\bmod \mathcal{P}_{K}^{8}\right)
$$


is the first few terms of an element belonging to $k(\operatorname{Ker}(d))$. Since $\pi_{K}=\pi^{4}\left(1+\pi^{4}+\right.$ $\left.\pi^{8}+\cdots\right)$, we have,

$$
A \equiv 1+\pi^{12} \quad\left(\bmod \mathcal{P}_{L}^{16}\right)
$$

The fact that for some $s \in \mathcal{O}_{L}$ there is an element of the form $1+\pi^{6}+\pi^{7}+s \pi^{9} E$ $\left\langle k\left(c_{1}\right), k\left(c_{2}\right)\right\rangle$ implies that

$$
\left(1+\pi^{6}+\pi^{7}+s \pi^{9}\right)^{2} \equiv 1+\pi^{12}+\pi^{15}\left(\bmod \mathcal{P}_{L}^{16}\right)
$$

can be generated. Hence the filtration level $U_{L}^{15} / U_{L}^{16}$ can be generated using the element of the form

$$
\left(1+\pi^{12}\right)\left(1+\pi^{12}+\pi^{15}\right) \equiv 1+\pi^{15}\left(\bmod \mathcal{P}_{L}^{16}\right)
$$

A generator for $U_{L}^{17} / U_{L}^{18}$ can be obtained using the following observations, modulo $\mathcal{P}_{L}^{18}$ :

$$
(1+y)\left(1+\pi^{13}+0 \cdot \pi^{15}+\cdots\right) \equiv 1+\pi^{16}
$$

and

$$
\left(1+\pi^{16}\right)\left(1+\pi^{16}+\pi^{17}\right) \equiv 1+\pi^{17} .
$$

In fact, using $(1+x)\left(1+\pi^{13}+0 \cdot \pi^{15}+\cdots\right)$ and

$$
\left(1+\pi^{10}+\pi^{11}+\cdots\right)^{2}=1+\pi^{18}+\pi^{19}+\cdots
$$


we can obtain $1+\pi^{17}\left(\bmod \mathcal{P}_{L}^{20}\right)$.

Now using the squaring map, all filtration levels $U_{L}^{n} / U_{L}^{n+1}$ can be generated except for the case when $n=1,2,5,9$ or $n=8 k+3$ for some $k \in N$. Using the notation $\rho=(1-x)\left(1+x y+x^{2}+(x y)^{3}\right)$ of Chapter 2 and Corollary 2.3.7, we have for $W=\mathrm{Q}_{2}(\sqrt{30})$, modulo $\mathcal{P}_{L}^{36}$,

$$
B=\rho\left(t_{2} /\left(\pi t_{1}\right)\right)^{2} \equiv 1+\pi^{28}+\pi^{30}+\pi^{32}+\pi^{33}+\pi^{35}
$$

Since we can generate $1+\pi^{6}+\pi^{11}+\cdots$ and $1+\pi^{10}+\pi^{11}+\cdots$, there is an element of $\left\langle k\left(c_{1}\right), k\left(c_{2}\right)\right\rangle$ of the form

$$
\left(1+\pi^{6}+\pi^{11}+\cdots\right)\left(1+\pi^{10}+\pi^{11}+\cdots\right)=1+\pi^{6}+\pi^{10}+0 \cdot \pi^{11}+\cdots
$$

It follows that, modulo $\mathcal{P}_{L}^{36}$, the following element can be generated

$$
\left(1+\pi^{6}+\pi^{10}+0 \cdot \pi^{11}+\cdots\right)^{8} \equiv 1+\pi^{28}+\pi^{30}+\pi^{32} .
$$

Hence there is a trivial element of $M$ whose first few terms are

$$
B\left(1+\pi^{28}+\pi^{30}+\pi^{32}\right)=1+\pi^{33}+\pi^{35}+\cdots
$$

Since we can generate $\left(1+\pi^{17}\right)^{4} \equiv 1+\pi^{33}\left(\bmod \mathcal{P}_{L}^{36}\right)$, we have

$$
\left(1+\pi^{33}\right)\left(1+\pi^{33}+\pi^{35}\right)=1+\pi^{35}+\cdots
$$


as a generator for $U_{L}^{35} / U_{L}^{36}$.

At this moment, all of the filtration levels $U_{L}^{n} / U_{L}^{n+1}$ can be generated except for the case when $n=1,2,5,9,11,19$ or 27 . This means $|M| \leq 2^{8}$. Together with the result of the previous proposition, we have $|M|=2^{8}$. The existence of an element of the form $1+\pi^{4}+0 \cdot \pi^{5}+\cdots$ in $k(\operatorname{Ker}(d))$ can be used to improve the result of Proposition 3.2.2, part (1), i.e.

$$
1=k\left(c_{1}\right)=\frac{1+\pi+\pi^{2}+0 \cdot \pi^{3}+0 \cdot \pi^{4}+\cdots}{\pi^{2}} \in M .
$$

This means that there is an $u \in \mathcal{O}_{L}$ such that, in $M$,

$$
\pi^{2}=1+\pi+\pi^{2}+u \pi^{5} .
$$

Observe that using the squaring map successively, we have, in $M$,

$$
\pi^{4}=1+\pi^{2}+\pi^{4}+\pi^{9}+\cdots
$$

and

$$
\pi^{8}=1+\pi^{4}+\pi^{8}+\pi^{10}+\cdots .
$$

It follows that in $M, 1+\pi, 1+\pi^{2}$ and $1+\pi^{9}$ can be generated by $\pi$ and the units in lower filtration levels.

Consider the following elements of $M$ :

$$
\left(1+\pi^{5}\right)^{2}=1+\pi^{10}+\pi^{13}+\cdots
$$




$$
\begin{aligned}
& \left(1+\pi^{5}\right)^{4}=1+\pi^{18}+\pi^{20}+\cdots \\
& \left(1+\pi^{5}\right)^{8}=1+\pi^{26}+\pi^{28}+\cdots
\end{aligned}
$$

and

$$
\left(1+\pi^{5}\right)^{16}=1+\pi^{34}+\cdots \sim 1
$$

The above facts imply that for $n \geq 11$, all the elements of the form $I+\pi^{n}$ in $M$ can be generated by $\pi$ and $1+\pi^{5}$. As $\pi$ and $1+\pi^{5}$ generate $M$, and $\left(1+\pi^{5}\right)^{16} \sim 1 \in M$, we must have $\pi^{16} \sim 1 \in M$.

Therefore, as an abelian group,

$$
M=\left\langle\pi, 1+\pi^{5} \mid \pi^{16}=\left(1+\pi^{5}\right)^{16}=1\right\rangle \cong \mathrm{Z} / 16 \oplus \mathrm{Z} / 16
$$

We should also note that in this case

$$
\left.M_{+}=\left\langle\pi^{2},\left(1+\pi^{5}\right)^{2}\right)\right\rangle \cong \mathbf{Z} / 8 \oplus \mathbf{Z} / 8
$$

and

$$
M_{-}=\left\langle\pi^{8},\left(1+\pi^{5}\right)^{4}\right\rangle \cong \mathrm{Z} / 2 \oplus \mathrm{Z} / 2
$$

as in Lemma 3.2.3. The action of $Q_{8}$ on $M$ is given by $x(1,0)=(9,5), x(0,1)=(8,7)$, $y(1,0)=(3,0)$ and $y(0,1)=(0,3)$.

Corollary 3.2.6 $[M]$ is trivial in $\mathcal{C L}\left(\mathrm{Z}\left[Q_{8}\right]\right) \cong \mathrm{Z} / 2$. 
Proof Consider the induction map

$$
\operatorname{Ind}_{(y)}^{Q_{8}}: \mathcal{C L}\left(\mathrm{Z}\left[Q_{8}\right]\right) \rightarrow \mathcal{C L}\left(\mathrm{Z}\left[Q_{8}\right]\right)
$$

which sends a module $P$ io $\mathrm{Z}\left[Q_{8}\right] \otimes_{\mathrm{z}(y)]} P$. Since $\langle y\rangle \cong \mathrm{Z} / 4, \mathcal{C L}(\mathrm{Z}[\langle y\rangle])=0$, $\mathrm{Z} / 16=\langle(1,0)\rangle$ is a $\mathrm{Z}[\langle y\rangle]$-module, and

$$
x(\langle(1,0)\rangle) \oplus\langle(1,0)\rangle=\langle(9,5)\rangle \oplus\langle(1,0)\rangle \cong M
$$

we have $\operatorname{Ind} d_{\{y)}^{Q s}([\mathrm{Z} / 16])=[\mathrm{Z} / 16 \oplus \mathrm{Z} / 16]$, and hence

$$
[M]=[\mathrm{Z} / 16 \oplus \mathrm{Z} / 16]=0 \in \mathcal{C L}\left(\mathrm{Z}\left[Q_{8}\right]\right)
$$

as desired.

\subsection{Computation of $[X]$}

In this" section, we compute the class $[X] \in \mathcal{C L}\left(\mathrm{Z}\left[Q_{8}\right]\right)$ in term of the root number class $W_{N / Q}$ using Fröhlich's Hom-description together with results of A. Fröhlich and M. J. Taylor.

For the Hom-description we take any Galois extension of number ficlds, $E / Q$, such that $N(\sqrt{-1}) \subset E$. Then 


$$
\mathcal{C L}\left(\mathrm{Z}\left[Q_{8}\right]\right) \cong \frac{\operatorname{Hom}_{\Omega_{Q}}\left(R\left(Q_{8}\right), J^{*}(E)\right)}{\operatorname{Hom}_{\Omega_{Q}}\left(R\left(Q_{8}\right), E^{*}\right) \operatorname{Det}\left(U\left(\mathrm{Z}\left[Q_{8}\right]\right)\right)}
$$

Since $\Omega_{\mathrm{Q}}$ acts trivially on $R\left(Q_{\mathrm{B}}\right)$, all characters are integer-valued. We have

$$
H o m_{\Omega_{Q}}\left(R\left(Q_{8}\right), E^{*}\right) \cong H o m\left(R\left(Q_{8}\right), Q^{*}\right)
$$

and

$$
H o m_{\Omega_{\mathrm{q}}}\left(R\left(Q_{8}\right), J^{*}(E)\right) \cong H o m\left(R\left(Q_{8}\right), J^{*}(E)^{\Omega_{\mathfrak{Q}}}\right)
$$

Also, the p-part of the adèles is $E \otimes_{\mathrm{Q}} \mathbf{Q}_{\mathrm{p}} \cong \prod_{\mathcal{P} \mid p} E_{\mathcal{P}}$ and $\Omega_{\mathrm{Q}}$ acts trivially on the factors with stabilizer $\Omega_{Q_{p}}$, acting on each $E_{\mathcal{P}}$ component-wise $\left(\left(E \otimes_{Q} Q_{p}\right)^{\Omega_{Q}} \cong\right.$ $E_{\mathcal{P}}^{\Omega_{Q_{p}}}=Q_{p}$ for any choice of $\left.\mathcal{P} \mid p\right)$. Hence

$$
\operatorname{Hom}_{\Omega_{\mathbf{Q}}}\left(R\left(Q_{8}\right), J^{*}(E)\right) \cong \prod_{p}{ }^{\prime} H o m\left(R\left(Q_{8}\right), Q_{p}^{*}\right)
$$

The above weak product includes one $p=\infty$ factor.

Now $X \otimes \mathrm{Q} \cong N \cong \mathrm{Q}\left[Q_{8}\right]<a>$ for $a \in O_{N} \backslash\{0\}$ and $X \otimes_{\mathrm{z}} \mathrm{Z}_{2} \cong \mathrm{Z}_{2}\left[Q_{8}\right]<a_{2}>$ with $a_{2}=2^{t}(1+\sqrt{3}+\sqrt{10}+\sqrt{30}+\alpha) \in 8 O_{L}$. The fact that $\left.Z_{2}\left[Q_{8}\right]<a_{2}\right\rangle$ is a free $\mathbf{Z}_{2}\left[Q_{8}\right]$-module is proved in the following lemma.

Lemma 3.3.1 The module $\mathrm{Z}_{2}\left[Q_{8}\right](1+\sqrt{3}+\sqrt{10}+\sqrt{30}+\alpha)$, a $\mathbf{Z}_{2}\left[Q_{8}\right]$-submodule of $\mathcal{O}_{L}$ generated by $1+\sqrt{3}+\sqrt{10}+\sqrt{30}+\alpha$, is free over $\mathrm{Z}_{2}\left[Q_{8}\right]$. 
Proof Let $\chi_{1}, \chi_{2}: Q_{8} \longrightarrow \pm 1$ be homomorphisms defined by

$$
\begin{aligned}
& \chi_{1}(x)=-1, \quad \chi_{1}(y)=1 \\
& \chi_{2}(y)=-1, \quad \chi_{2}(x)=1 .
\end{aligned}
$$

The irreducible complex representations of $Q_{8}$ are given by $1, \chi_{1}, \chi_{2}, \chi_{1} \chi_{2}$ and a unique irreducible two-dimensional representation $\nu$. For each irreducible representation $\chi$ of $Q_{8}$, we let $e_{\chi}=\frac{\operatorname{dim}(\chi)}{8} \sum_{s \in Q_{8}} \chi\left(g^{-1}\right) g$ be its associated idempotent. Then, as in $\operatorname{Kim}[22]$, Lemma 2.4, $\gamma=\sum_{x} \gamma_{x}$ generates a free module over $\mathrm{Z}_{2}\left[Q_{8}\right]$ where $\chi$ runs through all irreducible representations of $Q_{B}$ and $\gamma_{X}$ is a generator for $e_{X} \mathcal{O}_{L}$ over $e_{x} \mathrm{Z}_{2}\left[Q_{8}\right]$.

We consider

$$
e_{1} \mathcal{O}_{L}=\frac{1}{8} \operatorname{Tr}_{L / Q_{2}}\left(\mathcal{O}_{L}\right)=\mathrm{Z}_{2} \cdot 1
$$

and

$$
\begin{aligned}
e_{x_{1}} \mathcal{O}_{L} & =\frac{1}{8}\left(\sum_{g \in Q_{t}} \chi_{1}\left(g^{-1}\right) g\right) \mathcal{O}_{L} \\
& =\frac{1}{8}(1-x) T r_{L / Q_{2}(\sqrt{10})}\left(\mathcal{O}_{L}\right) \\
& =\mathbf{Z}_{2} \cdot \sqrt{10}
\end{aligned}
$$

Similarly, we find $\gamma_{x_{2}}=\sqrt{3}, \gamma_{x_{1} x_{2}}=\sqrt{30}$ and $\gamma_{\nu}=\alpha$. Thus, $\gamma=1+\sqrt{3}+\sqrt{10}+$ $\sqrt{30}+\alpha$, as desired. 
At odd primes $p$

$$
X_{p}=X \otimes \mathbf{z} \mathbf{Z}_{p}=\mathbf{Z}_{p}\left[Q_{8}\right]<a_{p}>\cong \prod_{\mathcal{P} \mid p} O_{N_{p}}
$$

where $a_{p} \in O_{N_{p}}$ for some $\mathcal{P}$ over $p$ and $O_{N_{p}}=Z_{p}\left[G\left(N_{\mathcal{P}} / Q_{p}\right)\right]<a_{p}>$. Hence we have homomorphisms $R\left(Q_{8}\right) \rightarrow \prod_{\mathcal{P} \mid \mathrm{p}} N_{\mathcal{P}}^{*}$

$$
x \longmapsto \frac{\left(a_{p} \mid x\right)}{(a \mid x)}
$$

and $R\left(Q_{8}\right) \rightarrow N_{2}^{*}$

$$
\chi \longmapsto \frac{\left(a_{2} \mid \chi\right)}{(a \mid x)}
$$

Here $(\cdot \mid \cdot)$ denotes the resolvent homomorphism which is defined in the following manner. If $\Lambda$ is any ring with a $G(N / Q)$-action and $u \in \Lambda$, then for $\chi \in R\left(Q_{8}\right)$,

$$
(u \mid \chi)=\operatorname{det}\left(\sum_{g \in G(N / Q)} g(u) \chi\left(g^{-1}\right)\right)
$$

If $\omega \in \Omega_{Q}$, then

$$
\begin{aligned}
\omega(u \mid \chi) & =\operatorname{det}\left(\sum_{g \in G(N / Q)} \omega g(u) \omega\left(\chi\left(g^{-1}\right)\right)\right) \\
& =\operatorname{det}\left(\sum_{\omega g} \omega g(u) \chi\left((\omega g)^{-1}\right) \chi(\omega g) \omega\left(\chi\left(g^{-1}\right)\right)\right) .
\end{aligned}
$$

If $\chi$ is irreducible and 1-dimensional, then $\omega\left(\chi\left(g^{-1}\right)\right)=\chi\left(g^{-1}\right)$ and $\chi(\omega g)=\chi(\omega) \chi(g)$, so $w(u \mid \chi)=(u \mid \chi) \operatorname{det}(\omega(\chi(u)))$ in this case. This formula is true in general (see 
Fröhlich [9], page 30). This means that when $\chi=\nu$, each resolvent is an $\Omega_{\mathrm{Q}}$-map

$$
\omega(a \mid \nu)=(a \mid \nu)
$$

as $\operatorname{det}(\nu)=1$. But for the one-dimensional characters $\chi$ one must be careful to maintain quotients like $\frac{\left(a_{p} \mid x\right)}{(a \mid x)}$, so that the $\operatorname{det}(\chi(\omega))$ 's cancel. Hence the Hom-description of $[X]$ is

$$
x \longmapsto \begin{cases}\frac{\left(a_{p} \mid x\right)}{(a \mid x)} & \text { if } p \text { is finite } \\ 1 & \text { if } p=\infty .\end{cases}
$$

If $p$ is finite, then $\tau_{N / Q}(\chi)=\prod_{p} \tau_{p}\left(\chi_{p}\right)$, and according to Fröhlich [9], pagc 119, Theorem 20B(ii), for $\omega \in \Omega_{Q}$,

$$
\omega\left(\tau_{N / Q}(\chi)\right)=\tau_{N / Q}(\chi) \operatorname{det}(\chi)(\omega)
$$

In addition, the results of Fröhlich [11], show that $\frac{\left(a_{p} \mid x\right)}{\left(\tau_{p}\left(x_{p}\right)\right)}$ is unit-valued in $\prod_{\mathcal{P} \mid p} O_{N_{p}}^{*}$ for $p$ odd (in this case, $N_{p} / Q_{p}$ is tame). But $\left|r_{q}\left(\chi_{q}\right)\right|$ is a power of $q$, so this is unit-valued, and so for $p \neq 2$,

$$
\begin{aligned}
\frac{\left(a_{p} \mid \chi\right)}{\tau_{p}(\chi)} & \in H o m_{\Omega_{Q}}\left(R\left(Q_{8}\right), \prod_{\mathcal{P} \mid p} O_{E_{p}}^{*}\right) \\
& =\operatorname{Det}\left(Z_{p}\left[Q_{8}\right]^{*}\right)
\end{aligned}
$$

Therefore, at each finite $p \neq 2$ we may divide by $\chi \longmapsto \frac{\left(a_{p} \mid x\right)}{T(x)}$ to obtain a Hom- 
representation of the form

$$
x \longmapsto \begin{cases}\frac{\left(a_{2} \mid x\right)}{(a \mid x)} & \text { at } p=2 \\ \frac{\tau(x)}{(a \mid x)} & \text { at } p \neq 2, \infty \\ 1 & \text { at } p=\infty\end{cases}
$$

We observe that the function

$$
\chi \mapsto \frac{\tau(\chi)}{(a \mid \chi)}
$$

lies in $H o m_{\Omega_{Q}}\left(R\left(Q_{8}\right), E^{*}\right)$ so we can divide by it, to change the Hom-representation of $[X]$ to

$$
\chi \longmapsto \begin{cases}\frac{\left(a_{2} \mid x\right)}{\tau(x)} & \text { at } p=2 \\ 1 & \text { at } p \neq 2, \infty \\ \frac{(a \mid x)}{\tau(x)} & \text { at } p=\infty\end{cases}
$$

Now according to Fröhlich [12], at $\infty$ the sign of $(a \mid \chi)$ is equal to the sign of $W_{\infty}(\nu)$ and the absolute norm of the conductor of a symplectic representation is a square. So the sign of $\tau(\nu) W_{\infty}(\nu)$ is the same as that of $W_{\text {finite }} W_{\infty}(\nu)=W_{\mathrm{Q}}(\nu)$, the Artin conductor. Therefore, since $W_{Q}(\chi)=1$ if $\operatorname{dim}(\chi)=1$,

$$
\chi \longmapsto \begin{cases}1 & \text { at } p \neq \infty \\ \frac{(a \mid x)}{\tau(x)} W_{Q}(x) & \text { at } p=\infty\end{cases}
$$

is in $H o m_{\Omega_{0}}\left(R\left(Q_{8}\right), E^{*}\right)$ and its sign at $\infty$ on $\chi=\nu$ is

$$
\operatorname{sign}\left(\frac{(a \mid \chi)}{\tau(\chi)} W_{\mathrm{Q}}(\chi)\right)=\operatorname{sign}\left(\frac{W_{\mathrm{Q}}(\chi)}{W_{\infty}(\chi) \tau(\chi)}\right)=1
$$


(see Snaith [33], page 336, Chinburg [3], page 19, and Taylor [38], page 9).

Therefore, as $W_{Q}(x)=1$ if $\operatorname{dim}(\chi)=1$,

$$
x \longmapsto \begin{cases}\frac{\left(\left(_{2} \mid x\right)\right.}{\tau(x)} & \text { at } p=2 \\ 1 & \text { otherwise }\end{cases}
$$

is a Hom-representative for $[X]-W_{N / Q}$.

Now if we define

$$
h: R\left(Q_{8}\right) \longrightarrow \mathrm{Z}_{2}^{*} \text { by } h(\chi) \cdot 2^{\beta(x)}=\frac{\left(a_{2} \mid \chi\right)}{\tau(\chi)}
$$

and

$$
\chi_{+}:(\mathrm{Z} / 8)^{*} \longrightarrow\{ \pm 1\} \text { by } \chi_{+}(-1)=1 \text { and } \chi_{+}(3)=-1 \text {, }
$$

then the class of $[h]$ is

$$
A=[X]-W_{N / Q}=h(\nu) \cdot \chi_{+}\left(h\left(1+\chi_{1}+\chi_{2}+\chi_{1} \chi_{2}\right)\right) \in\{ \pm 1\}
$$

Note that in our computation, we discard the power of 2 . We now have

$$
A \equiv \frac{\left(a_{2} \mid \chi\right)}{\tau(\chi)} \chi_{+}\left(\frac{\left(a_{2} \mid 1+\chi+\chi_{1}+\chi_{2}+\chi_{1} \chi_{2}\right)}{\tau\left(1+\chi_{1}+\chi_{2}+\chi_{1} \chi_{2}\right)}\right) \quad(\bmod 4)
$$

Before going further in this direction, we use the same method of Snaith [32], Lemma 6.3.3, to prove the following useful lemma. 
Lemma 3.3.2 Let $a_{2}=2^{t}(1+\sqrt{3}+\sqrt{10}+\sqrt{30}+\alpha)$ be as in Lemma 3.9.1. Then the resolvent of $a_{2}$ is given by the following formulae:

$$
\begin{aligned}
& \left(a_{2} \mid 1\right)=2^{t+3} \\
& \left(a_{2} \mid \chi_{1}\right)=2^{t+3} \sqrt{10} \\
& \left(a_{2} \mid \chi_{2}\right)=2^{t+3} \sqrt{3} \\
& \left(a_{2} \mid \chi_{1} \chi_{2}\right)=2^{t+3} \sqrt{30} \\
& \left(a_{2} \mid \nu\right)=2^{2 t+2} \alpha^{2}\left(1+u_{1}^{2}\right)\left(1+u_{2}^{2}\right)
\end{aligned}
$$

where $x(\alpha)=u_{1} \alpha$ and $y(\alpha)=u_{2} \alpha$.

Proof For convenience, let us suppose that $t=0$. From Lemma 2.1.2, we know that $u_{1}=(1-\sqrt{10}) / 3$ and $u_{2}=(\sqrt{3}-1) /(\sqrt{30} v)$ where $v^{2}=-1 / 15$. We now have the following formulae:

$$
\begin{aligned}
& a_{2}=1+\sqrt{3}+\sqrt{10}+\sqrt{30}+\alpha, \\
& x\left(a_{2}\right)=1+\sqrt{3}-\sqrt{10}-\sqrt{30}+u_{1} \alpha, \\
& y\left(a_{2}\right)=1-\sqrt{3}+\sqrt{10}-\sqrt{30}+u_{2} \alpha, \\
& x y\left(a_{2}\right)=1-\sqrt{3}-\sqrt{10}+\sqrt{30}+u_{1} x\left(u_{2}\right) \alpha, \\
& x^{2}\left(a_{2}\right)=1+\sqrt{3}+\sqrt{10}+\sqrt{30}-\alpha,
\end{aligned}
$$




$$
\begin{aligned}
& x^{3}\left(a_{2}\right)=1+\sqrt{3}-\sqrt{10}-\sqrt{30}-u_{1} \alpha \\
& x^{2} y\left(a_{2}\right)=1-\sqrt{3}+\sqrt{10}-\sqrt{30}-u_{2} \alpha \\
& x^{3} y\left(a_{2}\right)=1-\sqrt{3}-\sqrt{10}+\sqrt{30}-u_{1} x\left(u_{2}\right) \alpha .
\end{aligned}
$$

Therefore

$$
\begin{aligned}
\left(a_{2} \mid 1\right) & =\sum_{g \in Q_{s}} g\left(a_{2}\right) \\
& =8 \\
\left(a_{2} \mid \chi_{1}\right) & =\sum_{x_{1}(s)=1} g\left(a_{2}\right)-\sum_{x_{1}(g)=-1} g\left(a_{2}\right) \\
& =8 \sqrt{10}, \\
\left(a_{2} \mid x_{2}\right) & =\sum_{x_{2}(g)=1} g\left(a_{2}\right)-\sum_{x_{1}(g)=-1} g\left(a_{2}\right) \\
& =8 \sqrt{3},
\end{aligned}
$$

and

$$
\begin{aligned}
\left(a_{2} \mid \chi_{1} \chi_{2}\right) & =\sum_{\chi_{1}(g) \times_{2}(g)=1} g\left(a_{2}\right)-\sum_{\chi_{1}(g) \times \times_{2}(g)=-1} g\left(a_{2}\right) \\
& =8 \sqrt{30} .
\end{aligned}
$$

The matrices representing $\left\{\nu(g) \mid g \in Q_{8}\right\}$ are given by $\left(i^{2}=-1\right)$

$$
\nu(1)=\left(\begin{array}{cc}
1 & 0 \\
0 & 1
\end{array}\right), \nu(x)=\left(\begin{array}{cc}
i & 0 \\
0 & -i
\end{array}\right)
$$




$$
\nu(y)=\left(\begin{array}{cc}
0 & 1 \\
-1 & 0
\end{array}\right), \nu(x y)=\left(\begin{array}{cc}
0 & i \\
i & 0
\end{array}\right),
$$

and $\nu\left(x^{2} g\right)=-\nu(g)$. Hence we find that

$$
\begin{aligned}
\left(a_{2} \mid \nu\right) & =\operatorname{det}\left(\begin{array}{cc}
2 \alpha\left(1+i u_{1}\right) & 2 u_{2} \alpha\left(1+i u_{1}\right) \\
2 u_{2} \alpha\left(i u_{1}-1\right) & 2 \alpha\left(1-i u_{1}\right)
\end{array}\right) \\
& =4 \alpha^{2}\left(1+u_{1}^{2}\right)\left(1+u_{2}^{2}\right)
\end{aligned}
$$

as desired.

Now,

$$
W_{\text {finite }}(x)=\prod_{p \text { finite }} W_{Q_{p}}\left(\chi_{p}\right)=\sqrt{f(x)} \cdot \tau(x)
$$

where $f(\chi)$ is the Artin conductor associated to the character $\chi$ of $Q_{8}$. Recall that in Lemma 2.1.1, $H=\mathrm{Q}\left(\sqrt{d_{1}}, \sqrt{d_{2}}\right)$ is defined to be the bi-quadratic subfield of the quaternion extension $N / \mathrm{Q}$ with $d_{1} \equiv 3 \quad(\bmod 8)$ and $d_{2} \equiv 10 \quad(\bmod 16)$. We have

$$
f\left(1+\chi_{1}+\chi_{2}+\chi_{1} \chi_{2}\right)=f\left(\operatorname{Ind}_{G(N / H)}^{G(N / Q)}(1)\right)=D_{1 ! / Q}
$$

and

$$
\left.f\left(2 \nu+1+\chi_{1}+\chi_{2}+\chi_{1} \chi_{2}\right)=f(\operatorname{Ind} G(N) G(Q))\right)=D_{N / Q} \text {. }
$$

It follows that $f(\nu)^{2}=\frac{D_{N / 9}}{D_{H / 9}}$. According to Martinet [25], Proposition 6 (proof),

$$
D_{N / Q}=\prod_{\substack{p \text { ramified } \\ \text { in } N / Q}} p^{4} \cdot D_{H / Q} .
$$


Hence (the odd part)

$$
f(\nu)=\prod_{\substack{\neq p \text { ramified } \\ \text { in } N / Q}} p^{2} \equiv 1 \quad(\bmod 4)
$$

Using all of the above relations together with the fact that $\operatorname{Gal}(N / H)=\left\langle x^{2}\right\rangle$, we have

$$
A=\frac{\left(a_{2} \mid \nu\right) \sqrt{f(\nu)}}{\prod_{p \text { finite }} W_{Q_{p}}\left(\nu_{p}\right)} \cdot \chi+\left(\frac{\left(a_{2} \mid 1+\chi_{1}+\chi_{2}+\chi_{1} \chi_{2}\right)}{W_{\text {finite }}\left(I_{n d} d_{x^{2}>}(1)\right.} \cdot \sqrt{D_{H / Q}}\right) .
$$

We are now in a position to state and prove the main result of this section.

Theorem 3.3.3 In $\mathcal{C L}\left(\mathrm{Z}\left[Q_{8}\right]\right)$,

$$
[X]= \begin{cases}-W_{N / Q} & \text { if } \alpha^{2}=1+\sqrt{3}+\sqrt{10} / 10+\sqrt{30} / 10 \\ W_{N / Q} & \text { if } \alpha^{2}=-1-\sqrt{3}-\sqrt{10} / 10-\sqrt{30} / 10\end{cases}
$$

Proof Since

$$
[X]-W_{N / Q}=A \in\{ \pm 1\} \cong \mathcal{C L}\left(Z\left[Q_{8}\right]\right)
$$

to prove the theorem it suffices to show that

$$
A= \begin{cases}-1 & \text { if } \alpha^{2}=1+\sqrt{3}+\sqrt{10} / 10+\sqrt{30} / 10 \\ 1 & \text { if } \alpha^{2}=-1-\sqrt{3}-\sqrt{10} / 10-\sqrt{30} / 10 .\end{cases}
$$

To compute $A$, we analyse the formula given prior to the statement of the theorem.

Since $W_{\infty}\left(I n d_{\left\langle x^{2}>\right.}^{P_{d}}(1)\right)= \pm 1$ and $I n d_{\left\langle x^{2}>\right.}^{Q_{i}}(1)$ is orthogonal, we have

$$
W_{\text {finite }}\left(I n d_{\left\langle x^{2}>\right.}^{Q_{e}}(1)\right)=\frac{W_{Q}\left(\operatorname{In} d_{<x^{2}>}^{Q_{k}}(1)\right)}{W_{\infty}\left(\operatorname{In} d_{\left\langle x^{2}>\right.}^{q}(1)\right)}
$$




$$
= \pm 1
$$

Thus $\chi_{+}\left(W_{\text {finite }}\left(I n d_{<x^{2}>}^{Q_{s}}(1)\right)\right)=1$ and

$$
A=\frac{\left(a_{2} \mid \nu\right) \sqrt{f(\nu)}}{\prod_{p} \text { finite } W_{Q_{p}}\left(\nu_{p}\right)} \cdot \chi_{+}\left(\left(a_{2} \mid 1+\chi_{1}+\chi_{2}+\chi_{1} \chi_{2}\right) \cdot \sqrt{D_{H / Q}}\right) .
$$

In the next lemma we will show that $W_{Q_{2}}\left(\nu_{2}\right)=-1$. Hence

$$
W_{\text {finite }}(\nu)=-\prod_{2 \neq p_{\text {in } N / Q}} W_{Q_{p}}\left(\nu_{p}\right)
$$

It follows from Lemma 3.3.2 that

$$
\left(a_{2} \mid 1+\chi_{1}+\chi_{2}+\chi_{1} \chi_{2}\right)=2^{4 t+12} \cdot 15
$$

and

$$
\left(a_{2} \mid \nu\right)= \begin{cases}2^{2 t+4} \cdot 1 & \text { if } \alpha^{2}=1+\sqrt{3}+\sqrt{10} / 10+\sqrt{30} / 10 \\ 2^{2 t+4} \cdot(-1) & \text { if } \alpha^{2}=-1-\sqrt{3}-\sqrt{10} / 10-\sqrt{30} / 10\end{cases}
$$

The former implies that

$$
\chi_{+}\left(a_{2} \mid 1+\chi_{1}+\chi_{2}+\chi_{1} \chi_{2}\right)=\chi_{+}(15)=1
$$

Now if we let $D_{0}$ be the odd part of $\sqrt{D_{H / Q}}$, then it follows from Martinet [25] (see also Kim [22], Lemma 3.1) that

$$
\prod_{\substack{2 \neq p \text { ramified } \\ \text { in } N / Q}} W_{Q_{p}}\left(\nu_{p}\right) \equiv\left(\frac{2}{D_{0}}\right) \prod_{2 \neq p \text { ramified }} p(\bmod 4),
$$


Hence, in $\mathcal{C L}\left(\mathrm{Z}\left[Q_{8}\right]\right) \cong\{ \pm 1\}$,

$$
A= \begin{cases}-\left(\frac{2}{D_{0}}\right) \chi_{+}\left(D_{0}\right) & \text { if } \alpha^{2}=1+\sqrt{3}+\sqrt{10} / 10+\sqrt{30} / 10 \\ \left(\frac{2}{D_{0}}\right) \chi_{+}\left(D_{0}\right) & \text { if } \alpha^{2}=-1-\sqrt{3}-\sqrt{10} / 10-\sqrt{30} / 10\end{cases}
$$

It can be easily seen from the definitions of the Jacobi symbol and $\chi_{+}$that $\left(\frac{2}{D_{0}}\right)=$ $\chi_{+}\left(D_{0}\right)$. Therefore, in $\mathcal{C L}\left(\mathrm{Z}\left[Q_{8}\right]\right)$

$$
[A]=\left\{\begin{array}{cl}
-1 & \text { if } \alpha^{2}=1+\sqrt{3}+\sqrt{10} / 10+\sqrt{30} / 10 \\
1 & \text { if } \alpha^{2}=-1-\sqrt{3}-\sqrt{10} / 10-\sqrt{30} / 10
\end{array}\right.
$$

as desired.

Lemma 3.3.4 Let $\nu$ be the two-dimensional irreducible complex representation of $Q_{8} \cong \operatorname{Gal}(N / Q)$. Then the local Artin root number of $\nu$ satisfies

$$
W_{Q_{2}}\left(\nu_{2}\right)=-1
$$

Proof As in Chinburg, Kolster, Pappas and Snaith [6], for $V=Q_{2}(\sqrt{10})$ we let $\varphi$ be a character of $\operatorname{Gal}(L / V)$ of order 4. Identical arguments show that $W_{V}(\varphi)=1$ (in their paper, $\varphi$ is denoted by $\left.\chi_{\nu_{2}}\right)$. Since $\operatorname{Gal}(L / V) \cong \mathrm{Z} / 4$ and $\nu_{2}=\operatorname{Ind} d_{\mathrm{z} / 4}^{Q_{8}}(\varphi)$, we have $\operatorname{In} d_{z / 1}^{Q} j_{1}(\varphi-1)=\nu_{2}-\operatorname{Ind} q_{z / 4}^{Q q}(1)$. Hence

$$
W_{\mathrm{Q}_{2}}\left(\nu_{2}\right)=W_{\mathrm{Q}_{2}}\left(\operatorname{In} d_{\mathrm{Z} / 4}^{Q_{\mathrm{f}}}(1)\right) \cdot W_{\mathrm{Q}_{2}}\left(\operatorname{In} d_{\mathrm{Z} / 4}^{Q_{\mathrm{g}}}(\varphi-1)\right)
$$




$$
\begin{aligned}
& =W_{Q_{2}}\left(I n d_{Z / 4}^{\left.Q_{d}(1)\right)}\right) \cdot W_{K}(\varphi) \\
& =W_{Q_{2}}\left(I n d_{Z / 4}^{Q_{B}}(1)\right) .
\end{aligned}
$$

Since $\operatorname{In} d_{z / 4}^{Q_{B}}(1)=1+\operatorname{In} \int_{z / 2}^{Q_{s}}(\theta)$ where $\theta$ is the nontrivial character of $\operatorname{Gal}\left(V / Q_{2}\right) \cong$ $\mathrm{Z} / 2$, we have

$$
W_{Q_{2}}\left(\nu_{2}\right)=W_{Q_{2}}(\theta)
$$

It now follows from Snaith [34], page 266, that if we define $l: \Omega_{Q_{2}} \rightarrow\{ \pm 1\}$ by $g \mapsto g(\sqrt{10}) /(\sqrt{10})$, then

$$
W_{\mathbf{Q}_{2}}\left(\nu_{2}\right)=W_{\mathbf{Q}_{2}}(l(10))=-1
$$

as desired.

\subsection{Computation of $\left[\operatorname{Ker}\left(k^{\prime}\right)\right]$}

In this section we shall eveluate the class of $\operatorname{Ker}\left(k^{\prime}\right)$ in

$$
\mathcal{C L}\left(\mathrm{Z}\left[Q_{8}\right]\right) \cong D\left(\mathrm{Z}\left[Q_{8}\right]\right) \cong(\mathrm{Z} / 4)^{*}
$$

Using the results of Snaith [32], Sections 6.3.2-3, we have that a Hom-description representative for $\left[K \operatorname{Ker}\left(k^{\prime}\right)\right]$ is given by a function $g$ such that $g(T) \in \mathbf{Z}_{2}^{*}$ and

$$
2^{\complement} g(T)=\frac{\left(a_{2} \mid T\right)}{\left(\log _{2}\left(k\left(x_{0}\right)\right) \mid T\right)}
$$


where $T \in R\left(Q_{8}\right), \epsilon \in \mathrm{N}, a_{2}=1+\sqrt{3}+\sqrt{10}+\sqrt{30}+\alpha$ as defined in the previous section, and $x_{0}=2^{s}\left(\sigma\left(t_{2}\right)+\tau\left(c_{1}\right)+\lambda\left(c_{1}\right)+\rho\left(c_{2}\right)+\left(1-x^{2}\right)\left(c_{1}-c_{2}\right)\right)$ for some integer $s \leq 0$ and $c_{1}, c_{2}, i_{1}, t_{2}, \sigma, \tau, \lambda, \rho$ are defined as in Section 2.1.1-2. It follows from Taylor [38], page 88 , that the class

$$
[g] \in D\left(\mathrm{Z}\left[Q_{8}\right]\right) \cong(\mathrm{Z} / 4)^{*}
$$

is given by the formula

$$
[g] \equiv g(\nu)(-1)^{(1 / 4) \log _{2}\left(g\left(1+x_{1}+x_{2}+x_{1} x_{2}\right)\right)} \quad(\bmod 4) .
$$

In addition, $(-1)^{(1 / 4) \log _{2}\left(g\left(1+x_{1}+x_{2}+x_{1} x_{2}\right)\right)}$ depends only on $g(1), g\left(\chi_{1}\right), g\left(\chi_{2}\right)$ and $g\left(\chi_{1} \chi_{2}\right)$ modulo eight, since

$$
(-1)^{(1 / 4) \log _{2}(1+1 w)}=(-1)^{w-2 w^{2}}=(-1)^{w} .
$$

Lemma 3.4.1 If $g: R\left(Q_{8}\right) \longrightarrow \mathrm{Z}_{2}^{*}$ is the function of 9.1 then

$$
g(1) \in 1+8 \mathrm{Z}_{2} \text {. }
$$

Proof As in Snaith [32], Lemma 6.3.4 (proof), we have

$$
\left(\log _{2}\left(k\left(x_{0}\right)\right) \mid 1\right)=2^{\rho+3} \log _{2}\left(k\left(\sigma c_{2}\right)\right)
$$

It follows from Lemma 2.3.2 that $k\left(\sigma c_{2}\right)=\sigma\left(t_{2}^{2}\right)=\left(13+2^{5} \delta\right)^{2}$ for some $\delta \in \mathbf{Z}_{2}$. Hence

$$
\log _{2}\left(k\left(\sigma c_{2}\right)\right)=2 \log _{2}\left(13+2^{5} \delta\right)
$$




$$
\begin{aligned}
& =2 \log _{2}\left(1+4\left(3+2^{3} \delta\right)\right) \\
& =2\left(4\left(3+2^{3} \delta\right)-4^{2} / 2\left(3+2^{3} \delta\right)^{2}+\ldots\right)
\end{aligned}
$$

which lies in $8\left(3-2+\delta Z_{2}\right)$. Therefore, by Lemma 3.3.2, we find that

$$
\frac{\left(a_{2} \mid 1\right)}{\left(\log _{2}\left((k \otimes 1)\left(x_{0}\right)\right) \mid 1\right)} \in \frac{2^{t+3}}{2^{5+6}\left(1+S Z_{2}\right)}
$$

and $g(1) \in\left(1+\delta Z_{2}\right)^{-1}=\left(1+\delta Z_{2}\right)$, as desired.

Lemma 3.4.2 If $g: R\left(Q_{8}\right) \longrightarrow \mathrm{Z}_{2}^{*}$ is the function of 3.1 then

$$
g\left(\chi_{1}\right) \in 3+8 Z_{2}
$$

Proof As in Snaith [32], Lemma 6.3.5 (proof), we have

$$
\left(\log _{2}\left(k\left(x_{0}\right)\right) \mid \chi_{1}\right)=-2^{r+4} \log _{2}\left(k\left(\lambda b^{\prime}\right)\right)
$$

It follows from Corollary 2.3.7 that

$$
k\left(\lambda b^{\prime}\right)=\lambda\left(\pi t_{1}\right)=3+2 \sqrt{10}+2^{4} \delta
$$

for some $\delta \in Z_{2}[\sqrt{10}]$. Hence if we let $w=1+\sqrt{10}+2^{3} \delta$, then

$$
\begin{aligned}
\log _{2}\left(k\left(\lambda b^{\prime}\right)\right) & =\log _{2}(1+2 w) \\
& =2\left(w-w^{2}+4 w^{3} / 3-2 w^{4}+\cdots\right)
\end{aligned}
$$


which lies in $2(\sqrt{10})\left(3+8 \mathbf{Z}_{2}\right)$. Hence, by Lemma 3.3.2, we find that

$$
\frac{\left(a_{3} \mid \chi_{1}\right)}{\left(\log _{2}\left(k\left(x_{0}\right)\right) \mid \chi_{1}\right)} \in \frac{2^{s+3}(\sqrt{10})}{\left(-2^{s+5}\right)(\sqrt{10})\left(3+\delta Z_{2}\right)}
$$

and $g\left(\chi_{1}\right) \in\left(3+8 Z_{2}\right)^{-1}=\left(3+8 Z_{2}\right)$, as desired.

Lemma 3.4.3 If $g: R\left(Q_{8}\right) \longrightarrow Z_{2}^{-}$is the function of 3.1 then

$$
g\left(\chi_{2}\right) \in 3+8 \mathbf{Z}_{2}
$$

Proof As in Snaith [32], Lemma 6.3.6, we have

$$
\left(\log _{2}\left(k\left(x_{0}\right)\right) \mid \chi_{2}\right)=2^{s+4} \log _{2}\left(\tau c_{1}\right)
$$

It follows from Corollary 2.3.7 that

$$
\tau\left(c_{1}\right)=\tau\left(t_{2}^{2}\right)=\left(25+12 \sqrt{3}+2^{5} \delta\right)^{2}
$$

for some $\delta \in Z_{2}[\sqrt{3}]$. Hence, if we let $w=6+3 \sqrt{3}+2^{3} \delta$, then

$$
\begin{aligned}
\log _{2}\left(\tau\left(c_{1}\right)\right) & =2 \log _{2}\left(t_{2}\right) \\
& =2 \log _{2}(1+4 w) \\
& =8\left(w-2 w^{2}+\cdots\right)
\end{aligned}
$$

which lies in $(\sqrt{3})\left(3+8 Z_{2}\right)$. Therefore, by Lemma 3.3.2,

$$
\frac{\left(a_{2} \mid \chi_{2}\right)}{\left(\log _{2}\left(k\left(x_{0}\right)\right) \mid \chi_{2}\right)} \in \frac{2^{t+3}(\sqrt{3})}{2^{p+7}(\sqrt{3})\left(3+8 Z_{2}\right)}
$$


and therefore $g\left(\chi_{2}\right) \in\left(3+\delta Z_{2}\right)^{-1}=\left(3+S Z_{2}\right)$, as desired.

Lemma 3.4.4 If $g: R\left(Q_{\mathrm{\delta}}\right) \longrightarrow \mathrm{Z}_{2}^{-}$is the function of 3.1 then

$$
g\left(\chi_{1} \chi_{2}\right) \in 7+S Z_{2}
$$

Proof As in the proof of Snaith [32], Lemma 6.3.7,

$$
\left(\log _{2}\left(k\left(x_{0}\right)\right) \mid \chi_{1} \chi_{2}\right)=2^{s+1} \log _{2}\left(k\left(\rho\left(b-b^{\prime}\right)\right)\right)
$$

It follows from Corollary 2.3.8 that

$$
k\left(\rho\left(b-b^{\prime}\right)\right)=\rho\left(t_{2} /\left(\pi t_{1}\right)\right)=15+4 \sqrt{30}+2^{5} \delta
$$

for some $\delta \in Z_{2}[\sqrt{30}]$. Hence if we let $w=7+2 \sqrt{30}+2^{4} \delta$, then

$$
\begin{aligned}
\log _{2}\left(k\left(\rho\left(b-b^{\prime}\right)\right)\right) & =\log _{2}(1+2 w) \\
& =2\left(w-w^{2}+4 w^{3} / 3-2 w^{4}+\cdots\right)
\end{aligned}
$$

which lies in $2(\sqrt{30})\left(7+8 Z_{2}\right)$. Therefore, by Lemma 3.3 .2 ,

$$
\frac{\left(a_{2} \mid \chi_{1} \chi_{2}\right)}{\left(\log _{2}\left(k\left(x_{0}\right)\right) \mid \chi_{1} \chi_{2}\right)} \in \frac{2^{1+3}(\sqrt{30})}{2^{1+5}(\sqrt{30})\left(7+8 Z_{2}\right)}
$$

and therefore $g\left(\chi_{1} \chi_{2}\right) \in\left(7+8 Z_{2}\right)^{-1}=\left(7+8 Z_{2}\right)$, as desired. 
Lemma 3.4.5 If $g: R\left(Q_{8}\right) \longrightarrow \mathrm{Z}_{2}^{*}$ is the function of 3.1 then

$$
g(\nu) \in \begin{cases}3+4 Z_{2} & \text { if } \alpha^{2}=1+\sqrt{3}+\sqrt{10} / 10+\sqrt{30} / 30 \\ 1+4 Z_{2} & \text { if } \alpha^{2}=-1-\sqrt{3}-\sqrt{10} / 10-\sqrt{30} / 30\end{cases}
$$

Proof As in the proof of Snaith [32], Lemma 6.3.8,

$$
\left(\log _{2}\left(k\left(x_{0}\right)\right) \mid \nu\right)=2^{s}\left(\log _{2}\left(k\left(\left(1-x^{2}\right)\left(c_{1}-c_{2}\right)\right)\right) \mid \nu\right)
$$

If we let $D=\log _{2}\left(k\left(1-x^{2}\right)\left(c_{1}-c_{2}\right)\right)$, then by the formulae used in the proof of Lemma 3.3.2,

$$
(D \mid \nu)=\operatorname{det}\left(\begin{array}{cc}
\left(1-x^{2}-i x+i x^{3}\right)(D) & \left(x^{2} y-y-i x y-i x^{3} y\right)(D) \\
\left(y-x^{2} y-i x y-i x^{3} y\right)(D) & \left(1-x^{2}+i x-i x^{3}\right)(D)
\end{array}\right)
$$

where $i^{2}=-1$. Since $x^{2}(D)=-D$, we have

$$
\begin{aligned}
& (D \mid \nu)=\operatorname{det}\left(\begin{array}{cc}
(2-2 i x)(D) & (-2 y-2 i x y)(D) \\
(2 y-2 i x y)(D) & (2+2 i x)(D)
\end{array}\right) \\
& =4\{(D-i x(D))(D+i x(D))+(y(D)-i x y(D))(y(D)+i x y(D))\} \\
& =4\left((1+x)\left(D^{2}\right)+(y+x y)\left(D^{2}\right)\right) \\
& =4\left((1+x+y+x y)\left(D^{2}\right)\right) .
\end{aligned}
$$

The fact that $(1+x+y+x y)\left(D^{2}\right) \in 2^{5}\left(3+4 Z_{2}\right)$, which will be proved in Proposition 3.4.6, together with the results of Lemma 3.3 .2 imply that

$$
\frac{\left(a_{2} \mid \nu\right)}{\left(\log _{2}\left(k\left(x_{0}\right)\right) \mid \nu\right)} \in \frac{2^{2 t+2}\left( \pm 2^{4}\right)}{2^{s+12}\left(3+4 Z_{2}\right)}
$$


and

$$
g(\nu) \in \begin{cases}3+4 \mathrm{Z}_{2} & \text { if } \alpha^{2}=1+\sqrt{3}+\sqrt{10} / 10+\sqrt{30} / 10 \\ 1+4 \mathrm{Z}_{2} & \text { if } \alpha^{2}=-1-\sqrt{3}-\sqrt{10} / 10-\sqrt{30} / 10\end{cases}
$$

as desired.

Proposition 3.4.6 In the notation of Section 2.2.0,

$$
(1+z+y+x y)\left(\log _{2}\left(k\left(\left(1-x^{2}\right)\left(c_{1}-c_{2}\right)\right)\right)\right) \in 2^{5}\left(3+4 Z_{2}\right) .
$$

Proof As in the beginning of the proof of Corollary 2.3.6, we have

$$
k\left(\left(1-x^{2}\right)\left(c_{1}-c_{2}\right)\right)=\left(1-x^{2}\right)(x-x y)\left(t_{2}\right) \cdot\left(x^{2}-1\right)(x+y)\left(\pi l_{2} /\left(t_{2} / l_{1}\right)\right)
$$

where by Lemmas 1.3.2 and 1.3.4,

$$
t_{2}=\left(1+\xi \pi^{3}\right)\left(1+\xi \pi^{4}\right)\left(1+\xi \pi^{5}\right)\left(1+a_{6} \pi^{6}\right)\left(1+\xi \pi^{7}\right)\left(1+a_{8} \pi^{8}\right)\left(1+a_{9} \pi^{9}\right) \cdots
$$

and

$$
t_{2} / t_{1}=(1+\xi \pi)\left(\prod_{i=2}^{9}\left(1+b_{i} \pi^{i}\right)\right) \cdots
$$

The reason for choosing the terms up to $U_{L_{0}}^{9}$ is that we want to compute $k((1-$ $\left.\left.x^{2}\right)\left(c_{1}-c_{2}\right)\right)\left(\bmod \mathcal{P}_{L}^{22}\right)$. In fact we can ignore the units of $U_{L_{0}}^{8}$ in the above products. To find $k\left(\left(1-x^{2}\right)\left(c_{1}-c_{2}\right)\right) \quad\left(\bmod \mathcal{P}_{L}^{22}\right)$, we use Maple to compute the actions of 
$\left(1-x^{2}\right)(x-x y)$ and $\left(x^{2}-1\right)(x+y)$ on $\pi$ and on every term of the expressions of $t_{2}$ and $t_{2} / t_{1}$. As a result, we have, modulo $\mathcal{P}_{L}^{22}$,

$$
k\left(\left(1-x^{2}\right)\left(c_{1}-c_{2}\right)\right) \equiv 1+\pi^{8}+\pi^{13}+\pi^{16}+w \pi^{18}+w \pi^{19}+\pi^{21},
$$

where $w=0$ or 1 .

If we let $D=\log _{2}\left(k\left(\left(1-x^{2}\right)\left(c_{1}-c_{2}\right)\right)\right)$ and $\gamma=\pi^{8}+\pi^{13}+\pi^{16}+w \pi^{18}+w \pi^{19}+\pi^{21}$, then modulo $\mathcal{P}_{L}^{22}$,

$$
\begin{aligned}
D & \equiv \gamma-\gamma^{2} / 2+\gamma^{3} / 3-\gamma^{4} / 4+\cdots \\
& \equiv \pi^{12}+\pi^{13}+w \pi^{18}+w \pi^{19}+\pi^{20}
\end{aligned}
$$

Hence, modulo $\mathcal{P}_{L}^{42}$,

$$
D^{2} \equiv \pi^{24}+\pi^{26}+\pi^{33}+w \pi^{36}+\pi^{37}+(1+w) \pi^{40} .
$$

Now in the case $\alpha=\alpha_{+}$, we have, modulo $\mathcal{P}_{L}^{49}$,

$$
\begin{aligned}
(1+x+y+x y)\left(\pi^{24}\right) \equiv & \pi^{48} \\
(1+x+y+x y)\left(\pi^{26}\right) \equiv & \pi^{38}+\pi^{39}+\pi^{41}+\pi^{46}+\pi^{47}+\pi^{48} \\
(1+x+y+x y)\left(\pi^{33}\right) \equiv & \pi^{38}+\pi^{39}+\pi^{40}+\pi^{41}+\pi^{42}+\pi^{43}+ \\
& \pi^{44}+\pi^{46}+\pi^{47}+\pi^{48} \\
(1+x+y+x y)\left(w \pi^{36}\right) \equiv & 0
\end{aligned}
$$




$$
\begin{aligned}
(1+x+y+x y)\left(\pi^{37}\right) & \equiv \pi^{42}+\pi^{43}+\pi^{46}+\pi^{47}+\pi^{48} \\
(1+x+y+x y)\left((1+w) \pi^{40}\right) & \equiv 0 .
\end{aligned}
$$

In the case $\alpha=\alpha_{-}$, we also have, modulo $\mathcal{P}_{L}^{49}$,

$$
\begin{aligned}
(1+x+y+x y)\left(\pi^{24}\right) & \equiv \pi^{48} \\
(1+x+y+x y)\left(\pi^{26}\right) & \equiv \pi^{38}+\pi^{39}+\pi^{41}+\pi^{41}+\pi^{45}+\pi^{48} \\
(1+x+y+x y)\left(\pi^{33}\right) & \equiv \pi^{38}+\pi^{39}+\pi^{40}+\pi^{41}+\pi^{42}+\pi^{43}+ \\
(1+x+y+x y)\left(w \pi^{36}\right) & \equiv 0 \\
(1+x+y+x y)\left(\pi^{37}\right) & \equiv \pi^{42}+\pi^{43}+\pi^{46}+\pi^{47} \\
(1+x+y+x y)\left((1+w) \pi^{40}\right) & \equiv 0 .
\end{aligned}
$$

It now follows from the relation $\pi^{40}=2^{5}\left(1+\pi^{4}+0 \cdot \pi^{8}+\cdots\right)$ and the above identities that

$$
\begin{aligned}
(1+x+y+x y)\left(D^{2}\right) & \equiv \pi^{40}+\pi^{44}\left(\bmod \mathcal{P}_{L}^{49}\right) \\
& \equiv \pi^{40}\left(1+\pi^{4}\right)\left(\bmod \mathcal{P}_{L}^{49}\right) \\
& \equiv 2^{5}\left(1+\pi^{4}\right)\left(1+\pi^{4}\right)\left(\bmod \mathcal{P}_{L}^{49}\right) \\
& =2^{5}\left(1+\pi^{8}\right)\left(\bmod \mathcal{P}_{L}^{49}\right) \\
& \in 2^{5}\left(3+4 Z_{2}\right),
\end{aligned}
$$


as desired.

We are now in a position to state and prove the main result of this section.

Theorem 3.4.7 In the notation of Section 9.1,

$$
\left[K \operatorname{Rer}\left(k^{\prime}\right)\right]=\left\{\begin{array}{cl}
-1 & \text { if } \alpha^{2}=1+\sqrt{3}+\sqrt{10} / 10+\sqrt{30} / 10 \\
1 & \text { if } \alpha^{2}=-1-\sqrt{3}-\sqrt{10} / 10-\sqrt{30} / 10
\end{array}\right.
$$

Proof Identifying the class-group with $(\mathrm{Z} / 4)^{-}$for the moment, the arguments in the beginning of this section and the calculations of Lemma 3.4.1 to Lemma 3.4.5 show that

$$
\left[\operatorname{Ker}\left(k^{\prime}\right)\right]=[g]= \begin{cases}-(-1)^{(1 / 4) \log _{2}(\delta)} & \text { if } \alpha=\alpha_{+} \\ (-1)^{(1 / 4) \log _{2}(\delta)} & \text { if } \alpha=\alpha_{-},\end{cases}
$$

where $\delta \in 7+8 Z_{2}$ and, as usual $\alpha_{ \pm}^{2}= \pm(1+\sqrt{3}+\sqrt{10} / 10+\sqrt{30} / 10$. Since $(1 / 4) \log _{2}(7) \in 2 Z_{2}$, we have that

$$
\left[\operatorname{Ker}\left(k^{\prime}\right)\right]=\left\{\begin{array}{cc}
-1 & \text { if } \alpha=\alpha_{+} \\
1 & \text { if } \alpha=\alpha_{-}
\end{array}\right.
$$

as desired. 


\section{Bibliography}

[1] Ph. Cassou-Noguès. Quelques théorèms de base normal d'entiers, Ann. Inst. Fourier, 28,1-33,

[2] Ph. Cassou-Noguès, T. Chinburg, A. Fröhlich and M.J. Taylor. L-functions and Galois modules, London Math. Soc. 1989 Durham Symposium, L-functions and Arithmetic, Cambridge University Press, 75-139, 1989.

[3] T. Chinburg. The Analytic Theory of Multiplicative Galois Structure, Mem. A.M. Soc. 395, 1989.

[4] T. Chinburg. Exact sequences and Galois module structure, Annals Math. 121 351-376, 1985.

[5] T. Chinburg. On the Galois structure of algebraic integers and S-units Inventiones Math. 74 321-349, 1983. 
[6] T. Chinburg, M. Kolster, G. Pappas and V.P. Snaith. Quaternionic excercises in K-Theory Galois module structure, McMaster Preprint, \# 5, 1994.

[7] C.P. Curtis and I. Reiner. Methods of Representation Theary vols.I \& II, Wiley, 1984.

[8] A. Fröhlich. Classgroups and Hermitian Modules , Progress in Math. 48, Birkhäuser, 1984.

[9] A. Fröhlich. Galois Module Structure of Algebraic Integers, Ergeb. Math. 3 Folge-Band 1 Springer-Verlag, 1983.

[10] A. Fröhlich. Some problems of Galois module structure for wild extensions, Proc. London Math. Soc. 27, 193-212, 1978.

[11] A. Fröhlich. Arithmetic and Galois module structure for tame extensions, J. Reine. Angew. Math. 286/7, 380-440, 1976.

[12] A. Fröhlich. Resolvents and trace form, Math. Proc. Camb. Phil. Soc., 78, 185$210,1975$.

[13] A. Fröhlich. Artin root numbers, conductors, and representations for generalized quaternion groups, Proc. London Math. Soc., 28, 402-438, 1974. 
[14] A. Fröhlich. Artin root numbers and normal integral bases for quaternion fields, Inventiones Math., 17, 143-166, 1972.

[15] A. Fröhlich and J. Queyrut. On the functional equation for Arlin L-functions for characters of real represeniations, Inventiones Math., 20, 125-138, 1973.

[16] A. Fröhlich and M.J. Taylor. Algebraic number theory, Cambridge Studies in Advanced Math. \# 27, Cambridge University Press, 1991.

[17] C. Greither. On Chinburg's second conjecture for abelian fields, 1995 preprint.

[18] P.J. Hilton and U. Stammbach. A Course in Homological Algebra, Grad. Texts in Math., 4, Springer-Verlag, 1971.

[19] D. Holland. Additive Galois module structure and Chinöüry's invariant, J. reine angew. Math, 425, 193-218, 1992.

[20] C.U. Jensen and N. Yui. Quaternion extensions, Conf. Alg. Geom. and Comm. Alg. in honour of M. Nagata, 155-182, 1987.

[21] S. Kim A generalization of Fröhlich's theorem to wildly ramified quaternion extersions of Q, Ill. J. Math. 35, 158-189, 1991. 
[22] S. Kim. The root number class and Chinburg's second invariant, J. Alg. 153, 133-202, 1992.

[23] S. Lang. Algebra 2nd ed., Addison-Wesley, 1984.

[24] S. Lang. Algebraic Number Theory, Addison-Wesley, 1970.

[25] J. Martinet. $H_{8}$, London Math. Soc. 1975 Durham Symposium, Algebraic Number Fields (A. Fröhlich, ed.), Academic Press, 525-538, 1977.

[26] J. Martinet. Character theory and Artin L-functions, London Math. Soc. 1975 Durham Symposium, Algebraic Number Fields (A. Fröhlich, ed.), Academic Press, 1-87, 1977.

[27] J. Neukirch. Class Field Theory, Springer-Verlag, 1986.

[28] J-P. Serre. Local Fields, Grad. Texts in Math., 67, Springer-Verlag, 1979.

[29] J-P. Serre. Linear Representations of Finite Groups Grad. Texts in Math., 42, Springer-Verlag, 1977.

[30] J-P. Serre. A Course in Arithmetic, Grad. Texts in Math, 7, Springer-Verlag, 1974. 
[31] V.P. Snaith. Cyclotomic Galois module structure and the second Chinburg invariant, Math. Proc. Camb. Phil. Soc., 177, 57-, 1995.

[32] V.P. Snaith. Galois Module Structure, Fields Inst. Monographs, AMS, 1994.

[33] V.P. Snaith. Explicit Brauer Induction (with applications to algebra and number theory), Cambridge Studies in Advanced Math \# 40, Cambridge University Press, 1994.

[34] V.P. Snaith. Topological Methods in Galois Representation Theory, C.M.Soc. Monographs, Wiley, 1989.

[35] V.P. Snaith. Explicit Brauer Induction, Inventiones Math., 94, 455-478, 1988.

[36] S.H. Spanier. Algebraic Topology, McGraw-Hill, 1986.

[37] J.T. Tate. Local Constants, London Math. Soc., 1975 Durham Symposium, Algebraic Number Fields (A. Fröhlich, ed.), 89-131, Academic Press, 1977.

[38] M.J. Taylor Class Groups of Group Rings, London. Math. Soc. Lecture Notes Series, 91, Cambridge University Press, 1984.

[39] M.J. Taylor On Fröblich's conjecture for rings of integers of tame extensions, Inventiones Math., 63, 41-79, 1981. 
[40] L. Washington. Introduction to Cyclotomic Fields, Grad. Texts in Math. 83, Springer-Verlag, 1982.

[11] S.M.J Wilson. A projective invariant comparing integers in wildly ramified extensio:zs, J. reine angew. Math., 412, 35-47, 1990. 